\title{
Dexamethasone modulates immature neutrophils and interferon programming in severe COVID-19
}

\author{
Sarthak Sinha $\mathbb{1}^{1,12}$, Nicole L. Rosin $\mathbb{1}^{1,12 \bowtie}$, Rohit Arora', Elodie Labit ${ }^{\circledR 1}$, Arzina Jaffer ${ }^{1}$ ', Leslie Cao ${ }^{1}$, \\ Raquel Farias ${ }^{2,3}$, Angela P. Nguyen ${ }^{2,3}$, Luiz G. N. de Almeida ${ }^{4,5}$, Antoine Dufour ${ }^{4,5}$, Amy Bromley ${ }^{6}$, \\ Braedon McDonald ${ }^{2,3}$, Mark R. Gillrie $\mathbb{( i}^{2,7,8}$, Marvin J. Fritzler $\mathbb{D}^{2,8}$, Bryan G. Yipp ${ }^{2,3 凶}$ and \\ Jeff Biernaskie (10,9,10,11凶
}

\begin{abstract}
Although critical for host defense, innate immune cells are also pathologic drivers of acute respiratory distress syndrome (ARDS). Innate immune dynamics during Coronavirus Disease 2019 (COVID-19) ARDS, compared to ARDS from other respiratory pathogens, is unclear. Moreover, mechanisms underlying the beneficial effects of dexamethasone during severe COVID-19 remain elusive. Using single-cell RNA sequencing and plasma proteomics, we discovered that, compared to bacterial ARDS, COVID-19 was associated with expansion of distinct neutrophil states characterized by interferon (IFN) and prostaglandin signaling. Dexamethasone during severe COVID-19 affected circulating neutrophils, altered IFNactive neutrophils, downregulated interferon-stimulated genes and activated IL-1R2 ${ }^{+}$neutrophils. Dexamethasone also expanded immunosuppressive immature neutrophils and remodeled cellular interactions by changing neutrophils from information receivers into information providers. Male patients had higher proportions of IFN ${ }^{\text {active }}$ neutrophils and preferential steroid-induced immature neutrophil expansion, potentially affecting outcomes. Our single-cell atlas (see 'Data availability' section) defines COVID-19-enriched neutrophil states and molecular mechanisms of dexamethasone action to develop targeted immunotherapies for severe COVID-19.
\end{abstract}

A broad array of viral and bacterial infections can induce diffuse lung damage, ARDS, respiratory failure and death ${ }^{1-3}$. Conventionally, neutrophils are thought to be key drivers of $\mathrm{ARDS}^{4-6}$; however, neutrophil responses during severe acute respiratory syndrome coronavirus 2 (SARS-CoV-2) are still being explored. Moreover, it is unclear if lung injury and ARDS observed in COVID19 share common or distinct neutrophil responses and pathways of inflammation. Although recent studies have leveraged single-cell transcriptomics to dissect peripheral ${ }^{7-9}$ and bronchoalveolar fluid ${ }^{10-12}$ immune landscapes driving COVID-19 pathogenesis, the protocols used can inadvertently exclude polymorphonuclear granulocytes, including neutrophils, as they are sensitive cells with low RNA (and high RNase) content. In this study, like others specifically investigating neutrophil ${ }^{13,14}$, we employed whole-blood-preserving protocols that capture neutrophils (along with all other immune cell types) from critically ill patients admitted to intensive care units (ICUs) (Extended Data Fig. 1).

Relative to bacterial ARDS, COVID-19 was associated with preferential expansion of interferon (IFN ${ }^{\text {active }}$ ) and prostaglandin ( $\mathrm{PG}^{\text {active }}$ ) neutrophil states. Bacterial ARDS neutrophils had higher gene expression of anti-bacterial molecules, such as PLAC8 and CD83. Although steroids remain controversial for other forms of ARDS, dexamethasone has proven to reduce mortality in severe COVID19 (ref. $\left.{ }^{15}\right)$. In our non-randomized, pragmatic investigation, dexamethasone in severe COVID-19 affected circulating neutrophils, altered the IFNactive state, downregulated interferon-responsive genes and activated IL-1R2 ${ }^{+}$neutrophils. Dexamethasone also induced the emergence of immature neutrophils expressing ARG1 and ANXA1, genes encoding immunosuppressive molecules, which were absent in healthy controls. Additionally, dexamethasone exhibited sex-dependent effects, which might have important implications for sex-dependent outcomes and therapeutic efficacy in severe COVID-19.

\section{Results}

COVID-19 ARDS host responses in the context of bacterial ARDS. Patients with life-threatening infections requiring ICU admission receive invasive procedures, medications and intense nursing care. This includes advanced invasive or non-invasive breathing support, broad-spectrum antibiotics, sedatives, narcotics, anaesthetics, paralytics, anti-coagulants, fluids and enteral nutrition. Patients require invasive lines, including central venous and arterial catheters. These interventions make it impossible to compare life-threatening infections admitted to the ICU to mild/moderate infections (treated either on the ward or in the community) or to healthy humans. To better understand COVID-19 immune response, we compared patients with COVID-19 who were admitted to the ICU to patients with life-threatening bacterial pneumonias with ARDS who were also admitted to the ICU, to account for ICU confounders. We additionally compared these groups to healthy volunteers. ICU-admitted

\footnotetext{
'Department of Comparative Biology and Experimental Medicine, Faculty of Veterinary Medicine, University of Calgary, Calgary, AB, Canada. ${ }^{2} \mathrm{Calvin}$, Phoebe and Joan Snyder Institute for Chronic Diseases, Cumming School of Medicine, University of Calgary, Calgary, AB, Canada. ${ }^{3}$ Department of Critical Care Medicine, Cumming School of Medicine, University of Calgary, Calgary, AB, Canada. ${ }^{4}$ Department of Physiology and Pharmacology, University of Calgary, Calgary, AB, Canada. ${ }^{5} \mathrm{McC}$ aig Institute for Bone and Joint Health, University of Calgary, Calgary, AB, Canada. ${ }^{6}$ Department of Pathology and Laboratory Medicine, University of Calgary, Calgary, AB, Canada. ${ }^{7}$ Department of Microbiology, Immunology and Infectious Diseases, University of Calgary, Calgary, AB, Canada. ${ }^{8}$ Department of Medicine, Cumming School of Medicine, University of Calgary, Calgary, AB, Canada. ${ }^{9}$ Department of Surgery, Cumming School of Medicine, University of Calgary, Calgary, AB, Canada. ${ }^{10}$ Hotchkiss Brain Institute, University of Calgary, Calgary, AB, Canada. ${ }^{11}$ Alberta Children's Hospital Research Institute, University of Calgary, Calgary, AB, Canada. ${ }^{12}$ These authors contributed equally: Sarthak Sinha,

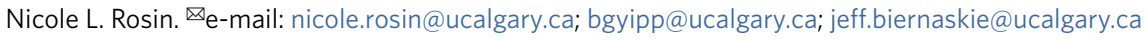


viral ARDS (for example, H1N1) would have been an interesting comparison to contextualize COVID-19-specific response; however, eradication of flu cases globally ${ }^{16}$ made it infeasible. All patients with COVID-19 were assessed for bacterial infection by culture and tested negative. All patients with COVID-19 tested positive for SARS-CoV-2 by RT-PCR. We previously confirmed an absence of viral mRNA in any circulating immune cells ${ }^{17}$. However, plasma proteomics for SARS-CoV-2-specific viral proteins detected one or more viral proteins in all COVID-19 patient serum (Extended Data Fig. 2a and Supplementary Table 1). We first compared patients with COVID-19 ARDS to bacterial sepsis (due to respiratory Staphylococcus aureus or Streptococcus pneumoniae infection) leading to ARDS, herein referred to as bacterial ARDS (Extended Data Fig. 2b). COVID-19 ARDS donors included in this comparison did not receive dexamethasone (or other immunomodulatory agents) to capture a pharmacologically unperturbed landscape (Extended Data Fig. 1 and Supplementary Table 2). We used the modified criteria for COVID-19-associated ARDS published by the World Health Organization ${ }^{18}$, which include acute onset hypoxemia and bilateral pulmonary infiltrates on X-ray without evidence of cardiac failure, with a $\mathrm{PaO}_{2} / \mathrm{FiO}_{2}$ ratio less than $300 \mathrm{mmHg}$ during mechanical ventilation or a $\mathrm{SpO}_{2} / \mathrm{FiO} 2$ ratio less than or equal to $315 \mathrm{mmHg}$ in the absence of mechanical ventilation. Our comparison included six bacterial ARDS ( $n=5$ at time point 1 (t1) and $n=4$ at time point 2 (t2)) and eight non-dexamethasone COVID-19 ARDS $(n=8$ at $\mathrm{t} 1$ and $n=4$ at $\mathrm{t} 2$ ) (Supplementary Table 2). Comparison of Sequential Organ Failure Assessment (SOFA) scores revealed no statistical difference in severity across COVID-19 ARDS versus bacterial ARDS $(P=0.17384)$, suggesting that these two cohorts comprised patients with similar disease severity. Bacterial ARDS was our comparator for COVID-19 ARDS because it was the closest control possible, as severe viral infections with ARDS were not accessible due to unusually low ICU admissions during the study period ${ }^{19}$.

Patient cohorts had similar ages, sex, days on life support and time in hospital, but patients with COVID-19 had broader racial diversity (Extended Data Fig. 2c,d and Supplementary Table 2). Bacterial ARDS induced significant neutrophilia and relative thrombocytopenia compared to near-normal circulating neutrophil numbers in COVID-19, whereas both had similar degrees of lymphopenia (Extended Data Fig. 2e). Both cohorts had similar $\mathrm{PaO}_{2} / \mathrm{FiO}_{2}$ ratios, an indicator of ARDS severity ${ }^{20}$, but patients with bacterial ARDS had significantly more kidney injury, as shown by higher serum creatinine levels (Extended Data Fig. 2f). We compared soluble inflammatory markers (Extended Data Fig. $2 \mathrm{~g}$ ) used to distinguish prototypical states, including those identified during 'cytokine storm' (Extended Data Fig. 2h) and 'cytokine release syndrome' (Extended Data Fig. 2i) ${ }^{21}$, which showed similar soluble cytokine and chemokine responses between infections. Therefore, life-threatening bacterial ARDS and COVID-19 ARDS had normal-to-elevated neutrophil counts, similar IL-6 levels and less organ failure as indicated by serum creatinine levels, all of which have been proposed as markers of COVID-19 severity ${ }^{22,23}$.
This prompted nuanced investigation into immune cell states and composition.

Our queryable atlas (see 'Data availability' section) contains single-cell RNA sequencing (scRNA-seq) data performed on whole blood at $\mathrm{t} 1$ ( $<72 \mathrm{~h}$ after ICU admission) and $\mathrm{t} 2$ ( $7 \mathrm{~d}$ after $\mathrm{t} 1)$ (Fig. 1a). Cellular identity was mapped to 30 immune cell types/ states using uniform manifold approximation and projection (UMAP) from 21 patients and 86,935 cells (Fig. 1 b and Extended Data Fig. 3a). Global magnitude of gene expression was directly compared between patients with COVID-19 and patients with bacterial ARDS (Supplementary Table 4), which revealed a more globally altered distribution of differential expression at t 1 than at $t 2$. Altered regulation of genes was most pronounced in neutrophils at $\mathrm{t} 1$, with lower neutrophil gene expression in COVID19 compared to bacterial ARDS (Fig. 1c and Extended Data Fig. $3 b, c)$. At $t 2$, the global alterations in gene expression when comparing COVID-19 to bacterial ARDS were most pronounced in plasmablasts (Fig. 1d and Extended Data Fig. 3d,e). We quantified proportions of known peripheral blood constituents, which highlighted significant differences in CD4 T cells, CD8 T cells and natural killer (NK) cells (Extended Data Fig. 3f). These data highlight substantial global differences in immune profiles between COVID-19 and bacterial ARDS.

COVID-19 drives enrichment of distinct neutrophil states. Neutrophils are a primary participant in the development of $\mathrm{ARDS}^{24}$; yet despite similar severity of ARDS between bacterial and COVID-19 cohorts, the numbers of circulating neutrophils from clinical counts were significantly different (Extended Data Fig. 2e). Global expression differences led us to hypothesize that neutrophil qualitative states might be important determinants of disease. To interrogate neutrophil dynamics, we compared pathogen-activated neutrophils in COVID-19 and bacterial ARDS to their unperturbed counterparts in healthy donors (Extended Data Fig. 4a-1). Neutrophil subclustering and integration across healthy controls, bacterial ARDS at $t 1$ and $t 2$ and COVID-19 ARDS at $t 1$ and $t 2$ revealed an absence of immature $\left(\mathrm{CD} 24^{+} \mathrm{ARG} 1^{+}\right)$and $\mathrm{IL}-1 \mathrm{R} 2^{\text {hi }}$ (IL-1R2 $2^{\text {hi }} \mathrm{CD} 163^{+}$cluster 8 and IL-1R2 $2^{\text {hi }}{ }^{2}$ TGAX ${ }^{+}$cluster 6 ) neutrophil states but expanded IL-7R $\mathrm{R}^{+}$neutrophils in healthy controls (Extended Data Fig. 4e-g). Although IFN active neutrophils were conserved across healthy controls, bacterial ARDS and COVID-19 ARDS (Extended Data Fig. 4a-g), deeper subclustering of IFN ${ }^{\text {active }}$ neutrophils revealed an emergence of discrete substates in response to COVID-19 that were not observed in either healthy controls or bacterial ARDS (Extended Data Fig. 4h-1). These substates were enriched in interferon-induced genes IFI44L and IFI44 (Extended Data Fig. 4k), molecules known to restrict respiratory viral replication $^{25}$, and exhibited an intensified type 1 IFN activation (Extended Data Fig. 4l) relative to non-COVID-19 IFN ${ }^{\text {active }}$ neutrophils.

To map pathogen-activated neutrophil dynamics with high resolution, subsequent analyses employed principal components with top-loading genes that distinguish different pathogen-activated

Fig. 1 | COVID-19 alters neutrophil maturation. a, Schematic summarizing patients with COVID-19 and bacterial ARDS profiled at t1 and t2. Comparisons presented included six bacterial ARDS ( $n=5$ at $\mathrm{t} 1$ and $n=4$ at $\mathrm{t} 2$; ${ }^{*}$ denotes that patient B3 had only the $\mathrm{t} 2$ sample pass QC and was not included at $\mathrm{t} 1$ ) and eight non-dexamethasone COVID-19 ARDS ( $n=8$ at $\mathrm{t} 1$ and $n=4$ at t2) patients who were admitted to the ICU. $\mathbf{b}$, UMAP projection of 86,935 whole blood cells from 21 patient samples, colored by Azimuth reference-mapped immune cell states. $\mathbf{c}$, $\mathbf{d}$, Kernel density estimates depicting magnitude of molecular response elicited by immune cell subsets during COVID-19 compared to bacterial ARDS at $\mathrm{t} 1$ (c) and t2 (d), calculated by summing DEG FCs for each cell state shown in a. e, UMAP plotting RNA velocity analysis of 29,653 subclustered neutrophils undergoing state transitions, colored by cluster ID. f, Stacked bar plot depicting cluster composition of clinical cohorts examined. g, UMAP colored by neutrophil clusters and overlaid with summary path curves based on vector fields and neutrophil state compositions in $\mathbf{d}$ and $\mathbf{e}$, respectively, to determine neutrophil states. h, Immunocytochemistry for S100A8/ A9 (red) and IFITM1 (green) expression on leukocyte-rich preparation from a donor with COVID-19 at t1 (representative image provided from $n=3$ replicates). i-k, Transcriptional kinetics driving expansion of IFNactive (i), bacterial ARDS-enriched (j) and PGactive (k) neutrophils. Latent time distribution of trajectory-associated Louvain clusters (left), phase portraits with equilibrium slopes of spliced-unspliced ratios (center) and RNA velocity and gene expression (right) of selected genes driving divergent maturation trajectories. Phase portraits are colored by clinical cohort. 
a

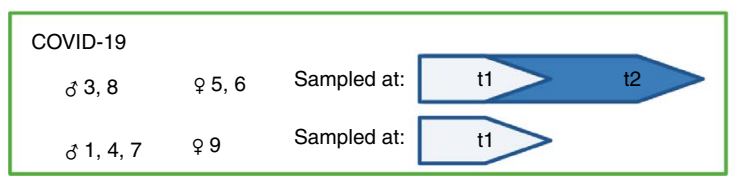

b

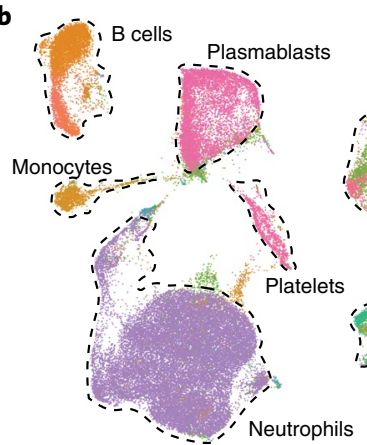

e

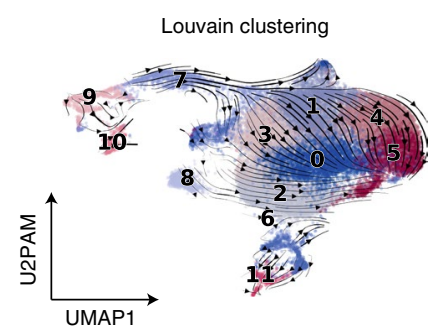

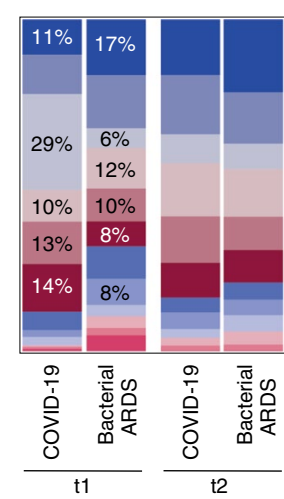

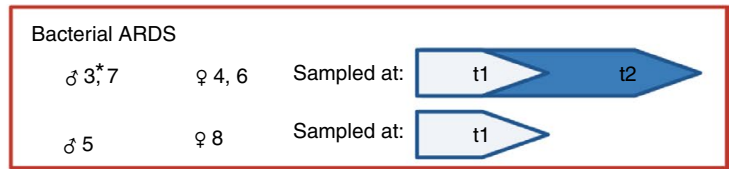

c

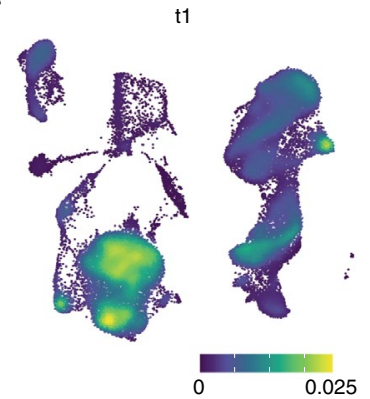

d

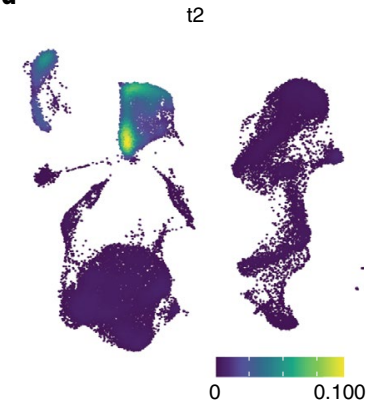

g

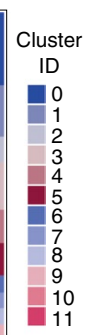

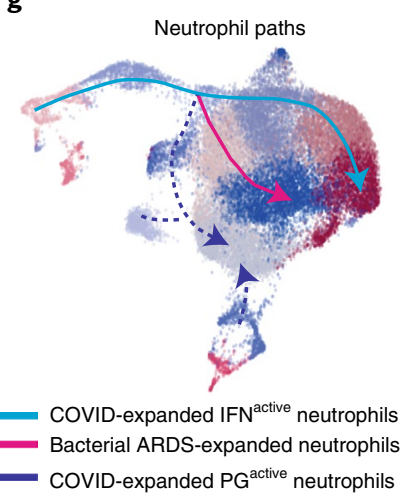

h
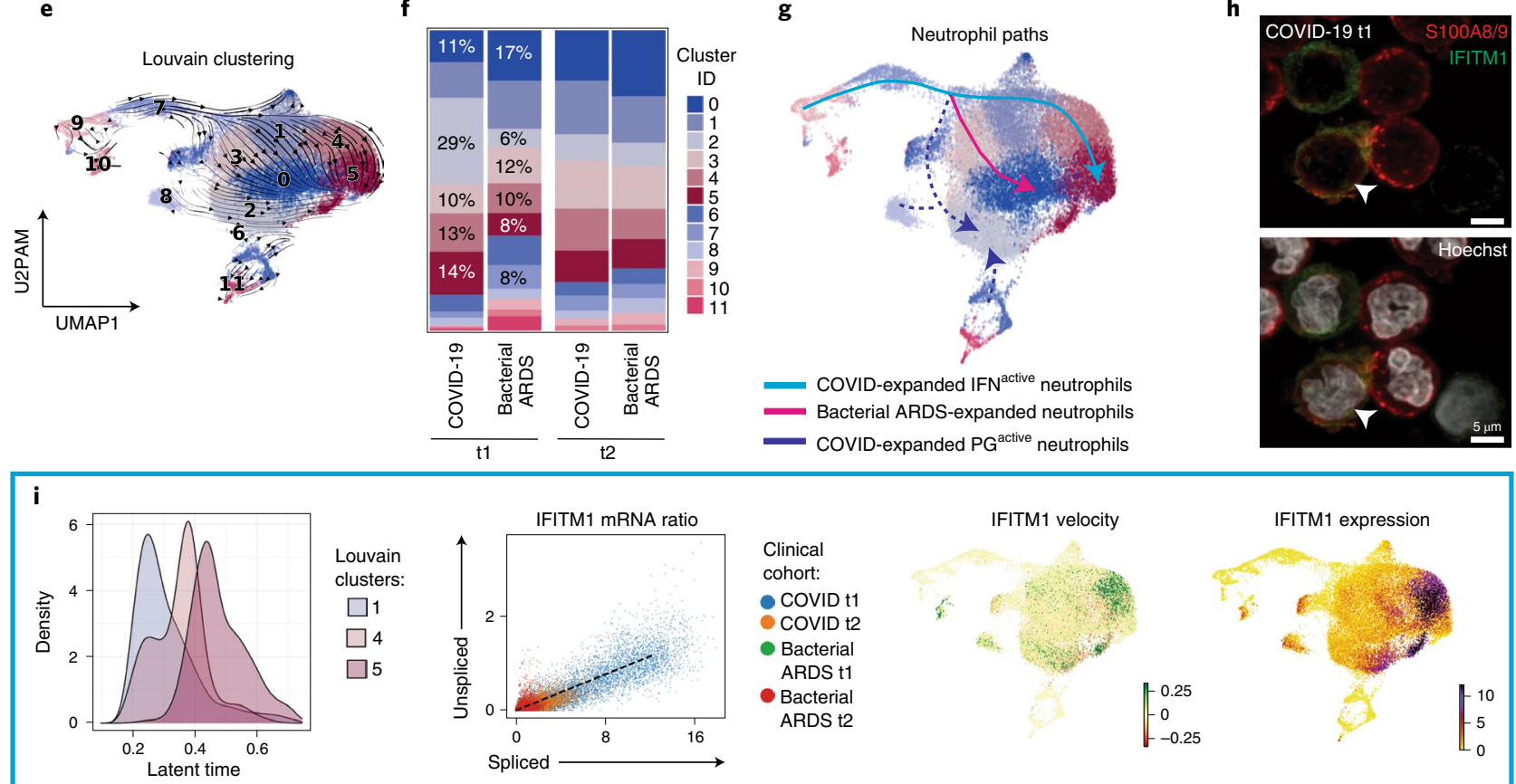
Clinical
cohort:
- COVID 11
covid 12
- Bacterial
ARDS 11
- Bacterial
ARDS t2

Spliced $\longrightarrow$
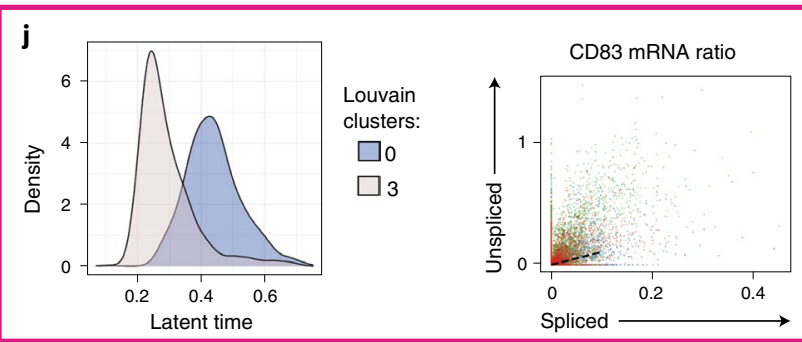

CD83 velocity

CD83 expression

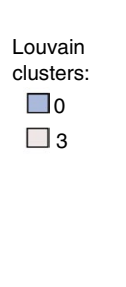

Clinical

cohort:

- COVID t1

- covid t2

- Bacterial

ARDS 11

Bacterial
ARDS $t 2$
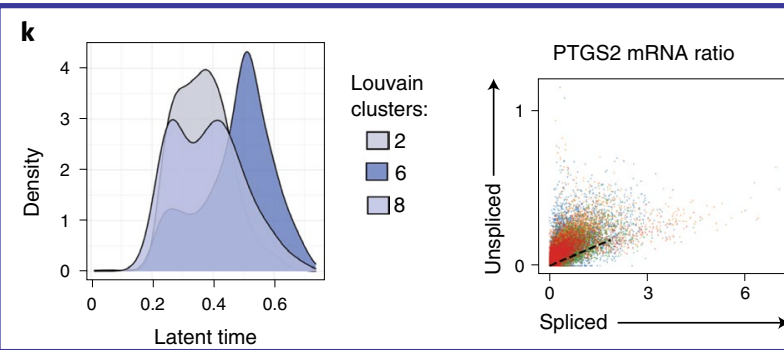

PTGS2 velocity

PTGS2 expression

Clinical
cohort:
COVID t1
COVID t2
Bacterial
ARDS t1
Bacterial
ARDS t2

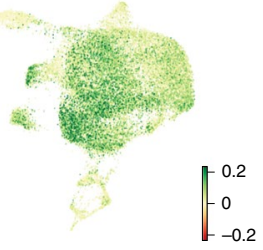

IFITM1 expression

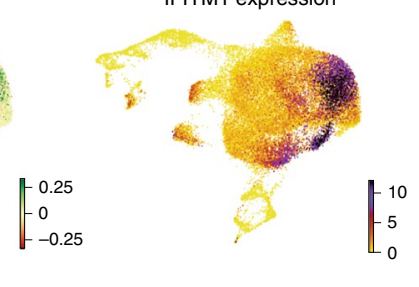


states arising during COVID-19 and bacterial ARDS (and not healthy controls) for downstream dimensionality reduction. Neutrophils were subjected to velocity analysis ${ }^{26,27}$ to reconstruct maturation dynamics. Louvain clusters (Fig. 1e), clinical cohorts, individual patients and velocity length were overlayed on velocity vector fields (Extended Data Fig. $4 \mathrm{~m}-\mathrm{q}$ ), showing three main neutrophil states. The proportions of neutrophil states were compared at $\mathrm{t} 1$, and this revealed a divergent expansion of IFN ${ }^{\text {active }}$ neutrophils (clusters 2, 4 and 5) marked by IFITM1 expression in COVID-19, which became similar to bacterial ARDS at t2 (Fig. 1f-h and Extended Data Fig. 4m-o). Gene expression of IFITM1 in neutrophils from patients with COVID-19 at t1 was confirmed by immunofluorescent staining for IFITM1 protein, co-localized with S100A8/9, and typical neutrophil nuclear morphology (Fig. 1h and Extended Data Fig. 10).

Classically, peripheral neutrophils are considered non-dividing and terminally differentiated; however, the increase in velocity length suggested the ability to alter phenotypic states once in circulation along specific paths or 'lineages'. COVID-19 neutrophils followed unique maturation paths compared to bacterial ARDS, culminating in three distinct terminal states: IFN ${ }^{\text {active }}, \mathrm{PG}^{\text {active }}$ or bacterial ARDS enriched (Fig. 1e-g and Extended Data Fig. 4m-o). The apex of this trajectory was marked by high velocity lengths, characteristic of cells undergoing differentiation (Extended Data Fig. 4p,q). COVID-19 neutrophils preferentially transitioned from the apex of the trajectory, which was an immature state (TOP2A-expressing; Extended Data Fig. 4r) to an IFN active state characterized by IFITM1, IFITM2 and IFI6 expression (clusters 1-4 and 5; Fig. 1i; Online Atlas) and activation of type I IFN signaling pathways (Extended Data Fig. 3g). Topological and geometry features of the neutrophil vector field, including identification of attractor and saddle points, were solved in an unsupervised fashion using the vector field function in Dynamo ${ }^{28}$. The continuum of neutrophil states culminating in stable IFN ${ }^{\text {active }}$ and bacterial-enriched states, as well as unstable $\mathrm{PG}^{\text {active }}$ attractor state, is shown in Supplementary Video 1. Lineage relationship was less clear for COVID-19-enriched PG active clusters defined by PG responsive genes (clusters 2, 6 and 8), with notable increases in PTGER4 and PTGS2 (or COX2), which encode a proposed target in COVID-19 (ref. ${ }^{29}$ ) (Fig. 1k and Extended Data Fig. $4 \mathrm{~s}$, $\mathrm{t}$; Online Atlas). $\mathrm{PG}^{\text {active }}$ neutrophils exhibited relative enrichment in adhesive capacity suggested by overrepresentation of cell-matrix junction pathways, such as focal adhesions mediated by TLN1, $A D A M 10, R H O B, C D 46$ and ADGRE5 (CD97), which encodes a mechanosensitive G-protein-coupled receptor (Extended Data Fig. 3h). The dominant bacterial ARDS state was characterized by expression of genes that encode anti-bacterial proteins CD83 (ref. ${ }^{30}$ ), CD177 and PLAC8 (ref. ${ }^{31}$ ) (clusters 3-0; Fig. 1j; Online Atlas). Interestingly, bacterial-enriched neutrophils were predicted to harbor ficolin-1-rich granules (Extended Data Fig. 3i). Because ficolin-1 is a recognition molecule that binds to carbohydrate structures in bacteria to initiate lectin complement pathway ${ }^{32}$, its enrichment suggests a poised state for targeting a broad range of bacterial pathogens. Together, these data showed that peripheral neutrophils have dynamic programming abilities that result in neutrophil polarization defined by emergence of IFN ${ }^{\text {active }}$ and PG $^{\text {active }}$ neutrophil states in severe COVID-19.
Unique regulatory pathways control neutrophil maturation. Rapid and robust IFN responses protect against COVID-19 severe disease, whereas delayed responses could exacerbate systemic and pulmonary inflammation ${ }^{33,34}$. Neutrophil IFN responses are not traditionally considered during infections, and neutrophils are generally considered to be homogenous, with a uniform pro-inflammatory capacity. Global neutrophil expression aligned with neutrophil state-specific markers, such as interferon response genes (IFITM1, RSAD2, IFI6 and ISG10), being more highly expressed in COVID19 neutrophils (Fig. 2a and Extended Data Fig. 4f). The inverse was the case for genes encoding anti-bacterial proteins, such as PLAC8 (Fig. 2a; Online Atlas). To interrogate infection-specific neutrophil response, we shortlisted differentially expressed features identified jointly by concordant gene and plasma protein expression changes (Fig. 2b). Interestingly, SERPINA1 (encoding protease inhibitor $\alpha-1$ antitrypsin) and PFKFB3 (encoding phosphofructokinase, a key regulator of glycolysis) were suppressed in COVID-19 neutrophils, suggesting divergence in granule-associated enzyme composition and metabolic states. Identification of differential neutrophil states prompted further exploration into factors driving neutrophil state polarization. Gene regulatory network reconstruction using single-cell regulatory network inference and clustering $(\mathrm{SCENIC})^{35}$ revealed differentially activated transcription factors (TFs) STAT1, IRF2 and PRDM1 in COVID-19 (Fig. 2c), whereas bacterial ARDS neutrophils had increased prototypical granulocyte TFs, such as CEBPA, CEBPB and STAT5B, and less defined factors such as NFE2 (Fig. 2c; Online Atlas). PRDM1 activation was most pronounced in the IFN active neutrophil population and was likely responsible for driving expression of IFN response elements (IFIT1, ISG15 and IFI6) and anti-viral signaling, such as RSAD2 and STAT1 (Fig. 2d and Supplementary Table 7; Online Atlas). A hallmark of $\mathrm{PG}^{\text {active }}$ neutrophil polarization was activation of E2F4, predicted to drive 808 genes (Fig. 2e and Supplementary Table 7), whereas neutrophil programming during bacterial ARDS included activation of STAT5B that was predicted to be upstream of ten genes (Fig. 2f and Supplementary Table 7). Consistent with the role of E2F4 as a transcriptional repressor mediating cell cycle arrest ${ }^{36}$, negative regulation of cell cycle progression was an overrepresented pathway in its SCENIC-inferred targetome (Supplementary Table 7). Interestingly, a subset of the E2F4 targetome was associated with regulating assembly of cell-matrix junctions (Supplementary Table 7), corroborating the relative enrichment in adhesive capacity seen within PG active neutrophils (Extended Data Fig. 3h). To summarize, in response to COVID-19, neutrophils were polarized by unique transcriptional regulation toward one of two main populations: either an IFN ${ }^{\text {active }}$ population or a PG ${ }^{\text {active }}$ population (Fig. $2 \mathrm{~g}$ ).

\section{Dexamethasone alters immune dynamics and plasma pro-} teomics. Conventional therapeutics have limited efficacy for COVID-19, and, although dexamethasone offers a moderate benefit, the RECOVERY trial reported that the benefit was greatest in the most severely affected patients ${ }^{15}$. Mechanisms underlying this benefit are unclear and not universal, so opportunity exists to optimize or better target this therapy. In this study, we compared eight non-dexamethasone COVID-19 ARDS ( $n=8$ at $\mathrm{t} 1$ and $n=4$ at $\mathrm{t} 2$ ) to six dexamethasone-treated COVID-19 ARDS $(n=6$ at $\mathrm{t} 1$ and

Fig. 2 | Distinct regulatory programs drive divergent neutrophil maturation. a, Consensus neutrophil DEGs upregulated (positive FC) or suppressed (negative FC) during COVID-19 in at least three of eight patients at t1 relative to bacterial ARDS. None of the patients with COVID-19 ARDS included in this comparison received dexamethasone. b. Consensus of differentially expressed features distinguishing neutrophils in COVID-19 versus bacterial ARDS jointly identified by changes in mRNA (quantified by scRNA-seq) and plasma protein (quantified by LC-MS/MS) levels. c, Differentially activated consensus TFs in neutrophils from patients with COVID-19 relative to bacterial ARDS at t1. Stacked bars depict logFC contributions of each patient with COVID-19. d-f, Gene regulatory networks preferentially driving IFNactive (PRDM1, d), PGactive (E2F4, e) and bacterial ARDS-enriched (STAT5B, f) neutrophil states. Scale bars depict kernel density estimates approximating magnitude of TF activation inferred by SCENIC-calculated AUCell scores. $\mathbf{g}$, Schematic summarizing neutrophil fates favored during COVID-19 versus bacterial ARDS (created with BioRender). 
$n=3$ at $\mathrm{t} 2$ ) patients admitted to the ICU (Supplementary Table 2). Comparison of illness severity between non-dexamethasone- versus dexamethasone-treated patients with COVID-19 ARDS using
SOFA scores obtained during ICU admission revealed no statistical difference $(P=0.33204)$, suggesting similar severity between the two groups. Median time between dexamethasone administration

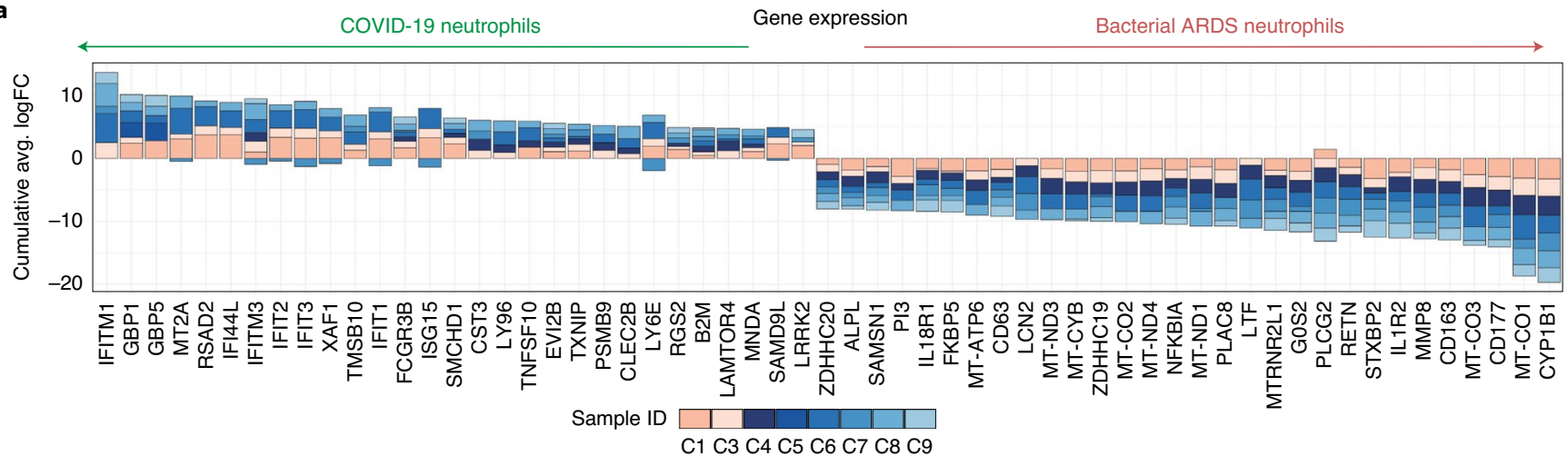

b

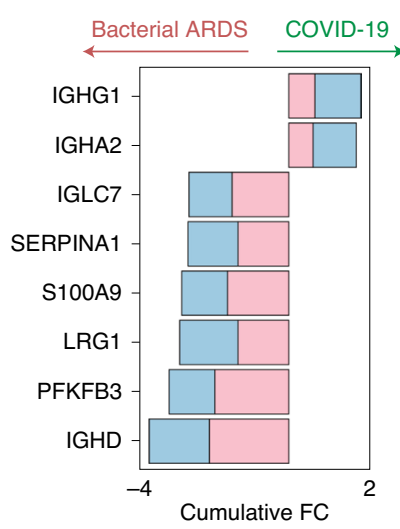

Modality $\square$ mRNA $\square$ Protein

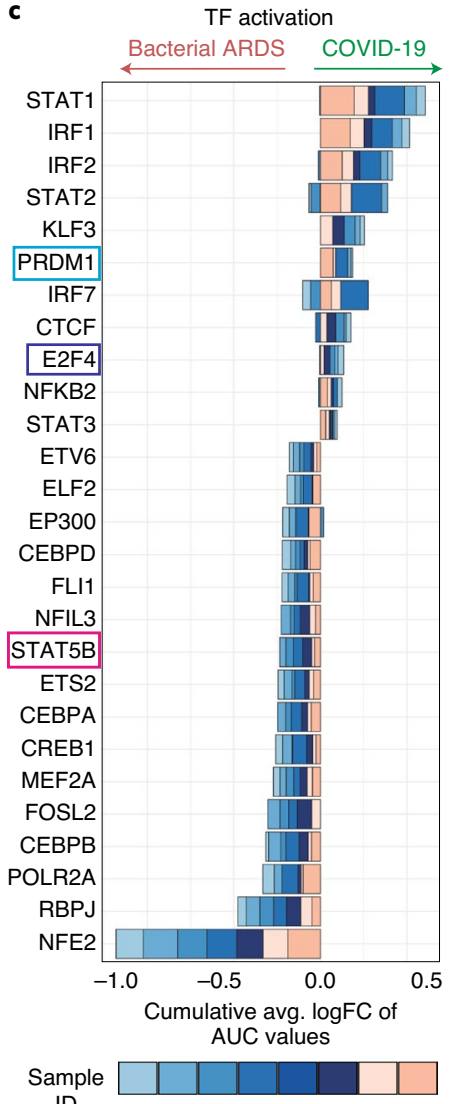

d

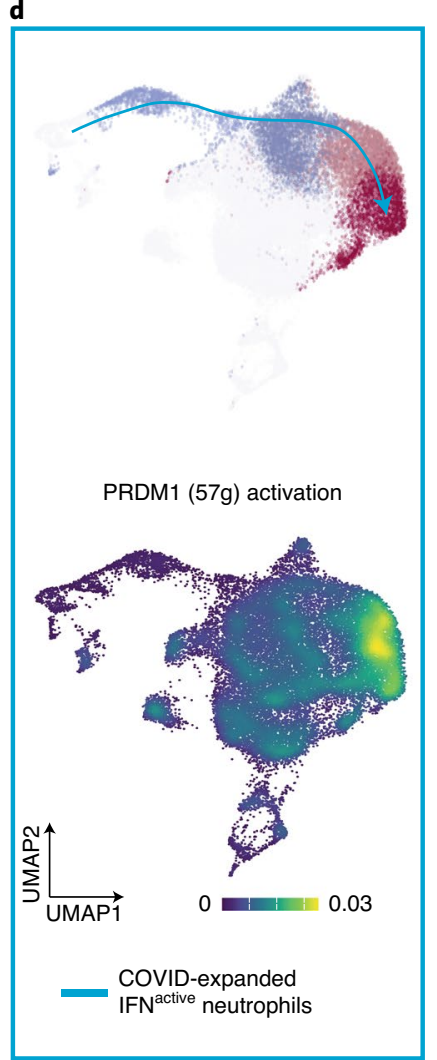

e

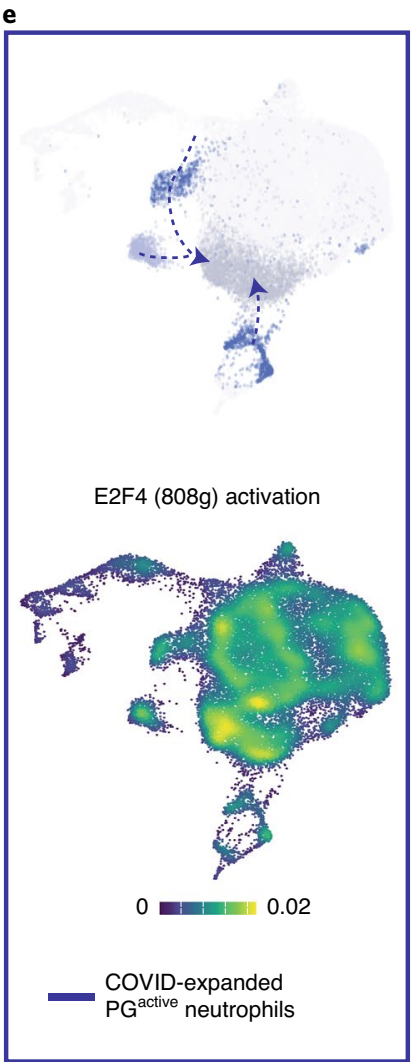

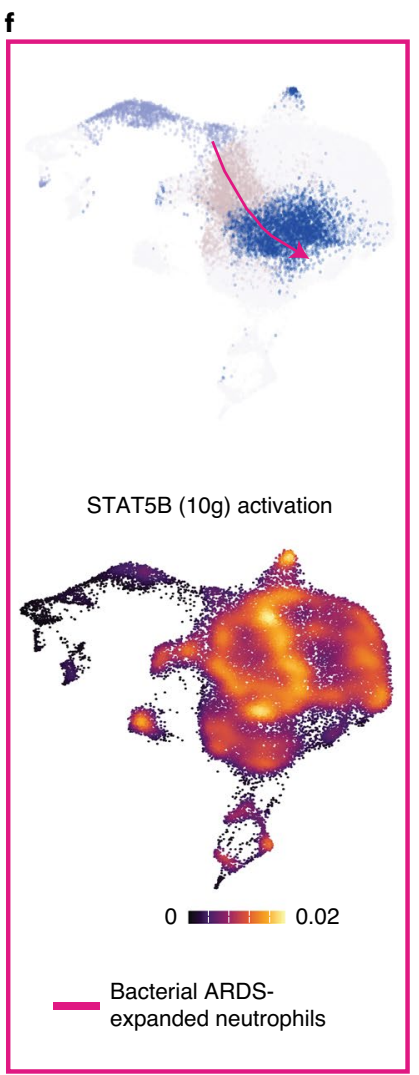

g 
to $\mathrm{t} 1 \mathrm{blood}$ draw (within $72 \mathrm{~h}$ of ICU admission) was $31 \mathrm{~h}$ (Fig. 3a, Extended Data Fig. 5a and Supplementary Table 1). Global differences in transcription were apparent at $\mathrm{t} 1$, with clear upregulation of genes in neutrophils and some T cell subsets in patients with COVID19 who were treated with dexamethasone versus those who were not treated (Fig. 3b-d, Extended Data Fig. 5b and Supplementary Table 5). At $t 1$, the dexamethasone-treated group had globally downregulated genes in naive B cells, plasmablasts and some $\mathrm{T}$ cells (Extended Data Fig. 5b-d). At t2, gene upregulation occurred in adaptive immune cells, including naive and effector CD8 T cells, with limited alterations in innate myeloid lineages, including neutrophils. Neutrophils showed clear downregulation of genes at t2, as did CD4 naive and central memory T cells (Extended Data Fig. $5 \mathrm{e}, \mathrm{f})$. Proportionally, at $\mathrm{t} 1$, dexamethasone administration was associated with an increase in cytotoxic CD4 T cells, naive B cells and plasmablasts and decreased proliferating NK cells and CD4 effector memory cells (Extended Data Fig. 5g). By t2, dexamethasone was associated with suppressed neutrophil proportions in circulation compared to untreated COVID-19 controls (13\% versus $41 \%$; Extended Data Fig. 5g). Plasma proteomics from the same cohort revealed that dexamethasone suppressed ten host proteins (S100A8, S100A9, SERPINA1, SERPINA3, ORM1, LBP, VWF, PIGR, AZGP1 and CRP) that others have identified as biomarkers distinguishing severe COVID-19 cases from mild to moderate counterparts (full host proteome queryable via Online Atlas; Supplementary Table $3)^{37-40}$. Suppression of calprotectin (S100A8/S100A9) and neutrophil serine proteases (SERPINA1 and SERPINA3) in plasma, paired with depletion of neutrophil proportions, implicates the modulation of neutrophil-related inflammatory processes as a method of action for dexamethasone.

Neutrophil IFN programs are restrained with dexamethasone. Owing to the early and sustained differences in neutrophil transcriptional programs, as well as their global depletion by t 2 with dexamethasone, more granular effects of dexamethasone on neutrophil states were evaluated. Neutrophil reclustering again identified immature neutrophils at the apex of the maturation trajectory, accelerating and exhibiting maximal divergence before $\mathrm{PG}^{\text {active }}$ and IFN ${ }^{\text {active }}$ state commitments (Fig. 3d and Extended Data Fig. 6a-e). Interestingly, we also identified $\mathrm{IL}_{-}-7 \mathrm{R}^{+}$neutrophils (comprising roughly $8 \%$ of total neutrophils) whose trajectories remained separate (Fig. 3d and Extended Data Fig. 6g,j), suggesting an entirely distinct neutrophil state. Initially, dexamethasone-treated samples had higher global transcription in $\mathrm{PG}^{\text {active }}$ neutrophils, whereas $P_{G}^{\text {active }}$ neutrophils emerged concomitant with high IL1R2 expression (IL-1R2 ${ }^{+}$) (Fig. 3e) at t2. Conversely, dexamethasone appeared to attenuate global transcription of IFN ${ }^{\text {active }}$ neutrophils at $\mathrm{t} 1$ and $\mathrm{t} 2$ (Fig. 3e,f). Remarkably, at $\mathrm{t} 1$ with dexamethasone dynamic state changes in IFN ${ }^{\text {active }}$ and IL-7R $\mathrm{R}^{+}$, neutrophils were halted, followed by preferential depletion of IFN ${ }^{\text {active }}$ subsets (Fig. $3 \mathrm{~g}$ ). Indeed, although dexamethasone was associated with a reduction in global neutrophil numbers, we also detected a reduction specifically in IFNactive neutrophils to a proportion similar to that detected in healthy controls ( $9 \%$ after dexamethasone at $\mathrm{t} 2$ versus $10 \%$ in healthy controls) (Fig. 4a and Extended Data Fig. 4a-d). Although collection of airway samples (that is, bronchoalveolar lavage fluid (BALF)) was not feasible at our institution, we leveraged two recent BALF scRNA-seq datasets ${ }^{11,41}$ to assess whether IFN ${ }^{\text {active }}$ neutrophils dominate the bronchoalveolar landscape during severe COVID-19. Projection of CSF3R $\mathrm{R}^{+} \mathrm{S} 100 \mathrm{~A} 8^{+} \mathrm{S} 100 \mathrm{~A} 9^{+} \mathrm{BALF}$ neutrophils onto our reference revealed (1) 1.5 fold change (FC) expansion of IFN ${ }^{\text {active }}$ neutrophils in severe COVID-19 relative to moderate disease (77\% versus 52\%; Extended Data Fig. 7a,b); (2) preferential activation of IFN-stimulated genes (ISGs), such as IFITM1, IFITM2, IFI6, IRF7 and ISG20, in severe COVID-19 neutrophils (Extended Data Fig. 7c); and (3) 4.7 FC higher IFN ${ }^{\text {active }}$ neutrophils in COVID-19 relative to bacterial pneumonia (14\% versus 3\%; Extended Data Fig. $7 d-f$ ). Albeit anecdotal, in our whole blood cohort, the IFN ${ }^{\text {active }}$ neutrophil state was dominant in patient S7 (ref. ${ }^{41}$ ), an 80 -year-old male with remarkably high viral titers who died from COVID-19 complications within 3-4 d of sampling (Extended Data Fig. 7f).

Consensus differentially expressed gene (DEG) analysis highlighted upregulation of $I L 1 R 2$, which encodes a decoy receptor that sequesters IL-1, and downregulation of IFITM1 as the most prominent discriminating features of dexamethasone treatment (Fig. $3 \mathrm{~h})$. Additionally, dexamethasone attenuated neutrophil expression of IFN pathways more broadly, including the reduction of IFITM1-IFITM3, IFIT1, ISG15 and RSAD2 (Fig. 3h). Examination of unspliced pre-mRNA to mature spliced mRNA ratios supported the notion that induction of immunoregulatory systems (that is, IL1R2; Fig. 3i) and suppression of IFN (that is, IFITM1) (Fig. 3j) programs were driven by differential upstream regulation of these pathways.

Dexamethasone renders neutrophils more immunosuppressive. Patients treated with dexamethasone had shifted neutrophil state compositions. Although IFN ${ }^{\text {active }}$ neutrophils were significantly depleted at $\mathrm{t} 2$, there was two-fold expansion in immature neutrophils relative to non-treated COVID-19 controls (Fig. 4a and Extended Data Figs. 6h,i and 10), which was absent in the healthy controls. Albeit circumstantial, the dominance of IFN ${ }^{\text {active }}$ neutrophils at $\mathrm{t} 1 \mathrm{in}$ the patient who died from COVID-19 in the non-dexamethasone cohort suggests the possibility that depletion of IFNactive neutrophils might be a mechanism by which dexamethasone is protective (Extended Data Fig. 8g-j). Assessment of gene regulatory networks showed that IRF7 and MEF2A exhibited opposing activation patterns, with IRF7 being the most suppressed and MEF2A being the most enhanced TFs identified with dexamethasone, which correlates with the emergence of $\mathrm{PG}^{\text {active }}$ and IL-1R2 ${ }^{+}$states and attenuation of the IFN ${ }^{a c t i v e}$ neutrophil states (Fig. $4 \mathrm{~b}$ and Extended Data Fig. 6k-

Fig. 3 | Dexamethasone suppresses IFN programs and depletes IFN ${ }^{2 a t i v e}$ neutrophils in COVID-19. a, Schematic summarizing patients with COVID-19 who were treated with or without dexamethasone profiled at $\mathrm{t} 1$ and $\mathrm{t} 2$. Comparisons presented included eight non-dexamethasone-treated COVID-19 ARDS ( $n=8$ at $\mathrm{t} 1$ and $n=4$ at t2) and six dexamethasone-treated COVID-19 ARDS ( $n=6$ at t 1 and $n=3$ at t2) patients who were admitted to the ICU. b, UMAP projection of 80,994 whole blood cells from 21 patient samples, colored by Azimuth reference-mapped immune cell states. $\mathbf{c}$, $\mathbf{d}$, Kernel density estimates depicting magnitude of molecular response elicited by immune cell subsets after dexamethasone treatment at $\mathrm{t} 1$ (c) and $\mathrm{t} 2$ (d), calculated by summing DEG FCs for each cell state shown in a. e, Neutrophil states overlaid on a UMAP of 23,193 subclustered neutrophils from dexamethasone- and non-dexamethasone-treated patients with COVID-19, colored by cluster ID. f, Magnitude of molecular response elicited by each neutrophil state after dexamethasone treatment calculated by summing DEG FCs for each cell state shown in $\mathbf{d}, \mathbf{g}$, RNA velocity vector length (indicating rate of differentiation/ state transition) in dexamethasone- and non-dexamethasone-treated neutrophils at $\mathrm{t} 1$ and $\mathrm{t} 2$. $\mathbf{h}$. Consensus neutrophil DEGs upregulated (positive FC) or suppressed (negative FC) after dexamethasone in at least three of six patients with COVID-19 at t1 relative to non-dexamethasone COVID-19 controls. Stacked bars depict logFC contribution of each dexamethasone-treated patient. i, j, Differential splicing kinetics drives activation of IL-1R2 (i) and suppression of IFITM1 expression ( $\mathbf{j}$ ) after dexamethasone treatment. Phase portraits show equilibrium slopes of spliced-unspliced mRNA ratios. Green denotes most upregulated and red denotes most downregulated DEGs with COVID-19 (f). HSPC, hematopoietic stem and progenitor cell. Dex, dexamethasone. 
$\mathrm{m})$. To assess the generalizability of the dexamethasone-regulated DEGs identified in our cohort, we asked whether they accurately predicted mortality due to COVID-19 in a larger validation cohort. By leveraging a whole blood bulk RNA-seq dataset from 103 patients with COVID-19 (refs. ${ }^{42,43}$ ), we scored each sample by the aggregated expression of dexamethasone-suppressed DEGs at t1 and $\mathrm{t} 2$ (Supplementary Table 5). Interestingly, suppressed DEGs at t2 (but not t1) proved to be a far superior predictor of 28-d mortality (area under the curve $(\mathrm{AUC})=0.78$; confidence interval (CI), 0.67-0.89) compared to clinical severity scales, such as SOFA

a

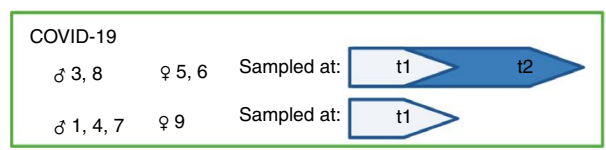

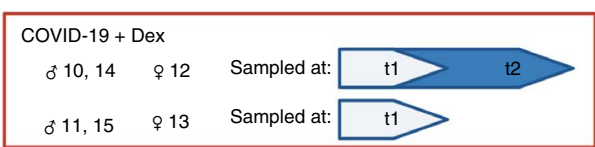

b

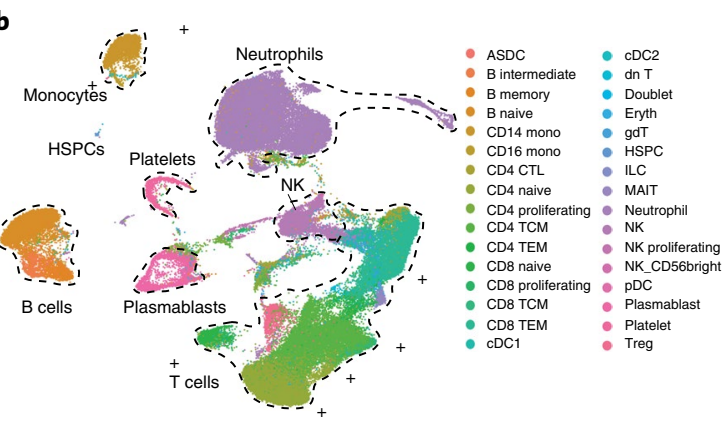

C

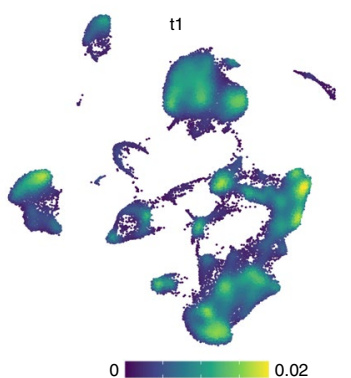

d

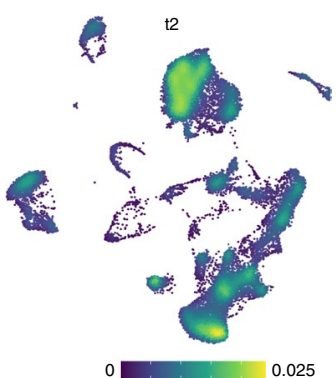

e
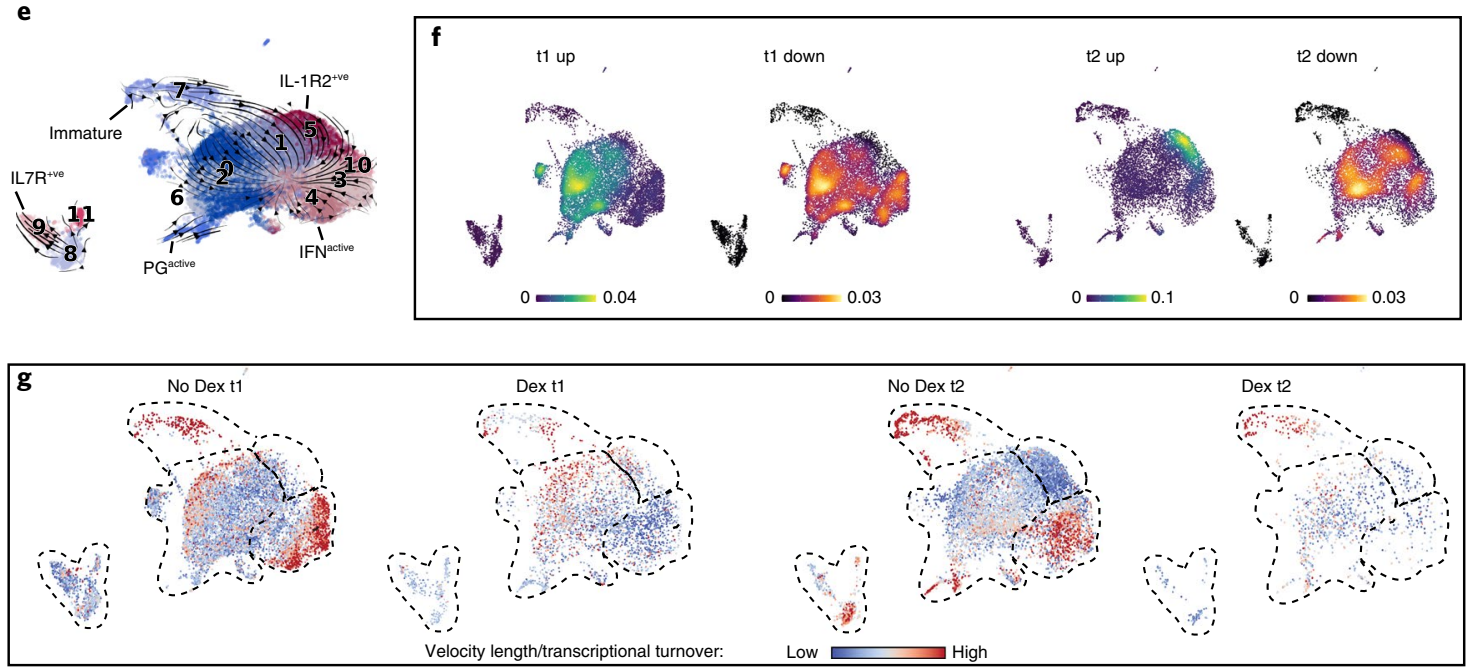

h
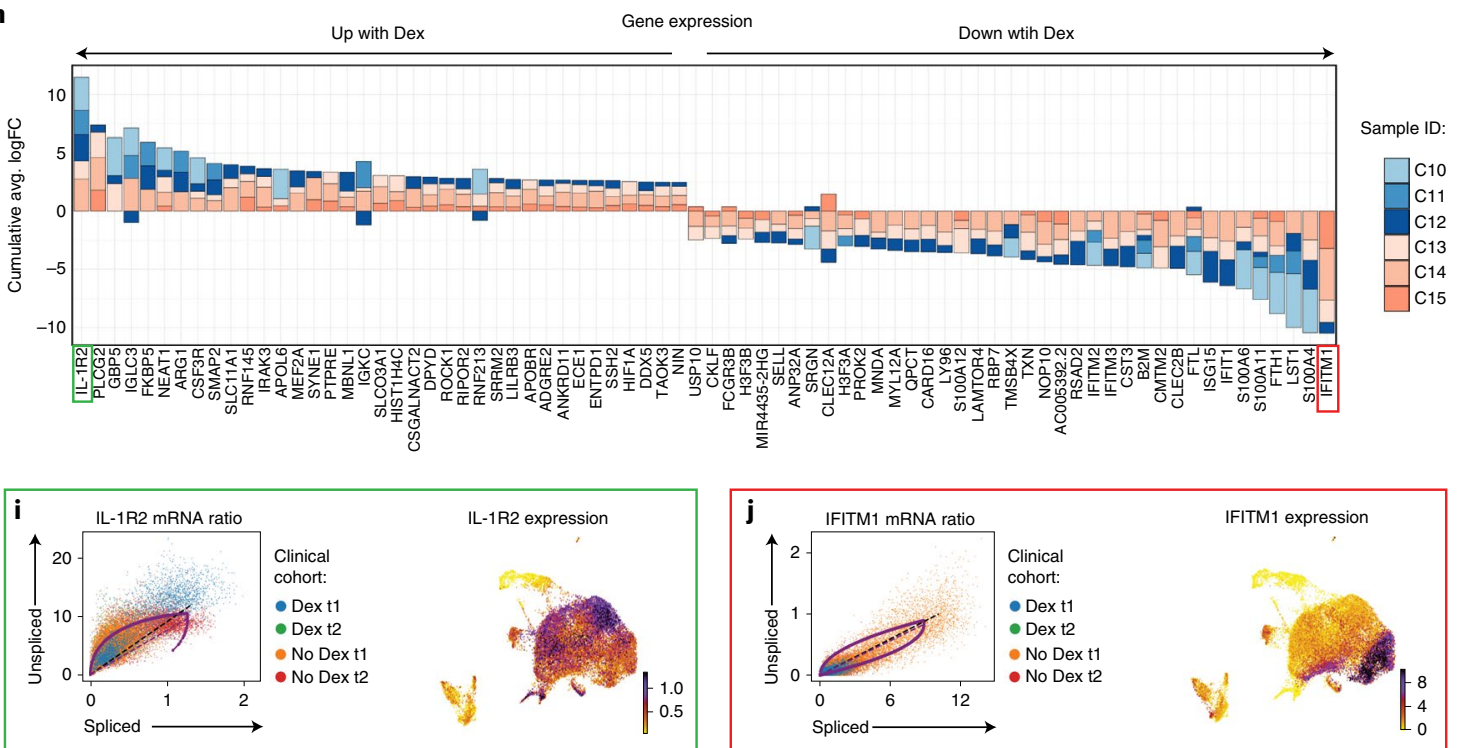
$(\mathrm{AUC}=0.67 ; \mathrm{CI}, 0.51-0.82)$ across all classification thresholds (Fig. 4c). Multi-modal (transcriptional and plasma proteomic) assessments corroborated suppression of mature neutrophil programs (for example, $\beta$-2-microglobulin encoded by $B 2 M$; Online Atlas) along with concomitant activation of IFN-restraining cytokines (for example, IK cytokine, a potent inhibitor of IFN- $\gamma$ ) after dexamethasone (Fig. 4d). Unexpectedly, steroid administration was associated with an increase in circulating immature neutrophils, which highly expressed TOP2A, and activated ATF4 and JDP2, TFs seen in undifferentiated cells or those undergoing nuclear reprogramming (Extended Data Fig. 6h). Because TOP2A marks proliferating cells $^{44}$, we asked whether dexamethasone increased proliferation of immature neutrophils to drive their expansion. There was no change in frequency of TOP $2 \mathrm{~A}^{+}$immature neutrophil after dexamethasone treatment $\left(8 \%\right.$ TOP $2 \mathrm{~A}^{+}$in dexamethasone-treated versus $10 \%$ in non-dexamethasone-treated across $\mathrm{t} 1$ and $\mathrm{t} 2 ; \chi^{2}=4.58, P=0.21$ ), suggesting that dexamethasone does not stimulate division of circulating (immature) neutrophils. Immature neutrophils expressed high levels of ARG1, ANXA1 (Fig. 4e) and CD24 (both mRNA and protein; Extended Data Fig. 6i), suggesting that additional immunomodulatory function ${ }^{45-49}$ expanded with dexamethasone. Both $A R G 1$ and ANXA1 express glucocorticoid response elements, emphasizing the possibility of their direct regulation by dexamethasone treatment ${ }^{50,51}$.

To further understand the role of neutrophils during COVID19 and the effects of dexamethasone, we investigated cellular connectomes. Cellular interactions between many cell types (including highly interactive neutrophils) were noted (Extended Data Fig. 8a), and dexamethasone altered the globally predicted interactions by suppressing both number and strength of intercellular interactions (Extended Data Fig. 8b,c). Dexamethasone enhanced and suppressed (Extended Data Fig. 8d) several unique neutrophil-driven signaling networks. Annexin signaling, which was enhanced in the immature neutrophils and which are powerful glucocorticoid targets for resolving inflammation ${ }^{52}$, was augmented between neutrophils and the other circulating immune cells when patients received dexamethasone (Fig. 4f,g). Of note is the direction of annexin family signaling, which switched from incoming toward neutrophils to being outgoing from neutrophils toward B intermediate and memory cells and MAIT cells after dexamethasone (Fig. 4f,g and Extended Data Fig. 8e,f). Re-patterning in annexin signaling was primarily driven by a 2.4 -fold expansion of immature neutrophils after dexamethasone and not due to a change in ANXA1 expression (12.4 versus 12.0 log-normalized unique molecular identifier (UMI) in non-treated and treated, respectively) in immature subsets across non-dexamethasone-treated and dexamethasone-treated donors. Dexamethasone altered neutrophil states by promoting expansion of an $\mathrm{ARG}^{+} \mathrm{ANXA1}^{+}$immature state with immunosuppressive features and altered global communication structure such that neutrophils became active instructors of peripheral immune cells.

Neutrophil response to dexamethasone is sexually dimorphic. Given that the clinical benefit of dexamethasone is more evident in males $^{15}$, and because males are predisposed to more severe COVID-19 presentations and outcomes ${ }^{53}$, we surmised that dexamethasone incites sexually dimorphic effects. Our retrospective province-wide audit comparing 72 pre-dexamethasone (51 male and 21 female) versus 1,581 post-dexamethasone (1,013 male and 568 female) ICU-admitted patients confirmed a preferential mortality benefit in male patients with COVID-19 (Extended Data Fig. 9a,b). Dexamethasone-treated patients had 525 neutrophil DEGs across both sexes, whereas 892 were uniquely modulated in either males or females (Supplementary Table 6). Of the jointly modulated DEGs, a subset (24 of 525) exhibited statistically significant dimorphism in magnitude or direction of regulation (Extended Data Fig. 9c,d). Although neutrophils were depleted in both sexes with dexamethasone, this was particularly pronounced in males (1.9 FC higher at $\mathrm{t} 1$ and 3.4 FC higher at t2; Extended Data Fig. 9e). Of the two salient neutrophil state alterations, an immature (ARG1 ${ }^{+}$immunosuppressive) state was preferentially expanded with dexamethasone in males (Extended Data Fig. 9e), whereas ISGs were preferentially suppressed (Extended Data Fig. 9f) and IFN ${ }^{\text {active }}$ states were depleted in females (Extended Data Fig. 9g,h) at t1 and t2 (Fig. 4h,i). Sexually dimorphic dexamethasone effects on neutrophil maturation kinetics might, in part, explain these alterations. Dynamo-inferred vector fields (predictions of neutrophils' near-future states) revealed dexamethasone-induced features that were preferentially regulated in females. Dexamethasone was associated with accelerated immature $\left(\mathrm{ARG}^{+}{ }^{+}\right.$immunosuppressive) neutrophil differentiation at $\mathrm{t} 1$ and stunted IFNactive neutrophil transitions at $\mathrm{t} 2$ (Extended Data Fig. 9i,j). This suggests that the sexually dimorphic effect of dexamethasone might be due to dimorphic alterations of neutrophil maturation, resulting in preferential depletion of IFN ${ }^{\text {active }}$ neutrophils concomitant with a lack of immature neutrophil expansion in females.

\section{Discussion}

Surviving SARS-CoV-2 depends on striking a temporal balance between inciting viral clearance immune programs during the early stage and subsequently restraining those same programs at later stages to limit immunity-induced damage. IFN signaling stands at the nexus between anti-viral immunity and overactive effector immune programs that inadvertently compromise tissue function and threaten survival ${ }^{54}$. Our work uncovered a stable neutrophil state with signature downstream IFN signaling that is selectively expanded during late-stage COVID-19 infection. Inborn errors ${ }^{34}$ and suppressed early-stage ${ }^{6}$ IFN signaling predict COVID-19 severity, and increased IFN ${ }^{a c t i v e}$ neutrophils in females correlated with decreased mortality ${ }^{55}$. Thus, early initiation of IFN therapy has been suggested to mitigate disease severity ${ }^{56,57}$. Given these observations, one might posit that IFN activity in neutrophils represents a concerted host anti-viral program.

Interestingly, immunosuppression with dexamethasone, a corticosteroid known to improve mortality in patients hospitalized with COVID-19 (ref. ${ }^{15}$ ), was associated with global alteration of neutrophils as well as suppression of neutrophilic IFN networks and preferential depletion of COVID-19-enriched IFN ${ }^{\text {active }}$ neutrophils. These altered neutrophil states shared striking resemblances to

Fig. 4 | Dexamethasone expands immunosuppressive neutrophils and repatterns their interactions in COVID-19. a, Neutrophil states mapped onto Louvain-clustered UMAP, with comparison of neutrophil composition between dexamethasone- and non-dexamethasone-treated samples at $\mathrm{t} 1$ and $\mathrm{t} 2$. b. Consensus TFs activated or suppressed after dexamethasone in at least three of six patients at $\mathrm{t} 1$ and predicted activity of MEF2A and IRF7, two of the most differentially regulated TFs, after dexamethasone. $\mathbf{c}$. ROC curves assessing the discriminatory capacity of dexamethasone-suppressed DEGs at $\mathrm{t} 1$ and $\mathrm{t} 2$ and SOFA scores for predicting 28-d mortality in a validation cohort of 103 bulk whole blood RNA-seq samples where 17 cases were fatal. d, Consensus of differentially expressed neutrophil features upregulated (positive FC) or suppressed (negative FC) after dexamethasone jointly identified by changes in mRNA (quantified by scRNA-seq) and plasma protein (quantified by LC-MS/MS) levels. e, Immature and IL-1R2 ${ }^{+}$neutrophil subsets express high levels of immunosuppressive neutrophil markers ARG1 and ANXA1. $\mathbf{f}, \mathbf{g}$, Topology of annexin signaling family without (f) and with (g) dexamethasone treatment (edges filtered to those where neutrophils function as senders or recipients of annexin signals). $\mathbf{h}$, Neutrophil state composition separated by sex and dexamethasone status at $\mathrm{t} 1$ and $\mathrm{t} 2$. i, Schematic summarizing the effects of dexamethasone on neutrophil fates and function in COVID-19 after dexamethasone treatment (created with BioRender). Dex, dexamethasone. 
bacterial ARDS, suggesting that installation of generalized microbicidal programs ameliorate the overzealous neutrophil responses during COVID-19 (and perhaps during other viral infections).
Although neutrophil ISG activation might promote anti-viral immunity during early stages of SARS-CoV-2 infection, sustained IFN activation during late stages (for example, patients admitted a

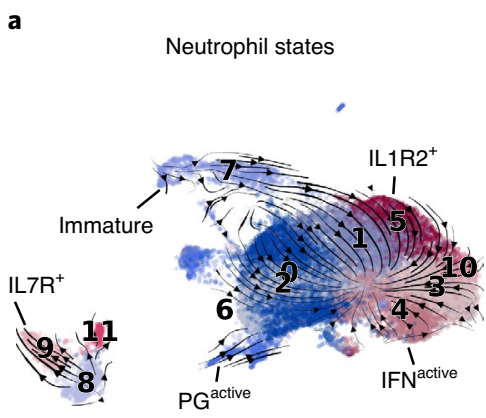

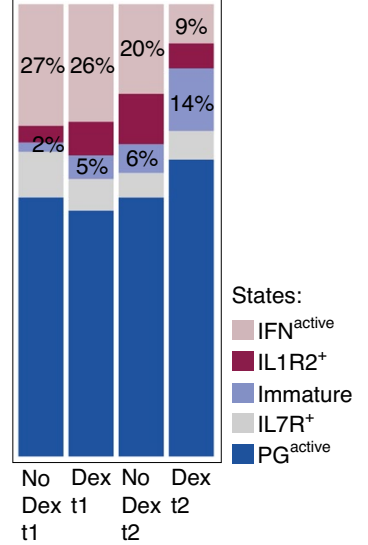

c

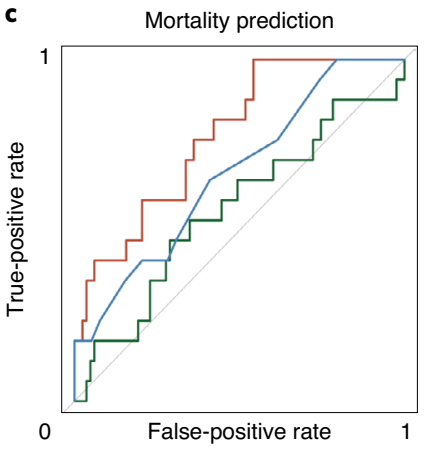

- 7-d Dex-suppressed (AUC: 0.777)

- SOFA (AUC: 0.666)

- 72-h Dex-suppressed (AUC: 0.560)

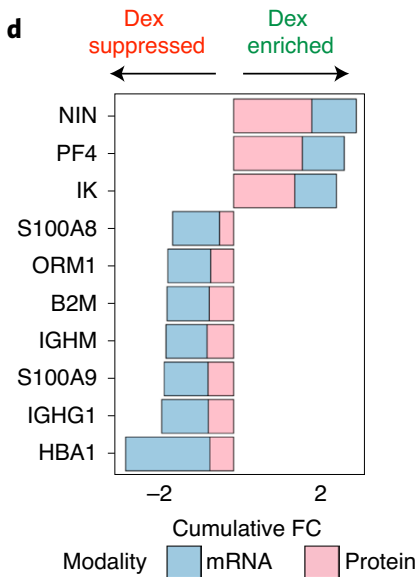

h

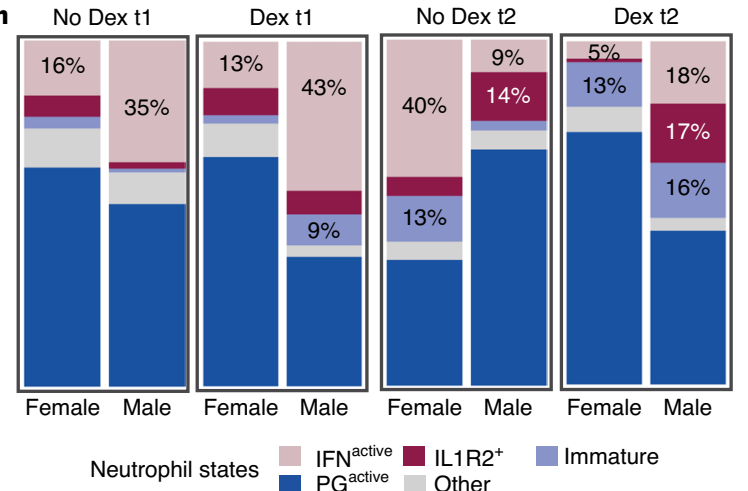

b

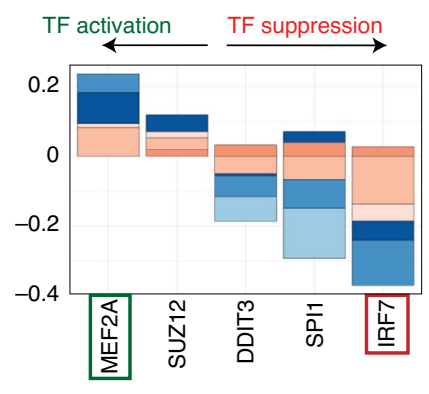

Sample ID:

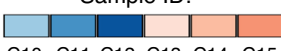

C10 C11 C12 C13 C14 C15
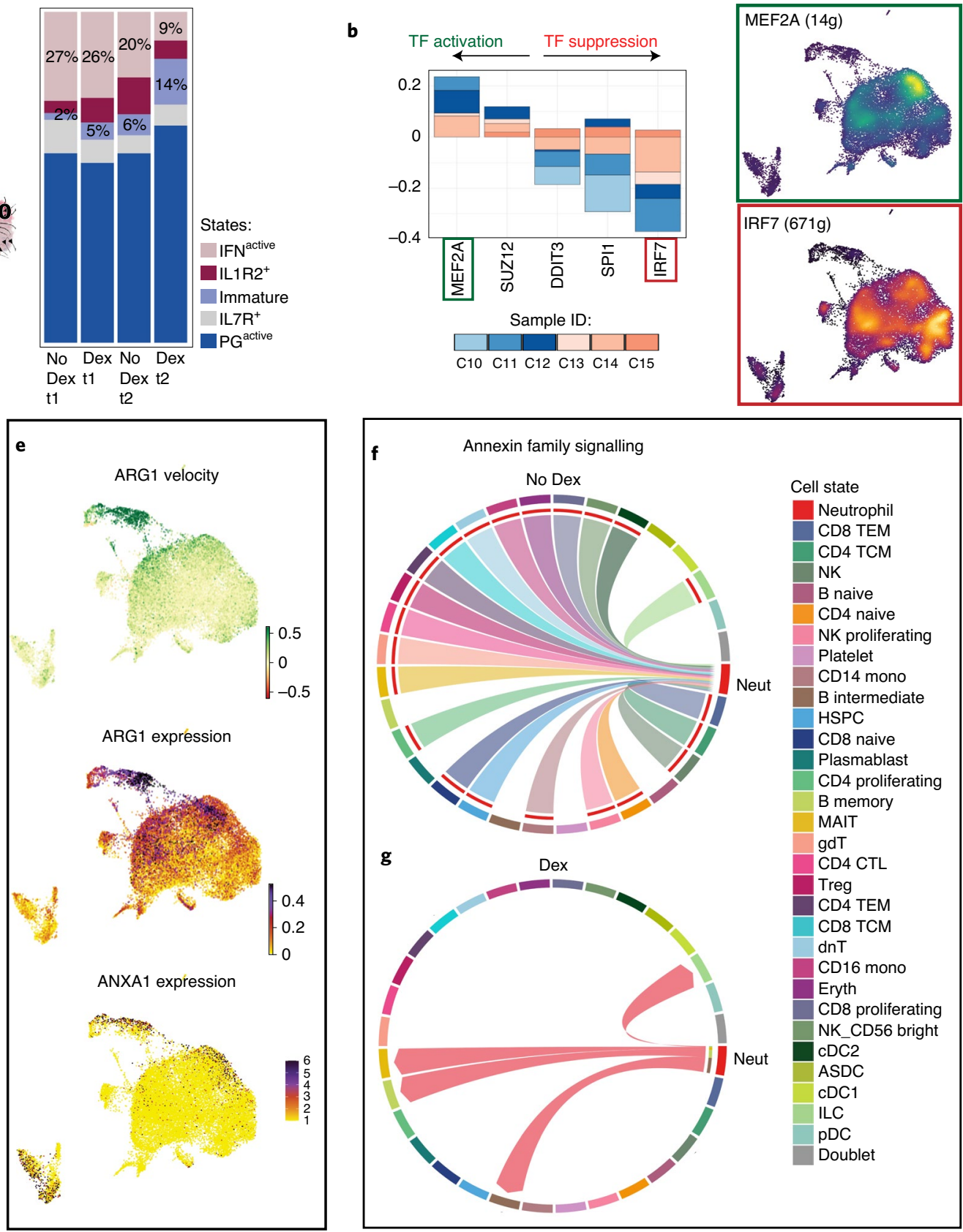

Cell state

Neutrophil

CD8 TEM

CD4 TCM

NK

$B$ naive

CD4 naive

NK proliferating

Platelet

CD14 mono

$B$ intermediate

HSPC

CD8 naive

Plasmablast

CD4 proliferating

B memory

MAIT

gdT

CD4 CTL

Treg

CD4 TEM

CD8 TCM

$\mathrm{dnT}$

CD16 mono

Eryth

CD8 proliferating

NK_CD56 bright

cDC2

ASDC

CDC1

ILC

$\mathrm{pDC}$

Doublet i

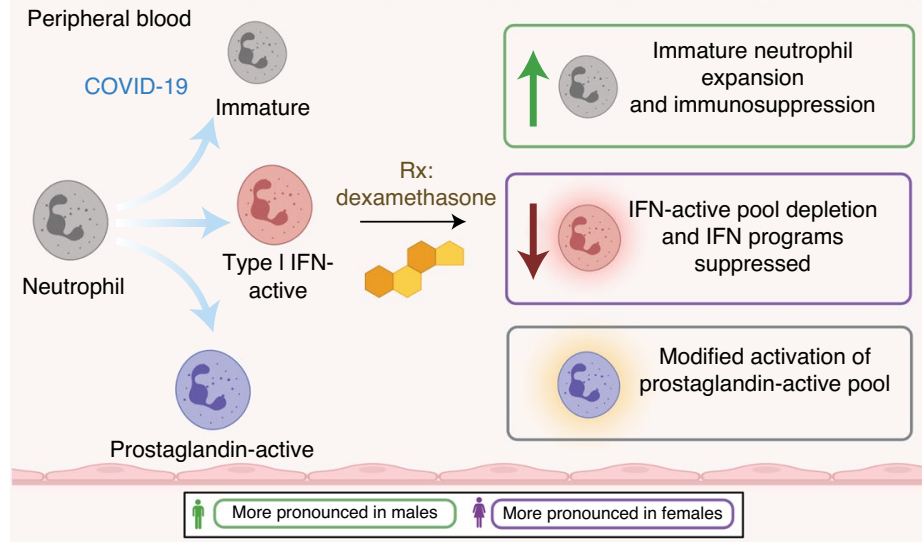


to the ICU with severe disease) could drive immunopathology of COVID-19. Indeed, positive correlation between neutrophil type 1 IFN programs and COVID-19 severity ${ }^{7,58}$, paired with our observation that IFN ${ }^{\text {active }}$ neutrophils dominate the bronchoalveolar microenvironment during severe COVID-19 (ref. ${ }^{11}$ ), support this view.

Another neutrophil state that emerged with COVID-19 (and was absent in healthy controls) was an $\mathrm{ARG1}^{+}$immature and immunosuppressive state with immunomodulatory properties ${ }^{45-49}$. This state was significantly expanded with dexamethasone, suggesting a second route of effect of dexamethasone on both neutrophils and systemic innate immune response. Whether immature neutrophils arise due to enhanced liberation in bone marrow, release of marginated cells or restrained differentiation due to dexamethasone remains to be determined. Although dexamethasone did not appear to increase the frequency of proliferating immature neutrophils, future experiments should interrogate dexamethasone-induced expansion of granulocyte/macrophage progenitors within the bone marrow or hastened liberation of immature neutrophils to explain the expanded pool of immature neutrophils in circulation. Further investigation into direct versus indirect effects of dexamethasone on neutrophils would impart insights into dexamethasone autonomous effects. Immunotherapies supporting innate anti-viral immunity by decoupling IFN-exaggerated neutrophil response while reinforcing suppressor states might limit neutrophil pathogenicity and provide benefit for severe COVID-19.

Our study has three major limitations. First, it is a pragmatic retrospective cohort study and not a randomized controlled trial. During the study enrollment period, dexamethasone became standard of care, leading to a fixed size and sex of the non-dexamethasone group. Non-random allocation and small sample size might inadvertently introduce selection bias and limit generalizability of dexamethasone findings. Second, comparisons against bacterial ARDS, and not another respiratory viral infection, preclude assessment of whether dynamics defined are specific to SARS-CoV-2. Finally, a subset of patients sampled at $\mathrm{t} 1 \mathrm{\text {was}}$ discharged from the ICU before $\mathrm{t} 2$ collection, precluding estimation of temporal changes.

Several exciting avenues of study remain, including investigating where neutrophil polarization occurs in response to both dexamethasone and COVID-19 infection. Given that pre-neutrophils in marrow become non-mitotic and can enter the bloodstream in an early or immature form (morphologically defined as band cells), we speculate that neutrophil state alterations occur after they enter circulation; however, this needs formal testing. Because neutrophils do not divide, we think it is unlikely that the increase in polarized subsets is due to expansion or replication of pre-existing polarized states that were observed at low numbers in healthy controls. However, there are no definitive data to know if polarized neutrophils arise from distinct lineage-restricted precursor pools in the bone marrow. Defining mechanisms that drive neutrophil state polarization will shed light on whether neutrophil state changes reflect a dynamic continuum or are the result of pre-ordained functional programming and will enable researchers to therapeutically target unwanted neutrophil states or enhance beneficial neutrophil states to combat disease.

\section{Online content}

Any methods, additional references, Nature Research reporting summaries, source data, extended data, supplementary information, acknowledgements, peer review information; details of author contributions and competing interests; and statements of data and code availability are available at https://doi.org/10.1038/ s41591-021-01576-3.

Received: 4 August 2021; Accepted: 12 October 2021; Published online: 15 November 2021

\section{References}

1. Matthay, M. A. et al. Acute respiratory distress syndrome. Nat. Rev. Dis. Prim. 5, 18 (2019).

2. Wang, D. et al. Clinical characteristics of 138 hospitalized patients with 2019 novel coronavirus-infected pneumonia in Wuhan, China. JAMA 323, 1061-1069 (2020).

3. Zhu, N. et al. A novel coronavirus from patients with pneumonia in China, 2019. N. Engl. J. Med. 382, 727-733 (2020).

4. Middleton, E. A. et al. Neutrophil extracellular traps contribute to immunothrombosis in COVID-19 acute respiratory distress syndrome. Blood 136, 1169-1179 (2020).

5. Veras, F. P. et al. SARS-CoV-2-triggered neutrophil extracellular traps mediate COVID-19 pathology. J. Exp. Med. 217, e20201129 (2020).

6. Combes, A. J. et al. Global absence and targeting of protective immune states in severe COVID-19. Nature https://doi.org/10.21203/rs.3.rs-97042/v1 (2021).

7. Wilk, A. J. et al. A single-cell atlas of the peripheral immune response in patients with severe COVID-19. Nat. Med. 26, 1070-1076 (2020).

8. Xie, X. et al. Single-cell transcriptome profiling reveals neutrophil heterogeneity in homeostasis and infection. Nat. Immunol. 21, 1119-1133 (2020)

9. Lee, J. S. et al. Immunophenotyping of COVID-19 and influenza highlights the role of type I interferons in development of severe COVID-19. Sci. Immunol. 5, eabd1554 (2020).

10. $\mathrm{Xu}, \mathrm{G}$. et al. The differential immune responses to COVID-19 in peripheral and lung revealed by single-cell RNA sequencing. Cell Discov. 6, 73 (2020).

11. Liao, M. et al. Single-cell landscape of bronchoalveolar immune cells in patients with COVID-19. Nat. Med. 26, 842-844 (2020).

12. Wauters, E. et al. Discriminating mild from critical COVID-19 by innate and adaptive immune single-cell profiling of bronchoalveolar lavages. Cell Res. 31, 272-290 (2021).

13. Silvin, A. et al. Elevated calprotectin and abnormal myeloid cell subsets discriminate severe from mild COVID-19. Cell 182, 1401-1418 (2020).

14. Loske, J. et al. Pre-activated antiviral innate immunity in the upper airways controls early SARS-CoV-2 infection in children. Nat. Biotechnol. https://doi. org/10.1038/S41587-021-01037-9 (2021).

15. RECOVERY Collaborative Group et al. Dexamethasone in hospitalized patients with Covid-19. N. Engl. J. Med. 384, 693-704 (2021).

16. Jones, N. How COVID-19 is changing the cold and flu season. Nature 588, 388-390 (2020)

17. Rosin, N. L. et al. SARS-CoV-2 infection of circulating immune cells is not responsible for virus dissemination in severe COVID-19 patients. Preprint at https://www.biorxiv.org/content/10.1101/2021.01.19.427282v1.full (2021).

18. World Health Organization. Clinical management of severe acute respiratory infection (SARI) when COVID-19 disease is suspected. https://www.who.int/ docs/default-source/coronaviruse/clinical-management-of-novel-cov.pdf (2020).

19. Alberta Health Services. Influenza Immunization: Data \& Statistics. https:// www.albertahealthservices.ca/influenza/influenza.aspx\#data

20. Ranieri, V. M. et al. Acute respiratory distress syndrome: the Berlin Definition. JAMA 307, 2526-2533 (2012).

21. Mehta, P. et al. COVID-19: consider cytokine storm syndromes and immunosuppression. Lancet 395, 1033-1034 (2020).

22. Feng, Z. et al. Early prediction of disease progression in COVID-19 pneumonia patients with chest CT and clinical characteristics. Nat. Commun 11, 4968 (2020).

23. Wu, C. et al. Risk factors associated with acute respiratory distress syndrome and death in patients with coronavirus disease 2019 pneumonia in Wuhan, China. JAMA Intern. Med. 180, 934-943 (2020).

24. Barnes, B. J. et al. Targeting potential drivers of COVID-19: neutrophil extracellular traps. J. Exp. Med. 217, e20200652 (2020).

25. Busse, D. et al. Interferon-induced protein 44 and interferon-induced protein 44-like restrict replication of respiratory syncytial virus. J. Virol. 94, e00297-00220 (2020).

26. Bergen, V., Lange, M., Peidli, S., Wolf, F. A. \& Theis, F. J. Generalizing RNA velocity to transient cell states through dynamical modeling. Nat. Biotechnol. 38, 1408-1414 (2020)

27. La Manno, G. et al. RNA velocity of single cells. Nature 560, 494-498 (2018).

28. Qiu, X. et al. Mapping transcriptomic vector fields of single cells. Preprint at https://www.biorxiv.org/content/10.1101/696724v2 (2021).

29. Chen, J. S. et al. Non-steroidal anti-inflammatory drugs dampen the cytokine and antibody response to SARS-CoV-2 infection. J. Virol. 95, e00014-21 (2021).

30. Takashima, A. \& Yao, Y. Neutrophil plasticity: acquisition of phenotype and functionality of antigen-presenting cell. J. Leukoc. Biol. 98, 489-496 (2015).

31. Ledford, J. G., Kovarova, M. \& Koller, B. H. Impaired host defense in mice lacking ONZIN. J. Immunol. 178, 5132 (2007).

32. Rørvig, S. et al. Ficolin-1 is present in a highly mobilizable subset of human neutrophil granules and associates with the cell surface after stimulation with fMLP. J. Leukoc. Biol. 86, 1439-1449 (2009). 
33. Zhang, Q. et al. Life-threatening COVID-19: defective interferons unleash excessive inflammation. Med. (N Y) 1, 14-20 (Elsevier, 2020).

34. Zhang, Q. et al. Inborn errors of type I IFN immunity in patients with life-threatening COVID-19. Science 370, eabd4570 (2020).

35. Aibar, S. et al. SCENIC: single-cell regulatory network inference and clustering. Nat. Methods 14, 1083-1086 (2017).

36. Hsu, J. \& Sage, J. Novel functions for the transcription factor E2F4 in development and disease. Cell Cycle 15, 3183-3190 (2016).

37. Shu, T. et al. Plasma proteomics identify biomarkers and pathogenesis of COVID-19. Immunity 53, 1108-1122 (2020).

38. Park, J. et al. In-depth blood proteome profiling analysis revealed distinct functional characteristics of plasma proteins between severe and non-severe COVID-19 patients. Sci. Rep. 10, 22418 (2020).

39. Demichev, V. et al. A time-resolved proteomic and prognostic map of COVID-19. Cell Syst. 12, 780-794 (2021).

40. Mahler, M., Meroni, P. L., Infantino, M., Buhler, K. A. \& Fritzler, M. J. Circulating calprotectin as a biomarker of COVID-19 severity. Expert Rev. Clin. Immunol. 17, 431-443 (2021).

41. Zhao, Y. et al. Clonal expansion and activation of tissue-resident memory-like $\mathrm{T}_{\mathrm{H}} 17$ cells expressing GM-CSF in the lungs of patients with severe COVID-19. Sci. Immunol. 6, eabf6692 (2021).

42. Wilk, A. J. et al. Multi-omic profiling reveals widespread dysregulation of innate immunity and hematopoiesis in COVID-19. J. Exp. Med. 218, e20210582 (2021).

43. Overmyer, K. A. et al. Large-scale multi-omic analysis of COVID-19 severity. Cell Syst. 12, 23-40 (2021).

44. Nestorowa, S. et al. A single-cell resolution map of mouse hematopoietic stem and progenitor cell differentiation. Blood 128, e20-e31 (2016).

45. Vago, J. P. et al. Annexin A1 modulates natural and glucocorticoid-induced resolution of inflammation by enhancing neutrophil apoptosis. J. Leukoc. Biol. 92, 249-258 (2012).

46. Oliveira, L. G. et al. Annexin A1 is involved in the resolution of inflammatory responses during Leishmania braziliensis infection. J. Immunol. 198, 3227 (2017)

47. Uhel, F. et al. Early expansion of circulating granulocytic myeloid-derived suppressor cells predicts development of nosocomial infections in patients with sepsis. Am. J. Respir. Crit. Care Med. 196, 315-327 (2017).

48. Arlauckas, S. P. et al. Arg1 expression defines immunosuppressive subsets of tumor-associated macrophages. Theranostics 8, 5842-5854 (2018).

49. Derakhshani, A. et al. Arginase 1 ( $\operatorname{Arg} 1)$ as an up-regulated gene in COVID-19 patients: a promising marker in COVID-19 immunopathy. J. Clin. Med. 10, 1051 (2021).
50. Kelly-Scumpia, K. M. et al. ER stress regulates immunosuppressive function of myeloid derived suppressor cells in leprosy that can be overcome in the presence of IFN- $\gamma$. iScience 23, 101050 (2020).

51. Okun, J. G. et al. Molecular regulation of urea cycle function by the liver glucocorticoid receptor. Mol. Metab. 4, 732-740 (2015).

52. Perretti, M. \& D'Acquisto, F. Annexin A1 and glucocorticoids as effectors of the resolution of inflammation. Nat. Rev. Immunol. 9, 62-70 (2009).

53. Peckham, H. et al. Male sex identified by global COVID-19 metaanalysis as a risk factor for death and ITU admission. Nat. Commun. 11, 6317 (2020).

54. Park, A. \& Iwasaki, A. Type I and type III interferons-induction, signaling, evasion, and application to combat COVID-19. Cell Host Microbe 27, 870-878 (2020)

55. Gupta, S. et al. Sex differences in neutrophil biology modulate response to type I interferons and immunometabolism. Proc. Natl Acad. Sci. USA 117, 16481 (2020).

56. Monk, P. D. et al. Safety and efficacy of inhaled nebulised interferon beta-1a (SNG001) for treatment of SARS-CoV-2 infection: a randomised, double-blind, placebo-controlled, phase 2 trial. Lancet Respir. Med. 9, 196-206 (2021).

57. Wang, N. et al. Retrospective multicenter cohort study shows early interferon therapy is associated with favorable clinical responses in COVID-19 patients. Cell Host Microbe 28, 455-464 (2020).

58. Schulte-Schrepping, J. et al. Severe COVID-19 is marked by a dysregulated myeloid cell compartment. Cell 182, 1419-1440 (2020).

Publisher's note Springer Nature remains neutral with regard to jurisdictional claims in published maps and institutional affiliations.

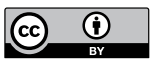

Open Access This article is licensed under a Creative Commons Attribution 4.0 International License, which permits use, sharing, adaptation, distribution and reproduction in any medium or format, as long as you give appropriate credit to the original author(s) and the source, provide a link to the Creative Commons license, and indicate if changes were made. The images or other third party material in this article are included in the article's Creative Commons license, unless indicated otherwise in a credit line to the material. If material is not included in the article's Creative Commons license and your intended use is not permitted by statutory regulation or exceeds the permitted use, you will need to obtain permission directly from the copyright holder. To view a copy of this license, visit http://creativecommons. org/licenses/by/4.0/.

(c) The Author(s) 2021 


\section{Methods}

Patient enrollment. We recruited six bacterial ARDS ( $n=5$ at $\mathrm{t} 1$ and $n=4$ at $\mathrm{t} 2$ ), eight non-dexamethasone COVID-19 ARDS ( $n=8$ at $\mathrm{t} 1$ and $n=4$ at $\mathrm{t} 2$ ) and six dexamethasone-treated COVID-19 ARDS ( $n=6$ at $\mathrm{t} 1$ and $n=3$ at t2) patients who were admitted to the ICU (Supplementary Table 2). All patients were enrolled after admission to any of the four adult ICUs at South Health Campus, Rockyview General Hospital, Foothills Medical Center or Peter Lougheed Center in Calgary, Alberta, Canada (Extended Data Fig. 1). Patient admission to the ICU was determined by the attending ICU physician based on the need for life-sustaining interventions, monitoring and life support. The research teams did not participate in clinical decisions. Study inclusion required a minimum age of 18 years of age, the ability to provide consent or, for most participants, the ability of a surrogate decision-maker to provide regained capacity consent. All participants required an arterial catheter for blood draws, but the insertion of this catheter was at the discretion of the attending medical team. Patients with COVID-19 ARDS required a positive clinical RNA COVID-19 test before enrollment and evidence of bilateral lung infiltrates and hypoxemia consistent with ARDS. All patients with COVID-19 ARDS were treated with empiric antibiotics. At the time of sample collections, all enrolled patients who were positive for COVID-19 were culture negative for concurrent bacterial infections in the blood, urine and sputum. The bacterial ARDS cohort required a negative COVID-19 test and a definitive microbiological diagnosis of bacterial pneumonia with chest imaging consistent with a diagnosis of ARDS. Patients were excluded from our study if they (1) were on immunosuppressive therapies; (2) had established autoimmune disease; or (3) had active malignancy. Because tocilizumab, remdesivir or any other immunomodulatory agents were not approved for use in patients with severe COVID-19 in Alberta over the time span of this study, participants did not receive these medications. Starting on 1 June 2020, all patients with COVID-19 received dexamethasone ( $6 \mathrm{mg}$ per day) upon hospital admission, as dexamethasone became the standard of care at that time. Although patients with bacterial ARDS received appropriate antibiotic treatments, none was prescribed immunosuppressive or steroid therapy. All patients with bacterial ARDS had lung infections caused by Gram-positive cocci (four S. aureus and two S. pneumoniae). To be included, participants were required to have a definitive diagnosis and appropriate consent and samples collected within $72 \mathrm{~h}$ of admission to the ICU. t1 refers to the first blood draw, whereas $\mathrm{t} 2$ was a repeat blood draw taken $7 \mathrm{~d}$ after $\mathrm{t} 1$, if the participant remained in the ICU and had an arterial catheter. For each participant, whole blood was collected via the arterial catheter and immediately processed for analysis. Healthy blood donors were recruited by university-wide advertisement and required that participants were (1) not on immunomodulatory medications; (2) were asymptomatic for COVID-19; (3) did not receive vaccination against SARS-CoV-2; and (4) did not have underlying immune disorders.

Epidemiological analysis. For this study, we used the Alberta provincial eCritical Oracle-based analytics database (Tracer) to query and extract Alberta COVID$19 \mathrm{ICU}$ cases and volumes ${ }^{59}$. Aggregate data from 16 individual adult ICUs were obtained over the study periods. Data for dexamethasone administration could not be captured at an individual level; therefore, we queried the database for patients admitted to the ICU before dexamethasone became standard of care in our province (pre-dexamethasone era: 1 January 2020 to 31 May 2020) versus dexamethasone as standard of care for severe COVID-19 (1 June 2020 to 31 May 2021). Tocilizumab was approved for use in Alberta on 11 March 2021, and a small supply (150 doses) was obtained for patients with severe COVID-19 after this date.

Human study ethics. All work with humans was approved by the Conjoint Health Research Ethics Board at the University of Calgary (Ethics ID: REB20-0481) and is consistent with the Declaration of Helsinki.

\section{Serum cytokine assessment. Cytokines, chemokines and soluble cytokine} receptors were quantitated on multiplex arrays that included a 65 MILLIPLEX cytokine/chemokine (6Ckine, BCA-1, CTACK, EGF, ENA-78, Eotaxin, Eotaxin-2, Eotaxin-3, FGF-2, Flt-3L, Fractalkine, G-CSF, GM-CSF, GRO, I-309, IFN- $\alpha 2$, IFN- $\gamma$, IL-1 $\alpha$, IL-1 $\beta$, IL-1ra, IL-2, IL-3, IL-4, IL-5, IL-6, IL-7, IL-8, IL-9, IL-10, IL-12 (p40), IL-12 (p70), IL-13, IL-15, IL-16, IL-17A, IL-18, IL-20, IL-21, IL-23, IL-28a, IL-33, IP-10, LIF, MCP-1, MCP-2, MCP-3, MCP-4, MDC, MIP-1 $\alpha$, MIP$1 \beta$, MIP-1d, PDGF-AA, PDGF-AB/BB, RANTES, SDF- $1 \alpha$, SDF-1 $\beta$, sCD40L, SCF, TARC, TGFa, TNFa, TNFb, TPO, TRAIL, TSLP, VEGF) and a 14 MILLIPLEX soluble cytokine (sCD30, sEGFR, sgp130, sIL-1RI, sIL-1RII, sIL-2Ra, sIL-4R, sIL6R, sRAGE, sTNF RI, sTNF RII, sVEGF R1, sVEGF R2 and sVEGF R3) (Millipore Sigma) on a Luminex 200 luminometer. EDTA plasma samples were collected from each patient by venipuncture after a standard operating protocol and stored at -80 ${ }^{\circ} \mathrm{C}$ until tested. Each run included a full range of calibrators. The Mann-Whitney $U$-test was used to compare groups, and $P$ values were adjusted for multiple comparisons using the Holm-Sídak stepdown method with $\alpha$ set to 0.05 .

\section{Shotgun proteomics using liquid chromatography with tandem mass} spectrometry. The serum of patients with COVID-19 (COVID-19 non-dexamethasone $=9$ and COVID-19 dexamethasone $=4$ ) and bacterial ARDS controls $(n=6)$ were collected. The total protein concentrations were determined by Pierce BCA Protein Assay Kit (23225, Thermo Fisher Scientific). A trichloroacetic acid/acetone protocol was used to pellet $100 \mu \mathrm{g}$ of proteins per sample $\left(14,000 \mathrm{~g}, 15 \mathrm{~min}, 4^{\circ} \mathrm{C}\right)$, followed by air drying for $2 \mathrm{~min}$. Samples were subjected to a quantitative proteomics workflow as per supplier (Thermo Fisher Scientific) recommendations. Samples were reduced in $200 \mathrm{mM}$ tris(2-carboxyethyl) phosphine for $1 \mathrm{~h}$ at $55^{\circ} \mathrm{C}$, and reduced cysteines were alkylated by incubation with iodoacetamide solution $(50 \mathrm{mM})$ for $20 \mathrm{~min}$ at room temperature. Samples were precipitated by acetone/methanol, and $600 \mu \mathrm{l}$ of ice-cold acetone was added, followed by incubation at $-20^{\circ} \mathrm{C}$ overnight. A protein pellet was obtained by centrifugation $\left(8,000 \mathrm{~g}, 10 \mathrm{~min}, 4^{\circ} \mathrm{C}\right)$, followed by acetone drying $(2 \mathrm{~min})$. The precipitated pellet was resuspended in $100 \mu \mathrm{l}$ of $50 \mathrm{mM}$ triethylammonium bicarbonate buffer, followed by tryptase digestion ( $5 \mu \mathrm{g}$ of trypsin per $100 \mu \mathrm{g}$ of protein) overnight at $37^{\circ} \mathrm{C}$. TMT-6plex Isobaric Labeling Reagents (90061, Thermo Fisher Scientific) were resuspended in anhydrous acetonitrile and added to each sample (41 $\mu$ l of TMT-6plex per 100- $\mu$ l sample) and incubated at room temperature for $1 \mathrm{~h}$. The TMT labeling reaction was quenched by $2.5 \%$ hydroxylamine for $15 \mathrm{~min}$ at room temperature. TMT-labeled samples were combined and acidified in $100 \%$ trifluoroacetic acid to $\mathrm{pH}<3.0$ and subjected to $\mathrm{C} 18$ chromatography (Sep-Pak) according to manufacturer recommendations. Samples were stored at $-80^{\circ} \mathrm{C}$ before lyophilization, followed by resuspension in $1 \%$ formic acid before liquid chromatography with tandem mass spectrometry (LC-MS/MS) analysis.

Tryptic peptides were analyzed on an Orbitrap Fusion Lumos Tribrid mass spectrometer (Thermo Fisher Scientific) operated with Xcalibur (version 4.0.21.10) and coupled to a Thermo Fisher Scientific Easy-nLC (nanoflow liquid chromatography) 1200 system. Tryptic peptides $(2 \mu \mathrm{g})$ were loaded onto a C18 trap $(75 \mu \mathrm{m} \times 2 \mathrm{~cm}$; Acclaim PepMap 100, P/N 164946, Thermo Fisher Scientific) at a flow rate of $2 \mu \mathrm{min}^{-1}$ of solvent A ( $0.1 \%$ formic acid in LC-MS-grade water). Peptides were eluted using a 120 -min gradient from $5 \%$ to $40 \%$ (5\% to $28 \%$ in $105 \mathrm{~min}$, followed by an increase to $40 \%$ B in $15 \mathrm{~min}$ ) of solvent B ( $0.1 \%$ formic

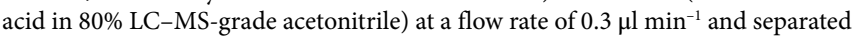
on a C18 analytical column $(75 \mu \mathrm{m} \times 50 \mathrm{~cm}$; PepMap RSLC C18, P/N ES803A, Thermo Fisher Scientific). Peptides were then electrosprayed using $2.1 \mathrm{kV}$ voltage into the ion transfer tube $\left(300^{\circ} \mathrm{C}\right)$ of the Orbitrap Lumos operating in positive mode. The Orbitrap first performed a full MS scan at a resolution of 120,000 full width at half maximum to detect the precursor ion having a $\mathrm{m} / \mathrm{z}$ between 375 and 1,575 and $\mathrm{a}+2$ to +4 charge. The Orbitrap AGC (automatic gain control) and the maximum injection time were set at $4 \times 10^{5}$ and $50 \mathrm{~ms}$, respectively. The Orbitrap was operated using the top speed mode with a 3-s cycle time for precursor selection. The most intense precursor ions presenting a peptidic isotopic profile and having an intensity threshold of at least $2 \times 10^{4}$ were isolated using the quadrupole (isolation window $(\mathrm{m} / \mathrm{z}$ ) of 0.7 ) and fragmented using higher-energy C-trap dissociation ( $38 \%$ collision energy) in the ion routing multipole. The fragment ions (MS2) were analyzed in the Orbitrap at a resolution of 15,000. The AGC and the maximum injection time were set at $1 \times 10^{5}$ and $105 \mathrm{~ms}$, respectively. The first mass for the MS2 was set at 100 to acquire the TMT reporter ions. Dynamic exclusion was enabled for $45 \mathrm{~s}$ to avoid acquisition of the same precursor ion having a similar $m / z( \pm 10$ p.p.m.).

Leukocyte and lymphocyte isolation. For lymphocyte isolation, whole blood heparinized vacutubes were used. To isolate lymphocytes by immunomagnetic negative selection, $100 \mu \mathrm{l}$ of Isolation Cocktail and $100 \mu \mathrm{l}$ of Rapid Spheres (EasySep Direct Human Total Lymphocytes Isolation Kit, 19655, STEMCELL Technologies) were added to $2 \mathrm{ml}$ of whole blood. After mixing and 5-min incubation at room temperature, the sample volumes were topped up to $2.5 \mathrm{ml}$ with $0.04 \%$ BSA in PBS. The diluted sample was incubated in the magnet without a lid for $5 \mathrm{~min}$ at room temperature, and negatively selected lymphocytes were decanted into a new 5-ml polystyrene tube. Except for the addition of Isolation Cocktail, all steps were repeated once. The final lymphocyte cell suspension was transferred to a $15-\mathrm{ml}$ polypropylene tube, and a volume of $5 \mathrm{ml}$ of $0.04 \%$ BSA in PBS was added to the sample. Lymphocytes were precipitated by centrifugation for $5 \mathrm{~min}$ at $300 \mathrm{~g}$; the supernatant was discarded; and cells were resuspended in $5 \mathrm{ml}$ of $0.04 \%$ BSA in PBS. This step was repeated, and cells were resuspended in $100 \mu$ of PBS $+0.04 \%$ BSA. Cell density was quantified with a hemacytometer; cell viability was assessed with trypan blue staining (T8154, Sigma-Aldrich); and 7,500 live lymphocytes were transferred to a sterile $1.5-\mathrm{ml}$ microcentrifuge tube.

For leukocyte isolation, $1 \mathrm{ml}$ of whole blood from heparin-containing vacutubes was transferred to $5-\mathrm{ml}$ polystyrene round-bottom tubes, and $12 \mu \mathrm{l}$ of $0.5 \mathrm{M}$ EDTA was added. Next, $2 \%$ FBS in PBS ( $1 \mathrm{ml})$ and $50 \mu \mathrm{l}$ of EasySep RBC Depletion spheres (EasySep RBC Depletion Reagent, 18170, STEMCELL Technologies) were added to immunomagnetically deplete red blood cells. After $5 \mathrm{~min}$ of magnet incubation at room temperature, cell suspension containing leukocytes was decanted into a new 5-ml polystyrene tube. To ensure complete removal of red blood cells, red blood cell depletion was repeated, and cell suspension containing leukocytes was decanted into a new $15-\mathrm{ml}$ polypropylene tube. Leukocytes were precipitated by centrifugation at $300 \mathrm{~g}$ for $5 \mathrm{~min}$ at $20^{\circ} \mathrm{C}$ and resuspended in $5 \mathrm{ml}$ of $0.04 \%$ BSA in PBS. This step was repeated, and leukocytes were resuspended in $2 \mathrm{ml}$ of $0.04 \%$ BSA in PBS. Cell viability and cell density were assessed, and 7,500 live leukocytes were transferred to the microcentrifuge tube containing the lymphocyte cell suspension in a total volume of $50 \mu \mathrm{l}$ of $0.04 \%$ BSA in PBS. 
Immunocytochemistry and immunohistochemistry. Isolated leukocyte and lymphocyte samples were fixed in $4 \%$ paraformaldahyde in PBS $(0.2 \mathrm{mM}$ and $\mathrm{pH} 7.4$ ) and spun in a cytocentrifuge $(8 \mathrm{~min}$ at $300 \mathrm{~g}$ ) onto coated slides. Slides were permeabilized and blocked with $10 \%$ normal donkey serum in PBS (with $0.5 \%$ Triton X-100), and primary antibodies (S100A8/9, Abcam, ab22506; IFITM1, Abcam, ab233545) were incubated at $4{ }^{\circ} \mathrm{C}$ overnight, followed by incubation with donkey anti-rabbit Alexa Fluor 488 (Invitrogen, A32790) or anti-mouse Alexa Fluor 555 (Invitrogen, A31570) for $1 \mathrm{~h}$ at room temperature. Slides were sequentially stained with CD24 (Abcam, ab202073) on the same slides for $1 \mathrm{~h}$ at room temperature, followed by donkey anti-rabbit Alexa Fluor 647 (Invitrogen, A31573). Imaging was done using a VS-120 slide scanner (Olympus), and high-resolution imaging was done using an SP8 spectral confocal microscope (Leica). Image processing was completed in Fiji (version 2.1.0 ${ }^{60}$.

scRNA-seq library construction, alignment and quality control. A total of 15,000 single cells (containing an equal proportion of leukocytes and lymphocytes) were loaded for partitioning using 10x Genomics NextGEM Gel Bead emulsions ( 3 ' gene expression kit, version 3.1). All samples were processed as per the manufacturer's protocol (both PCR amplification steps were run $12 \times$ ). Quality control (QC) of resulting libraries and quantification was performed using TapeStation D1000 ScreenTape assay (Agilent). Sequencing was performed using Illumina NovaSeq S2 and SP 100 cycle dual lane flow cells over multiple rounds to ensure that each sample received at least 32,000 reads per cell. Sequencing reads were aligned using the CellRanger 3.1.0 pipeline ${ }^{61}$ to the standard pre-built GRCh38 reference genome. Samples that passed alignment QC were aggregated into single datasets using CellRanger aggr with between-sample normalization to ensure that each sample received an equal number of mapped reads per cell. Aggregated non-dexamethasone-treated COVID-19 $(n=12)$ and bacterial ARDS $(n=9)$ samples recovered $1,872,659$ cells that were sequenced to 38,410 post-normalization reads per cell. Likewise, aggregated COVID-19 samples with $(n=9)$ or without $(n=12)$ dexamethasone recovered $1,748,551$ single cells sequenced to 51,415 post-normalization reads per cell. Aggregated healthy samples recovered 19,816 cells, including 1,912 post-QC neutrophils $(n=5)$.

scRNA-seq computational analyses and workflows. Filtered feature barcode HDF5 matrices from aggregated datasets were imported into the R package Seurat (version 3.9 and version 4) for normalization, scaling, integration, multi-modal reference mapping, Louvain clustering, dimensionality reduction, differential expression analysis and visualization ${ }^{62}$. In brief, cells with abnormal transcriptional complexity (fewer than 500 UMIs, more than 25,000 UMIs or greater than $25 \%$ of mitochondrial reads) were considered artifacts and were removed from subsequent analysis. Because granulocytes have relatively low RNA content (due to high levels of RNases), QC thresholds were informed by Xie et. al. ${ }^{8}$. Cell identity was classified by mapping single-cell profiles to the recently published peripheral blood mononuclear cell single-cell joint RNA/CITE-seq multi-omic reference (Azimuth) ${ }^{63}$

Annotation of neutrophil states. Because the Azimuth reference does not contain granulocytes that would automate neutrophil annotations within queried datasets, neutrophil clusters were manually annotated by querying known markers (that is, CSF3R, S100A8, S100A9, MMP8, MMP9, ELANE and MPO) ${ }^{64}$ and were corroborated using the R package SingleR ${ }^{65}$. Neutrophil states were defined by grouping unsupervised (Louvain at default resolution) subclusters based on two overlapping criteria: (1) scVelo-inferred neutrophil maturity and (2) by corroborating gene expression and SCENIC-inferred GRN signatures with previous human and rodent neutrophil scRNA-seq studies. Immature neutrophils were defined as $\mathrm{CD} 24^{+} \mathrm{ARG}^{+} \mathrm{ELANE}^{+} \mathrm{MPO}^{+} \mathrm{ATF} 4^{\mathrm{GRN}-a c t i v e} J \mathrm{JP}$ $2^{\mathrm{GRN}-a c t i v e}$ neutrophils ${ }^{7,8,58,66}$ that were reproducibly assigned as 'root cells' in scVelo-based latent time pseudo-ordering. IFN ${ }^{\text {active }}$ neutrophils were defined by preferential mRNA splicing (positive velocity) and expression of ISGs, such as IFITM1/2, IFIT1/2/3, ISG15/20 and IFI6/27/44/44L $\mathrm{L}^{6,55,67}$. PG active neutrophils were distinguished by preferential splicing of PTGS2/COX2 (as well as expression for prostaglandin transport LST1 $)^{55}$ and included a subset that expressed high levels of IL-1 $\beta$ decoy receptor IL-1R2 (ref. ${ }^{42}$ ). Lastly, IL-7R ${ }^{+}$neutrophils (a small but distinct subset that might be of thymic origin ${ }^{68}$ ) expressed high levels of ribosomal subunit genes (for example, RPL5/7A/8/13/18/19/23/24/27/P0) that are highly reminiscent of 'ribosomal ${ }^{\text {hi }}$-specific cluster 7 ' identified previously ${ }^{58}$.

Statistical approach for comparing cell proportions. To test whether cell composition was changed due to infection type (COVID-19 versus bacterial ARDS) or treatment group (dexamethasone versus non-dexamethasone), a generalized linear mixed-effects model was employed where infection type and treatment group were considered fixed, and individual patients were considered random effect. Fitting was done with Laplace approximation using the 'glmer' function in the 'lme4' R package (version 1.1-27.1) ${ }^{69}$, and $P$ values were calculated using the R package 'car' (version 3.0-11). Box plots comparing cell type composition were generated using the ggplot 2 package. Because a subset of patients sampled at $\mathrm{t} 1$ was discharged from ICU before $\mathrm{t} 2$ collection (non-random or non-ignorable missing data), we limited statistical comparisons to between-group comparisons within one time point (for example, COVID-19 tl versus bacterial
ARDS $t 1$ or dexamethasone-treated $t 1$ versus non-dexamethasone-treated $t 1)$ and did not estimate temporal differences across $\mathrm{t} 1$ and $\mathrm{t} 2$.

Inferring cell communication networks. Differential cell-cell interaction networks were reconstructed using the Connectome R toolkit version 0.2 .2 (ref. ${ }^{70}$ ) and CellChat version 1.0.0 (ref. ${ }^{71}$ ). In brief, DifferentialConnectome queried Seurat (version 3.9 and version 4) R objects housing datasets integrated by infection type and dexamethasone status to define nodes and edges for downstream network analysis. The total numbers of interactions and interaction strengths were calculated using the compareInteractions function in CellChat. The differential edge list was passed through CircosDiff (a wrapper around the R package 'circlize') and netVisual_chord_gene in CellChat to filter receptor ligand edges and generate Circos plots.

Consensus DEGs and perturbation scores. DEGs were those with an average $\operatorname{logFC}$ greater than 0.25 (adjusted $P<0.05$ ) as determined by Seurat (version 3.9 and version 4) Wilcoxon rank-sum test. Consensus stacked bars showing cumulative logFCs (colored by individual sample contributions) were generated using the constructConsensus function ${ }^{7}$ for genes exhibiting reproducible changes across patients ( $>3$ for 72 -h comparisons and $>2$ for 7 - $d$ comparisons). Gene set enrichment analyses of consensus DEGs were performed using gProfiler's g:GOSt ( $P$ value cutoff $<0.05)$. A cell-state-specific 'perturbation score' was calculated to reflect the magnitude of response elicited by factoring in number and cumulative FC of consensus DEGs. Perturbation scores were visualized using Nebulosa (version 1.0.2)-generated density plots ${ }^{72}$

Constructing cellular trajectories using RNA velocity. Analysis of neutrophil trajectories was performed by realigning CellRanger count-generated BAMs with the RNA velocity command line tool ${ }^{27}$ using the run $10 \mathrm{x}$ command and human (GRCh38) annotations. The output loom files containing spliced and unspliced counts were combined to compare neutrophils in COVID-19 with bacterial ARDS controls and dexamethasone-treated with non-treated patients with COVID-19. For both analyses, combined looms were imported into Seurat (version 3.9 and version 4) using the ReadVelocity function in SeuratWrappers version 0.2.0, normalized using SCTransform (version 0.3 .2$)^{73}$, reduced and projected onto a UMAP and exported as an $\mathrm{H} 5$ file using the SaveH5Seurat function. Counts stored in $\mathrm{H} 5$ files were imported, filtered and normalized as recommended in the scVelo (version 0.2 .1 ) workflow ${ }^{26}$. RNA velocities were estimated using stochastic and dynamical models. Because both models yielded similar results, a stochastic model was used as default for all subsequent analyses. Calculations stored in AnnData's metadata were exported as CSV files, and kernel density lines depicting Velocity-inferred latent time distribution were plotted with ggplot2 (version 3.1.1)

Gene regulatory network and Gene Ontology enrichment. SCENIC ${ }^{35}$ was employed to infer regulatory interactions between TFs and their targetome by calculating and pruning co-expression modules. In brief, neutrophils were subsetted from scVelo-realigned Seurat (version 3.9 and version 4) object and processed using default and recommended parameters specified in SCENIC's vignette (https://github.com/aertslab/SCENIC) using the hg19 RcisTarget reference. Regulon activity scores (in '3.4_regulonAUC.Rds', an output of the SCENIC workflow) were added to scVelo object (using the CreateAssayObject function) to jointly project trajectory and TF activity onto the same UMAP embeddings. Consensus stacked bars showing cumulative logFC of AUCell scores for each TF (colored by individual sample contributions) were generated by modifying the constructConsensus function ${ }^{7}$ for the SCENIC assay. The targetome of TFs predicted as drivers of neutrophil states (stored in '2.6_regulons_asGeneSet. Rds') was profiled using g:Profiler's functional enrichment analysis, and genes intersecting with the INF pathway were plotted using iRegulon (Cytoscape plugin $)^{74}$. Gene Ontology term enrichment analysis was performed using the Seurat (version 3.9 and version 4) DEenrichRPlot function, a wrapper around the Ma'ayan lab's Enrichr ${ }^{75}$, where DEGs were calculated using the Wilcoxon rank-sum test, and a maximum of 300 genes were provided as input to Enrichr.

\section{Comparing scRNA-seq findings with published datasets. To test whether} dexamethasone-suppressed neutrophil genes at $\mathrm{t} 1$ and $\mathrm{t} 2$ (Supplementary Table 5) predicted COVID-19 mortality, we repurposed methods described in ref. ${ }^{42}$ and employed whole blood bulk RNA-seq datasets generated in ref. ${ }^{43}$ as a validation cohort of 103 samples (where 17 were fatal). In brief, each of the 103 samples was scored by the aggregated expression of dexamethasone-suppressed neutrophil consensus genes at $\mathrm{t} 1$ and $\mathrm{t} 2$ using Seurat (version 3.9 and version 4) AddModuleScore(). Dexamethasone-suppressed module scores were used as the predictor variable, and 28-d mortality was used as the response variable to construct a receiver operating characteristic (ROC) curve using pROC's roc() function. To infer bronchoalveolar neutrophil composition in severe and moderate COVID-19 (ref. ${ }^{11}$ ) and across bacterial pneumonia and COVID-19 (ref. ${ }^{41}$ ), neutrophils $\left(\mathrm{CSF} 3 \mathrm{R}^{+}, \mathrm{S} 100 \mathrm{~A}^{+}\right.$and $\mathrm{S} 100 \mathrm{~A} 9^{+}$) captured in BALF scRNA-seq datasets were projected onto our peripheral blood reference using mutual nearest neighbor anchoring (FindTransferAnchors) and the identity transferring (TransferData and AddMetaData) strategy implemented in Seurat version 4 (ref. ${ }^{62}$ ). 
COVID neutrophil atlas. To enable intuitive exploration of single-cell datasets, a web portal (http://biernaskielab.ca/COVID_neutrophil or http://biernaskielab. com/COVID_neutrophil) was built using RShiny (version 1.1.0), shinyLP (version 1.1.2) and shinythemes (version 1.1.2) packages.

Reporting Summary. Further information on research design is available in the Nature Research Reporting Summary linked to this article.

\section{Data availability}

eCritical is a secure patient database that is not publicly accessible. Requests for access to patient-related data-de-identified, summary or patient-level datamust be approved by eCritical with an appropriate ethics protocol and might require approval by Alberta Health Services. scRNA-seq datasets are available at the National Center of Biotechnology Information's Gene Expression Omniobus (which automatically makes Sequence Read Archive deposit) under the following accession number: GSE157789. Single-cell datasets can be further explored on our companion portal at http://biernaskielab.ca/COVID_neutrophil or http:// biernaskielab.com/COVID neutrophil. Velocyto-generated loom files and processed $\mathrm{R}$ objects are available for reanalysis from https://doi.org/10.6084/ m9.figshare.14330795. Whole blood bulk RNA-seq datasets employed as an independent validation cohort were downloaded from GSE157103. BALF scRNA-seq datasets from severe and moderate COVID-19 were downloaded from GSE145926. Processed BALF scRNA-seq objects from patients with bacterial pneumonia and COVID-19 (archived at GSE167118) were downloaded from the authors' archive: https://figshare.com/articles/dataset/_/13608734. Mass spectrometry datasets are available at the ProteomeXchange Consortium in the PRIDE partner repository with identifier PXD028429.

\section{Code availability}

All analyses were performed using publicly available software as described in the Methods section. Raw scripts are available upon reasonable request.

\section{References}

59. Brundin-Mather, R. et al. Secondary EMR data for quality improvement and research: a comparison of manual and electronic data collection from an integrated critical care electronic medical record system. J. Crit. Care 47, 295-301 (2018).

60. Schindelin, J. et al. Fiji: an open-source platform for biological-image analysis. Nat. Methods 9, 676-682 (2012).

61. Zheng, G. X. Y. et al. Massively parallel digital transcriptional profiling of single cells. Nat. Commun. 8, 14049 (2017)

62. Stuart, T. et al. Comprehensive integration of single-cell data. Cell 177, 1888-1902 (2019).

63. Hao, Y. et al. Integrated analysis of multimodal single-cell data. Cell 184, 3573-3587.e29 (2020).

64. Zilionis, R. et al. Single-cell transcriptomics of human and mouse lung cancers reveals conserved myeloid populations across individuals and species. Immunity 50, 1317-1334 (2019).

65. Aran, D. et al. Reference-based analysis of lung single-cell sequencing reveals a transitional profibrotic macrophage. Nat. Immunol. 20, 163-172 (2019).

66. Reusch, N. et al. Neutrophils in COVID-19. Front. Immunol. 12, 652470-652470 (2021)

67. Shaath, H., Vishnubalaji, R., Elkord, E. \& Alajez, N. M. Single-cell transcriptome analysis highlights a role for neutrophils and inflammatory macrophages in the pathogenesis of severe COVID-19. Cells 9, 2374 (2020).

68. Schlenner, S. M. et al. Fate mapping reveals separate origins of T cells and myeloid lineages in the thymus. Immunity 32, 426-436 (2010).

69. Bates, D., Mächler, M., Bolker, B. \& Walker, S. Fitting linear mixed-effects models using lme4. J. Stat. Soft. 67, 1-48 (2015).
70. Raredon, M. S. B. et al. Connectome: computation and visualization of cell-cell signaling topologies in single-cell systems data. Preprint at https:// www.biorxiv.org/content/10.1101/2021.01.21.427529v1 (2021).

71. Jin, S. et al. Inference and analysis of cell-cell communication using CellChat. Nat. Commun. 12, 1088 (2021).

72. Alquicira-Hernandez, J. \& Powell, J. E. Nebulosa recovers single-cell gene expression signals by kernel density estimation. Bioinformatics https://doi. org/10.1093/bioinformatics/btab003 (2021).

73. Hafemeister, C. \& Satija, R. Normalization and variance stabilization of single-cell RNA-seq data using regularized negative binomial regression. Genome Biol. 20, 296 (2019).

74. Janky, R. S. et al. iRegulon: from a gene list to a gene regulatory network using large motif and track collections. PLoS Comput. Biol. 10, e1003731 (2014).

75. Kuleshov, M. V. et al. Enrichr: a comprehensive gene set enrichment analysis web server 2016 update. Nucleic Acids Res. 44, W90-W97 (2016).

\section{Acknowledgements}

This work was funded by a FastGrant from the Thistledown Foundation (J.B. and B.Y.) and the Calgary Firefighters Burn Treatment Society (J.B.). S.S. received CIHR Vanier, Alberta Innovates and Killam doctoral scholarships. E.L received an Alberta Children's Hospital Research Institute postdoctoral fellowship. B.G.Y is a Tier II Canada Research Chair in Pulmonary, Immunology, Inflammation and Host Defence. We acknowledge the assistance of the nurse practitioners C. Elton-Lacasse, K. Deemer and R. Ralph as well as the healthcare teams from the Calgary adult ICUs at South Health Campus, Rockyview General Hospital, Foothills Medical Center and Peter Lougheed Center. We thank Haiyan Hou for cytokine analysis and Daniel Young and Laurent Brechenmacher for sample preparation and mass spectrometry. We thank K. Fiest and the ICU study coordinators C. Codan, Z. Slavikova and O. Dmitrieva. We acknowledge D. Jones, C. Curr and the eCritical team (Alberta Health Services) for their help in data acquisition and extraction via eCritical databases. Mortality predictions using dexamethasone-suppressed gene signatures were completed by repurposing computational workflows that were kindly shared by A. Wilk and C. Blish (Stanford University).

\section{Author contributions}

S.S. performed scRNA-seq analyses, prepared figures and co-wrote the paper. N.L.R contributed to experimental design, performed scRNA-seq experiments, prepared figures and co-wrote the paper. A.J., R.A. and L.C. performed bioinformatics and created the online atlas. E.L., R.F. and A.P.N. contributed to sample preparation and scRNA-seq processing. M.G. and B.M. contributed to patient consent and sample collection. L.G.A. and A.D. conducted proteomics and related analyses. M.J.F. provided serum cytokine assays. J.B. and B.Y. conceived of, designed and supervised all experiments and wrote the paper.

\section{Competing interests}

S.S. declares stock ownership in 10x Genomics. All other authors declare no conflicts of interest.

\section{Additional information}

Extended data is available for this paper at https://doi.org/10.1038/s41591-021-01576-3.

Supplementary information The online version contains supplementary material available at https://doi.org/10.1038/s41591-021-01576-3.

Correspondence and requests for materials should be addressed to Nicole L. Rosin, Bryan G. Yipp or Jeff Biernaskie.

Peer review information Nature Medicine thanks Michael Matthay and the other, anonymous, reviewer(s) for their contribution to the peer review of this work. Joao Monteiro was the primary editor on this article and managed its editorial process and peer review in collaboration with the rest of the editorial team.

Reprints and permissions information is available at www.nature.com/reprints. 


\section{ICU Patients}

Assessed for eligibility:

Admission to ICU for respiratory failure with hypoxemia Bilateral infiltrates on chest imaging

Age $\geq 18$ years

PCR (+) for COVID-19 cohort
PCR (-) COVID-19 controls

- Ability to consent or appropriate surrogate

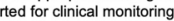
Consecutively enrolled
COVID-19 cohort retrospectively grouped for
analysis by dexamethasone therapy
Exclusions:

Not meeting inclusion criteria Declined to participate or no surrogate Autoimmune disease Immunotherapeutic use in community Active malignancy

\begin{tabular}{|c|c|c|}
\hline$\downarrow$ & $\downarrow$ & $\downarrow$ \\
\hline 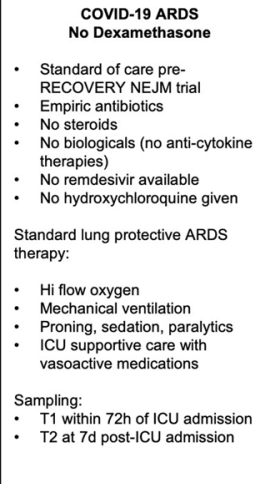 & 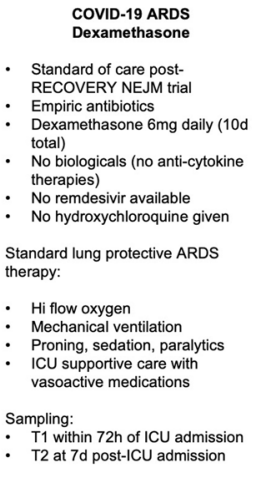 & $\begin{array}{l}\text { Bacterial ARDS } \\
\text { (S. aureus or S. pneumoniae) } \\
: \text { CoVID-19 PCR (-) } \\
: \text { Positive bacterial microbiology } \\
\text { - Standard of care for bacterial } \\
\text { sepsis } \\
: \text { Initial broad spectrum antibiotics } \\
: \text { Vasoactive medications } \\
: \quad \text { No steroids } \\
\text { No biologicals (no anti-cytokine } \\
\text { therapies) } \\
: \quad \text { No remdesivir } \\
\text { Standard lung protective ARDS } \\
\text { therapy: } \\
: \quad \text { Hi flow oxygen } \\
: \quad \text { Mechanical ventilation } \\
: \quad \text { Proning, sedation, paralytics } \\
\text { ICU supportive care } \\
\text { Sampling: } \\
\text { : T1 within } 72 h \text { of ICU admission } \\
\text { T2 at } 7 d \text { post-ICU admission }\end{array}$ \\
\hline
\end{tabular}

Healthy donors

Response to a community based advertisement

Assessed for eligibility.

Age $\geq 18$ years

Asymptomatic
Able to provide consent

Exclusions:

Immunotherapeutic medications Medical history of autoimm
diseases, or malignancy

Extended Data Fig. 1 | A modified CONsolidated Standards Of Reporting Trials (CONSORT) diagram showing trial groups in this study. 


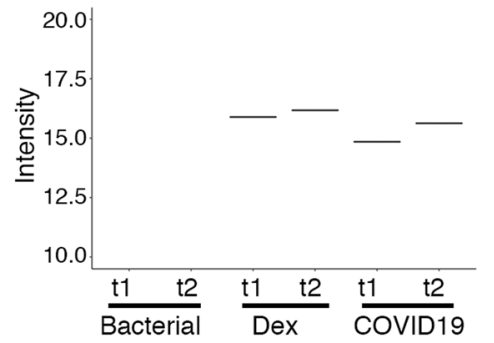

SARS-CoV-2 R1AB

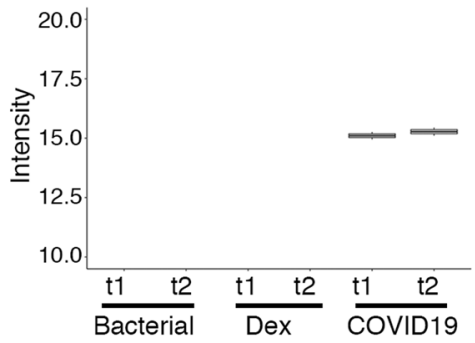

SARS-CoV NS3B

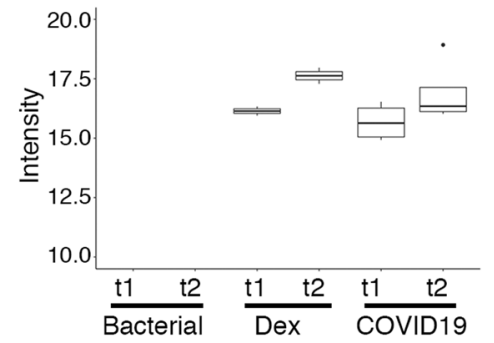

b

ICU Admitted Critically III
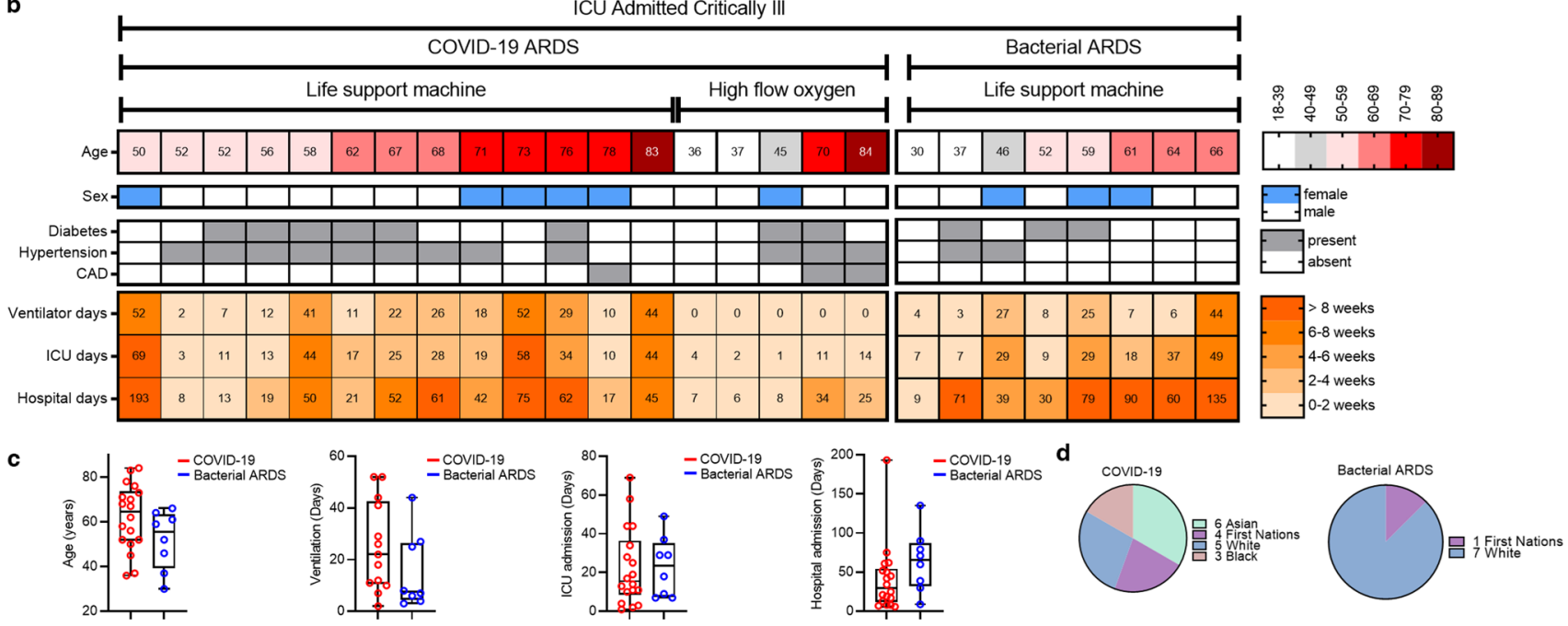

e
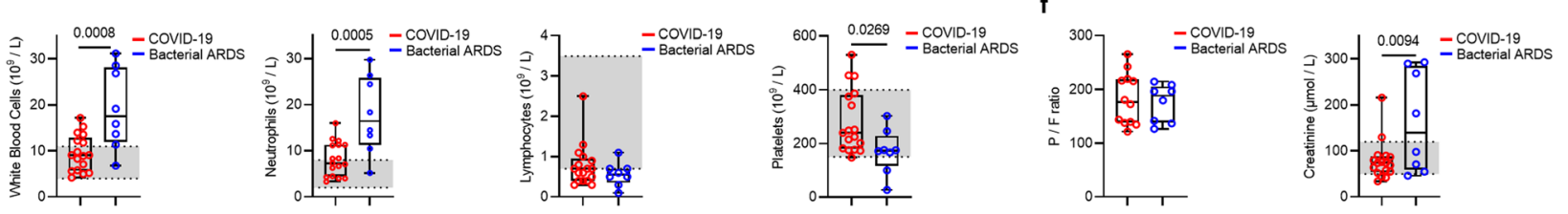

g
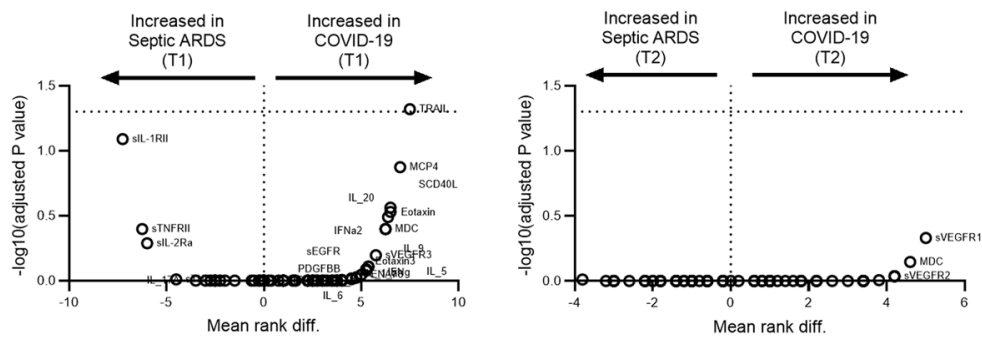

h

Prototypical Cytokine Storm

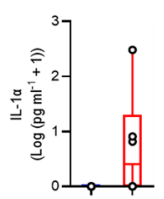

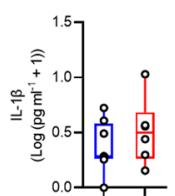
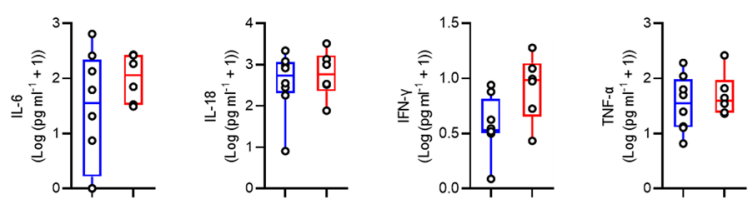

$\square$ Bacterial ARDS

COVID-19 ARDS

i Prototypical Cytokine Release Syndrome
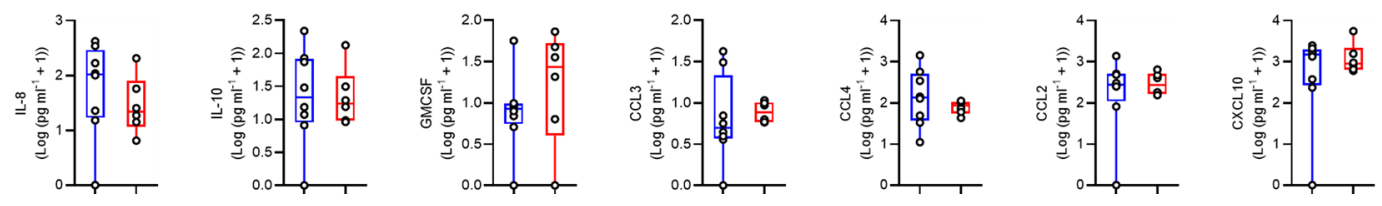

Extended Data Fig. 2 | See next page for caption. 


\section{NATURE MEDICINE}

Extended Data Fig. 2 | Clinical data of ICU admitted COVID-19 and bacterial ARDS. Shotgun proteomics assessment using tandem Mass Spectroscopy with a targeted search run for known SARS-CoV-2 proteins R1A and R1AB, and SARS-CoV protein NS3B are displayed for all patient cohorts (COVID-19 non-dexamethasone $=9$; bacterial ARDS controls $=6$ ). $\mathbf{b}$. Summary of individual information of ICU admitted patients with established COVID-19 or a diagnosis of bacterial ARDS due to sepsis (bacterial ARDS $n=5$ at $t 1, n=4$ at t2; COVID-19 ARDS $n=8$ at $t 1, n=4$ at t2). Age, sex, comorbidities and lengths of stay are displayed. Life support machine includes mechanical ventilation (and ECMO in the instance of sample C3 at t2). c-d. Aggregated cohort clinical data (c) and racial backgrounds (d). e. Clinical cell counts from peripheral blood taken on t1; shaded areas show local lab normal values. f. PaO2/ $\mathrm{FiO} 2$ ratio (P/F) and creatinine at $\mathrm{t}$. g. Multiple comparison analysis of all serum cytokines assessed at $\mathrm{t} 1$ and $\mathrm{t} 2$ are shown as volcano plots. Significance was estimated using two-tailed Mann-Whitney $U$ test with Holm-Sidak multiple-testing correction. (h-i) Serum cytokine determination of prototypical mediators involved in (h) cytokine storm and (i) cytokine release syndrome graphed in Log transformation taken at t1. Box plots include a line across the box, upper hinge, and lower hinge which represent median, 75th percentile (Q3), and 25th percentile (Q1), respectively. The lower and upper hinges correspond to the first and third quartiles. The upper whisker extends from the hinge to the largest value no further than $\mathrm{Q} 3+1.5 \times$ interquartile range (IQR). The lower whisker extends from the hinge to the smallest value at most Q1 - 1.5 * IQR. 


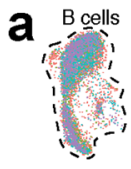

Monocytes

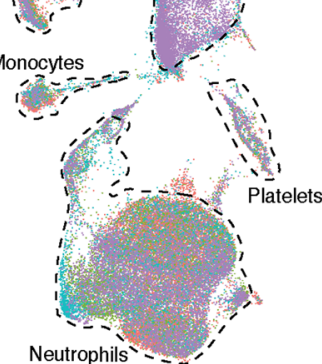

a

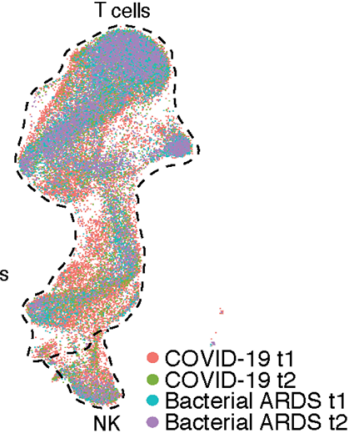

b

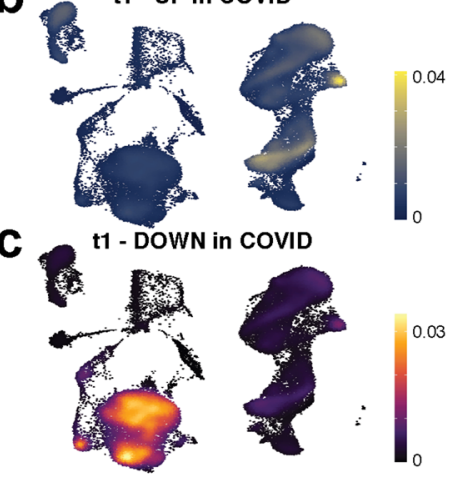

d

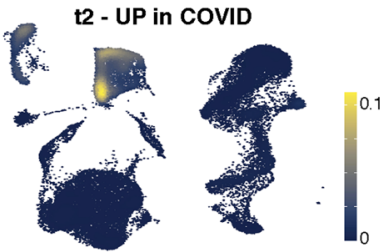

e

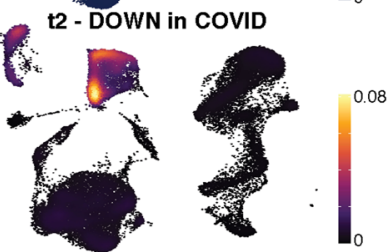

f
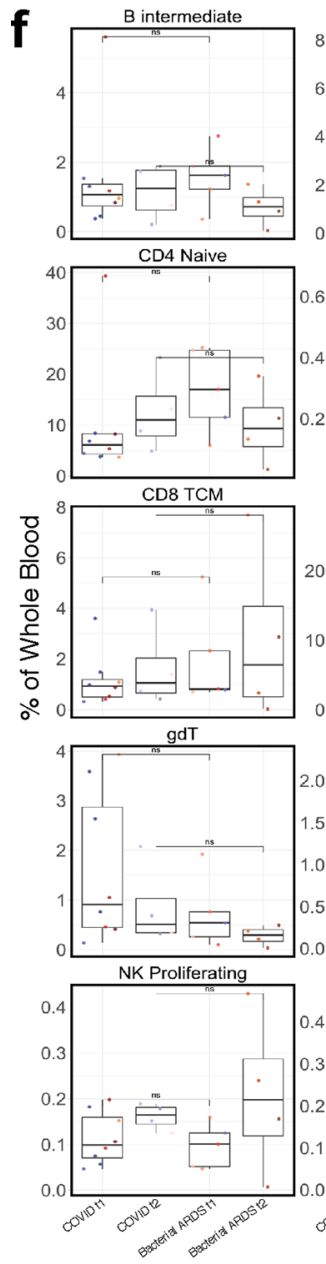

g

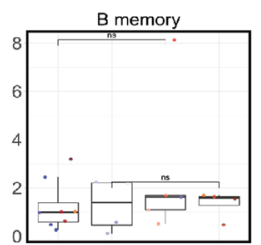

CD4 Proliferating
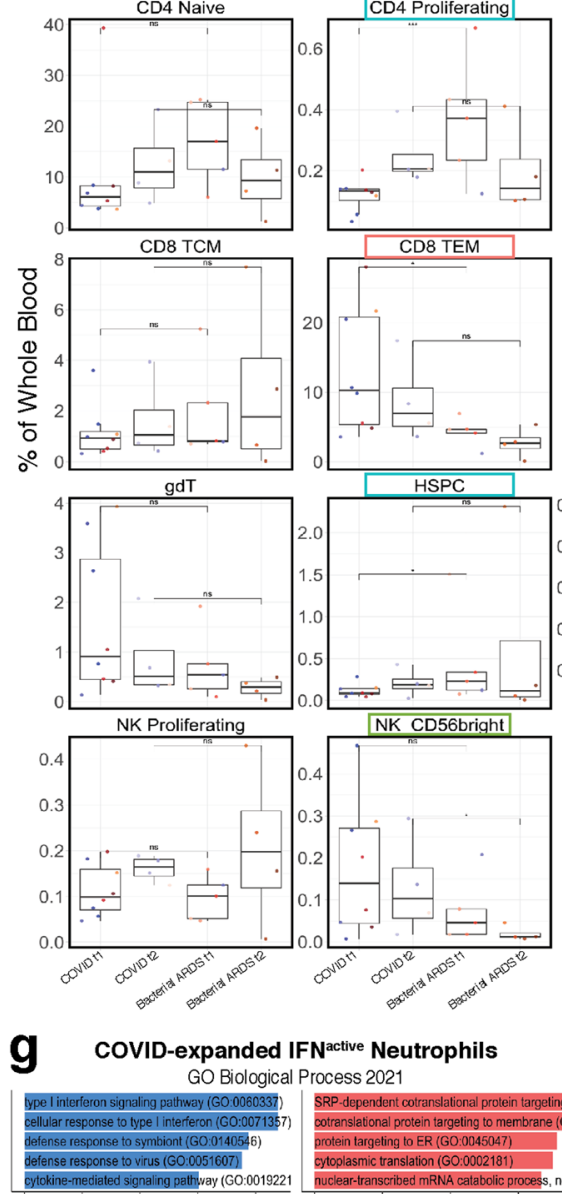

GO Cellular Component 202

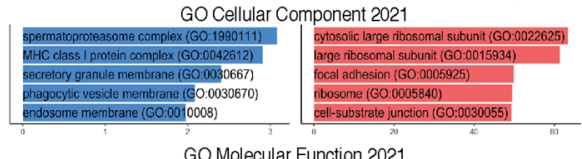

GO Molecular Function 2021

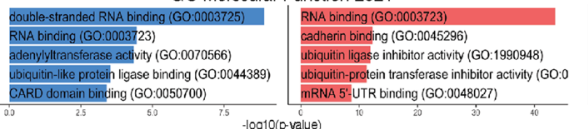

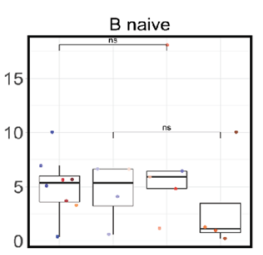
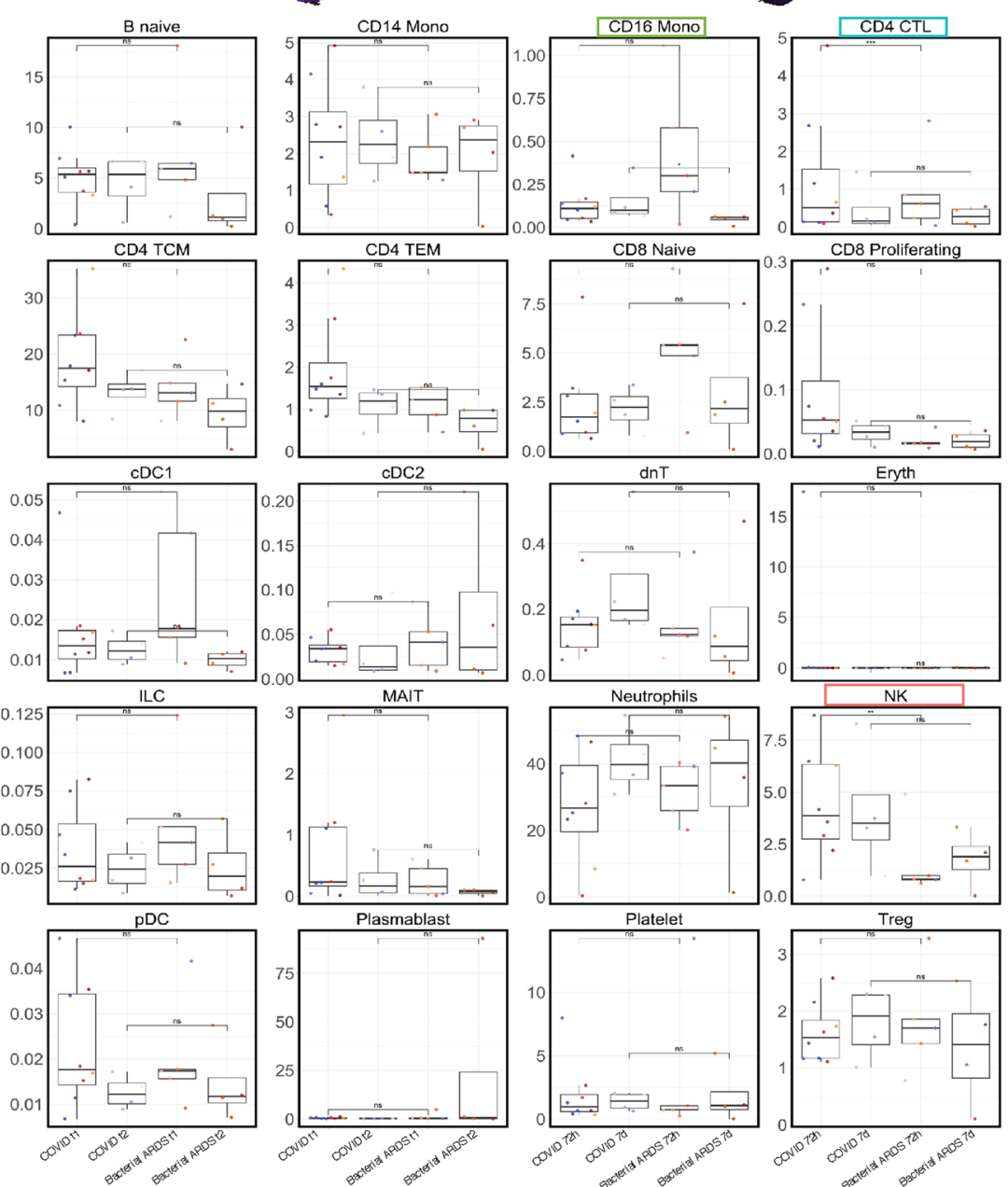

h coviD-expanded PGactive Neutrophils
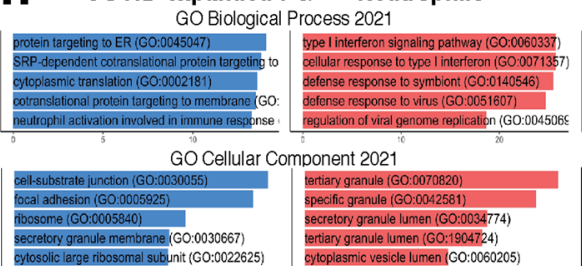

GO Molecular Function 2021

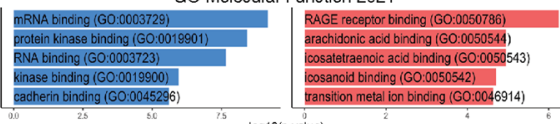

Et $\quad$ log10(p-value)
$\square$ Positive regulation $\square$ Negative regulation
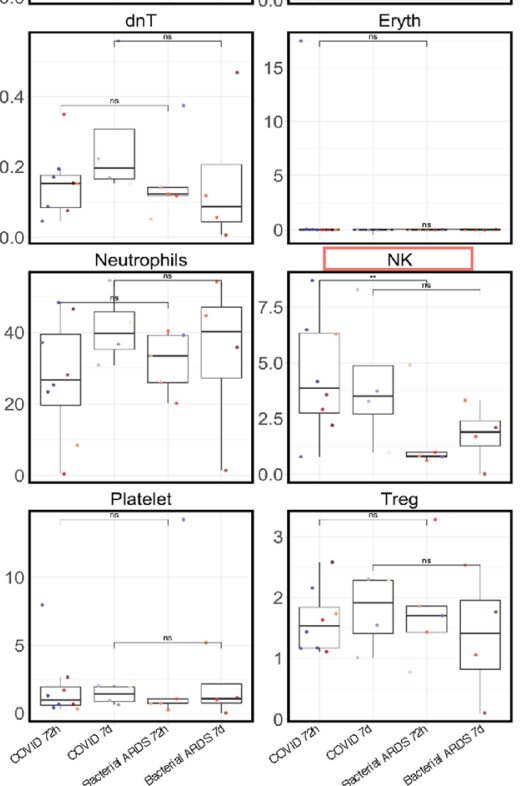
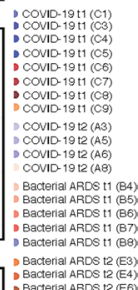

Extended Data Fig. 3 | See next page for caption. 


\section{NATURE MEDICINE}

Extended Data Fig. 3 | COVID-19 elicits distinct innate and adaptive immune responses compared to bacterial ARDS. a. UMAP projection of 86,935 whole blood cells from 21 patient samples, coloured by clinical cohort. b-e. Kernel density estimates depicting magnitude of response elicited by immune cell subsets in COVID-19 t1 (b-c) and t2 (d-e) calculated by summing consensus DEG fold changes for each cell subset shown in Panel B. Consensus DEGs upregulated in COVID-19 are plotted on cividis spectrum (yellow = higher expression) whereas downregulated DEGs are plotted on inferno spectrum (yellow/orange = lower expression). f. Boxplots showing percentage of each cell type in each patient sample grouped by clinical cohort and coloured by donor ID. The $x$ axes correspond to the clinical cohort of each patient. Biologically independent samples for COVID-19 at t1 $(n=8)$, COVID-19 at t2 $(n=4)$, bacterial ARDS at $\mathrm{t} 1(n=5)$, and bacterial ARDS at $\mathrm{t} 2(n=4)$. Significance of effects was estimated using two-sided generalized linear mixed-effects model ( $g$ Imer, binomial distribution) with fixed (COVID-19 vs Bacterial ARDS) and random (per patient) effects. P-values were calculated using likelihood-ratio chi-square for generalized linear models (using R package 'car') and were adjusted for multiple comparisons using Bonferroni correction. All effects with $p<0.05$ are indicated. ${ }^{\star} p<0.05,{ }^{\star \star} p<0.01,{ }^{\star \star \star} p<0.001$. Absolute $p$-adjusted values are provided in Supplementary Table 8. g-i. Gene Ontology (GO) enrichment depicting top five positively (blue) and negatively (red) regulated terms across all three domains (cellular component, biological process, and molecular function) describing biological activity of gene signatures for IFNactive (g), PGactive (h), and bacterial-expanded (i) neutrophil states. 

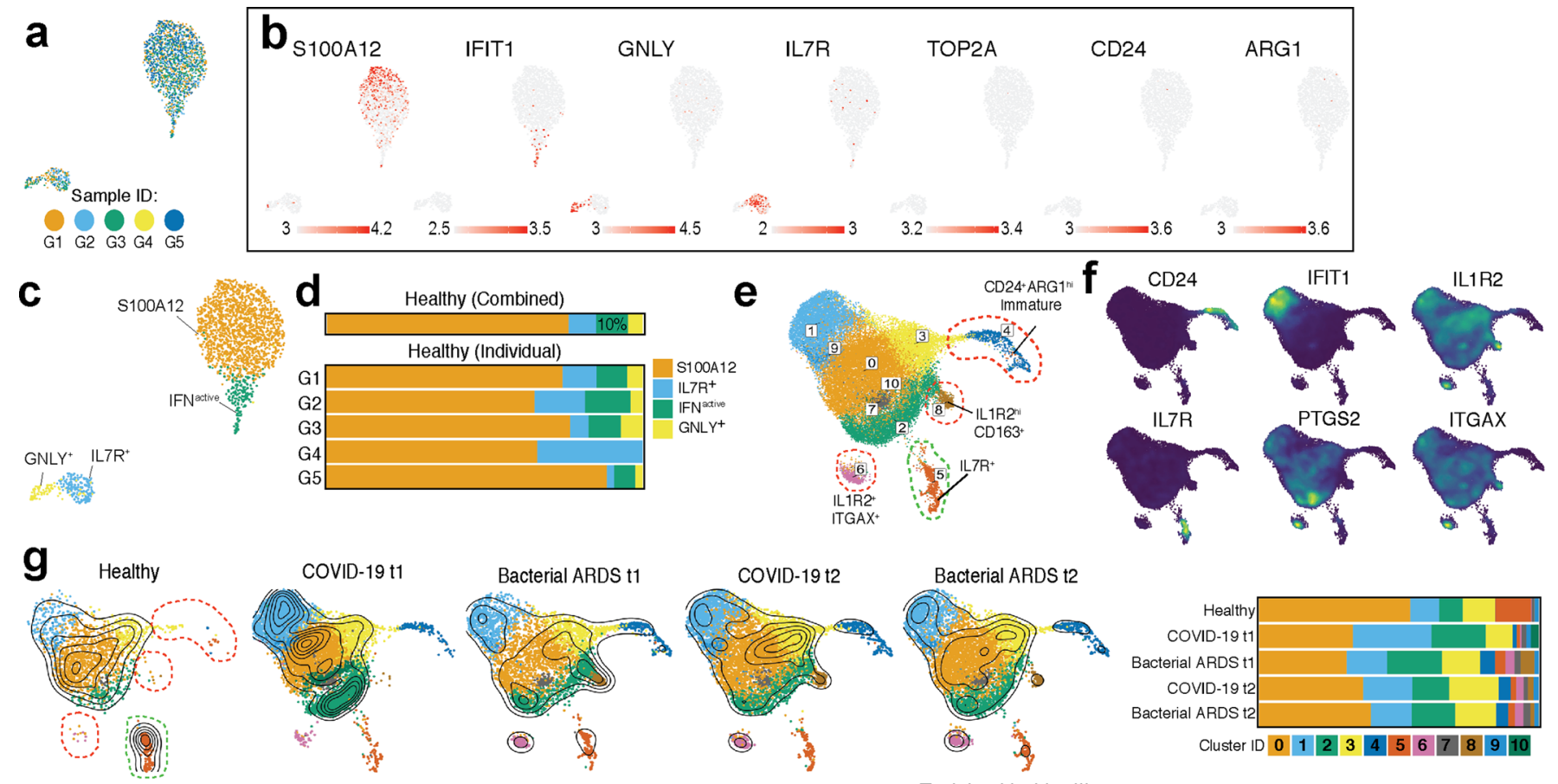

...-. Absent in Healthy ....-Enriched in Healthy

Cluster ID \begin{tabular}{ll|ll|l|l|l|l|l|l|l|}
0 & 1 & 2 & 3 & 4 & 5 & 6 & 7 & 8 & 9 & 10
\end{tabular}

IFNactive Neutrophils Subclustered
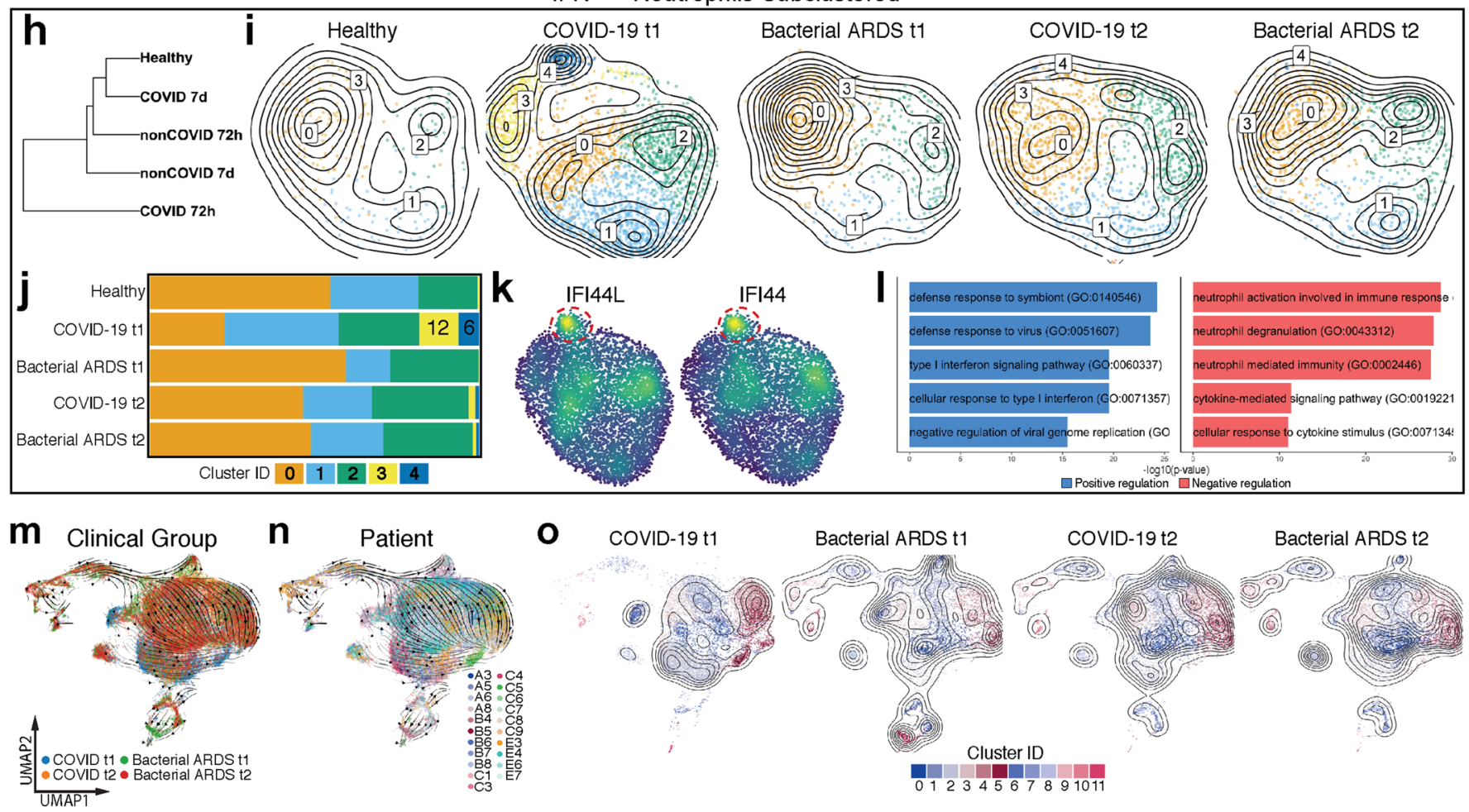

Bacterial ARDS t2

p Velocity Length $\mathbf{q}$ Velocity Vector Field $\mathbf{r}$ TOP2A Expression $\mathbf{S}$
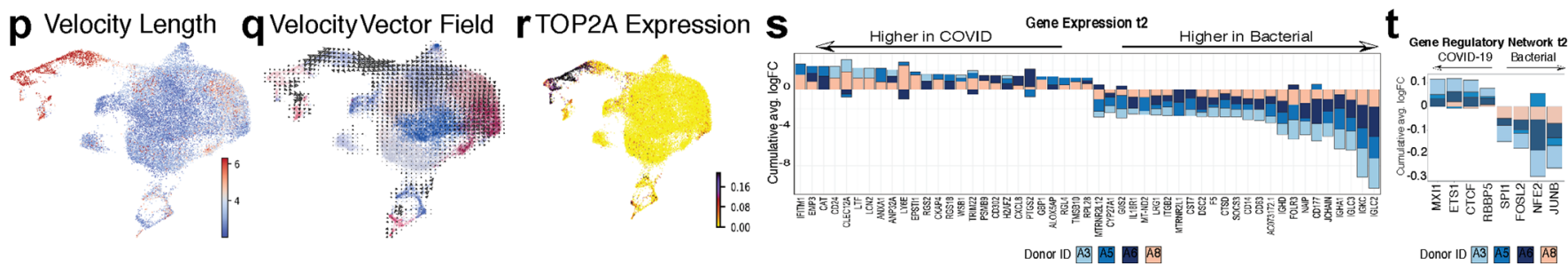

Extended Data Fig. 4 | See next page for caption. 


\section{NATURE MEDICINE}

Extended Data Fig. 4 | COVID-19 infection reprograms neutrophil maturation by driving expansion of ISG- and PG-expressing subsets. a. UMAP of neutrophils from healthy donors ( $n=1,912$ cells) colored by donor of origin. $\mathbf{b}$. Neutrophil state-defining markers. c. UMAP of neutrophils from healthy donors colored by neutrophil states. d. Neutrophil state composition in healthy donors, combined across all donors or separated by individual donor ID. e. Subclustered neutrophils, integrated across patient cohorts to compare healthy, bacterial ARDS at $\mathrm{t} 1$ and t2, and COVID-19 ARDS at t1 and t2. f. Kernel density plots showing expression of neutrophil state-defining markers. g. Cohort-separated UMAPs, colored by subcluster ID and overlaid with cell density contour plots, and bar plot depicting neutrophil cluster composition across cohorts. h. Dendrogram showing unsupervised hierarchical clustering of IFNactive neutrophils by showing relatedness of this subset across patient cohorts. $\mathbf{i}$-j. Cohort-separated UMAPs of IFNactive neutrophils, colored by subcluster ID and overlaid with cell density contour plots ( $\mathrm{i}$ ), and bar plot showing cluster composition ( $\mathrm{j}$ ) across cohorts. k-I. Expression of genes ( $\mathrm{k}$ ) and signalling pathways (I) enriched in COVID-19 t1 IFNactive neutrophil relative to remaining cohorts. m-n. UMAP plotting velocity analysis of 29,653 subclustered neutrophils undergoing state transitions, coloured by clinical cohort $(m)$ and donor IDs $(n)$. $\mathbf{o}$. Cohort-separated UMAPs, colored by neutrophil subcluster ID and overlaid with cell density contour plots. p-q. Subclustered neutrophil UMAPs, coloured by magnitude of velocity vector length reflecting the difference between expected versus recovered unspliced counts $(p)$ and neutrophil Louvain clusters overlaid with velocity vector fields ( $q$ ). $\mathbf{r}$. Expression of immature neutrophil marker TOP2A. s-t. Consensus plot of differentially expressed genes ( $\mathrm{s}$ ) and SCENIC-inferred transcription factors ( $\mathrm{t}$ ) upregulated (positive $\log F C$ ) or suppressed (negative $\log F C$ ) in neutrophils from at least 2 of 4 patients with COVID-19 relative to bacterial ARDS at t2. 
a

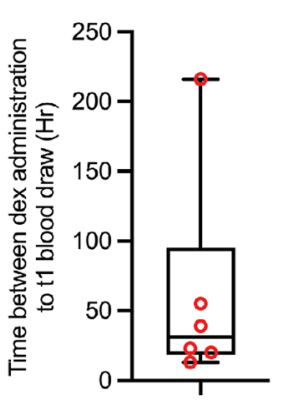

b

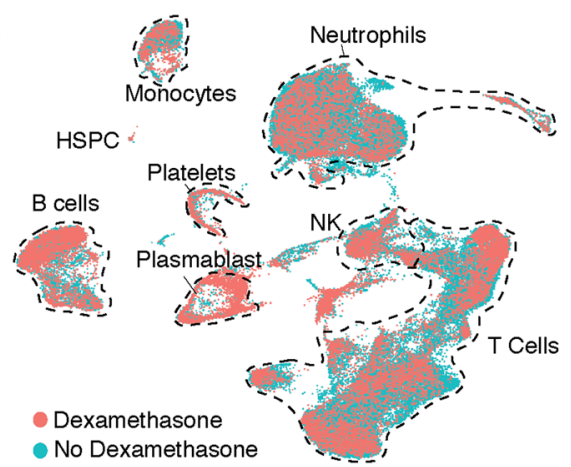

C

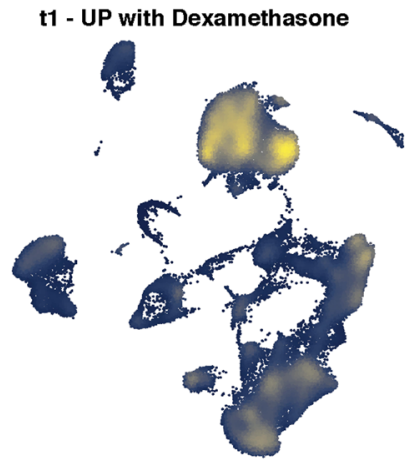

e 11 - Down with Dexamethasone

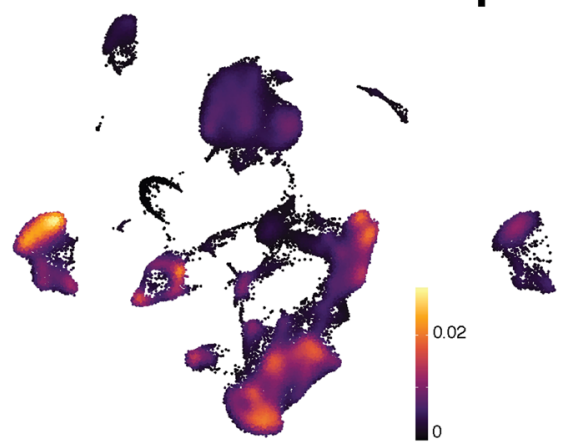

d 12 - UP with Dexamethasone

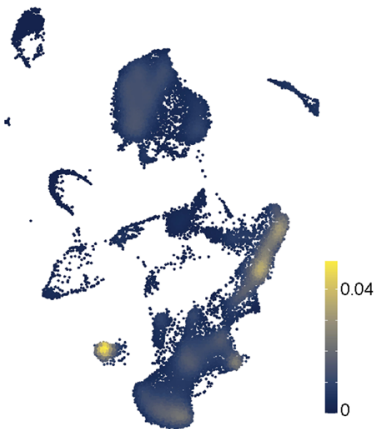

f 12 - Down with Dexamethasone

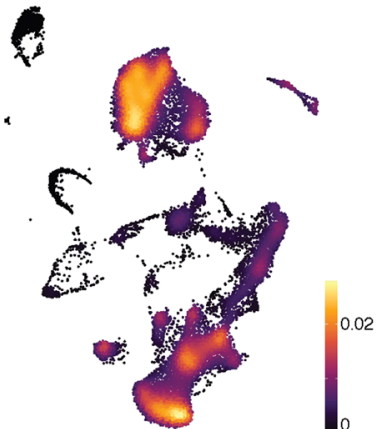

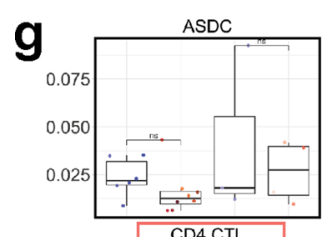
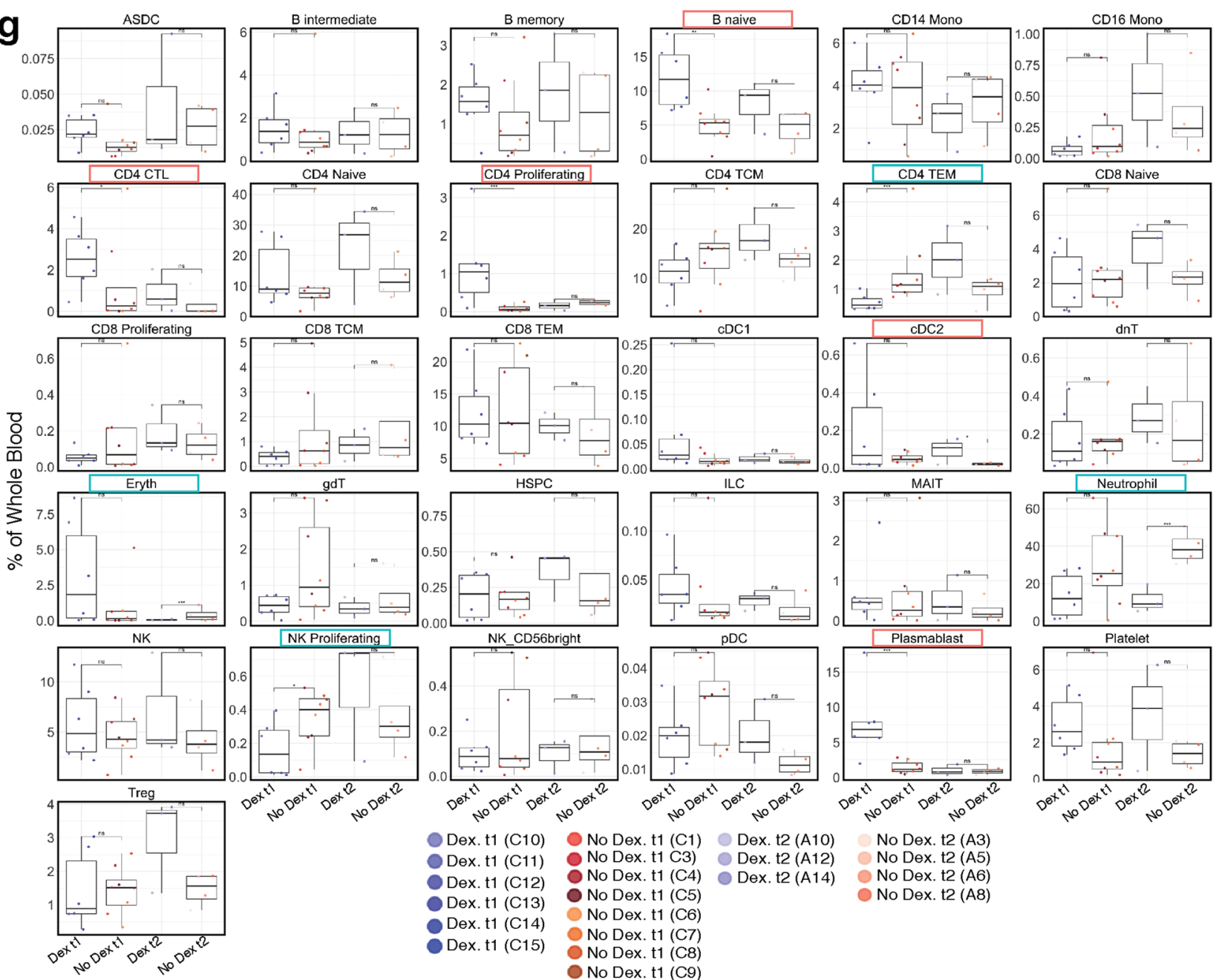

Dex.t1 (C10) No Dex.t1 (C1) Dex. t2 (A10)

Dex. t1 (C11) No Dex. t1 C3) Dex. t2 (A12)

Dex. 11 (C12) $O$ No Dex. 11 (C4)

No Dex.t1 (C6)

Dex. 1 ( (C14) No Dex. t1 (C7)

Dex. 11 (C15) No Dex. t1 (C7)

No Dex. 11 (C9)

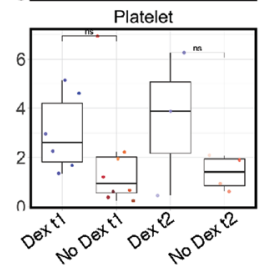

Extended Data Fig. 5 | See next page for caption. 


\section{NATURE MEDICINE}

Extended Data Fig. 5 | Immunosuppressive effects of dexamethasone are mediated through neutrophils and multiple adaptive immune cell subsets. a. Bar plot shows distribution of time interval (in hours) between dexamethasone administration to first blood draw at t1. Box plot include a line across the box, upper hinge, and lower hinge which represent median, Q3, Q1, respectively. The lower and upper hinges correspond to the Q1 and Q3. The upper whisker extends from the hinge to the largest value no further than $Q 3+1.5 \times$ interquartile range (IQR). The lower whisker extends from the hinge to the smallest value at most Q1 - 1.5 * IQR. b. UMAP projection of 80,994 whole blood cells from 21 patient samples, coloured by treatment groups (nondexamethasone COVID-19 ARDS ( $n=8$ at $t 1, n=4$ at t2; dexamethasone-treated COVID-19 ARDS $(n=6$ at $t 1, n=3$ at t2)) c-f. Kernel density estimates depicting magnitude of response elicited by immune cell subsets following dexamethasone treatment at 72 hours post-ICU (c-d) and 7 days post-ICU (e-f) calculated by summing consensus DEG fold changes for each cell subset shown in Panel B. Consensus DEGs upregulated following dexamethasone treatment are plotted on cividis spectrum whereas downregulated DEGs are plotted on inferno spectrum. $\mathbf{g}$. Boxplots showing percentage of each cell type in each patient sample grouped by treatment and coloured by donor ID. The $x$ axes correspond to four treatment groups. $n=6, n=8, n=3$, and $n=4$ biologically independent samples from dexamethasone-treated t1, no dexamethasone t1, dexamethasone-treated t2, no dexamethasone t2, respectively. Significance of effects was estimated using two-sided generalized linear mixed-effects model (glmer, binomial distribution) with fixed (dexamethasone vs no dexamethasone) and random (per patient) effects. P-values were calculated using likelihood-ratio chi-square for generalized linear models (using R package 'car') and were adjusted for multiple comparisons using Bonferroni correction. All effects with $p<0.05$ are indicated. ${ }^{\star} p<0.05,{ }^{\star *} p<0.01$, ${ }^{\star \star \star} p<0.001$. Absolute $p$-adjusted values are provided in Supplementary Table 8. 
a

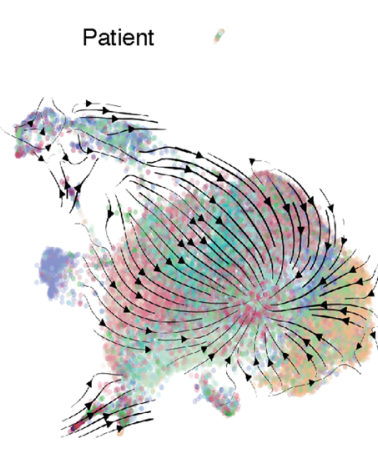

- 0 O O O 00.00 A10 A12 A14 A3 A5 A6 A8 C1 C10 11 C12 C13 C14 C15 C3 C4 C5 C6 C7 C8 C9

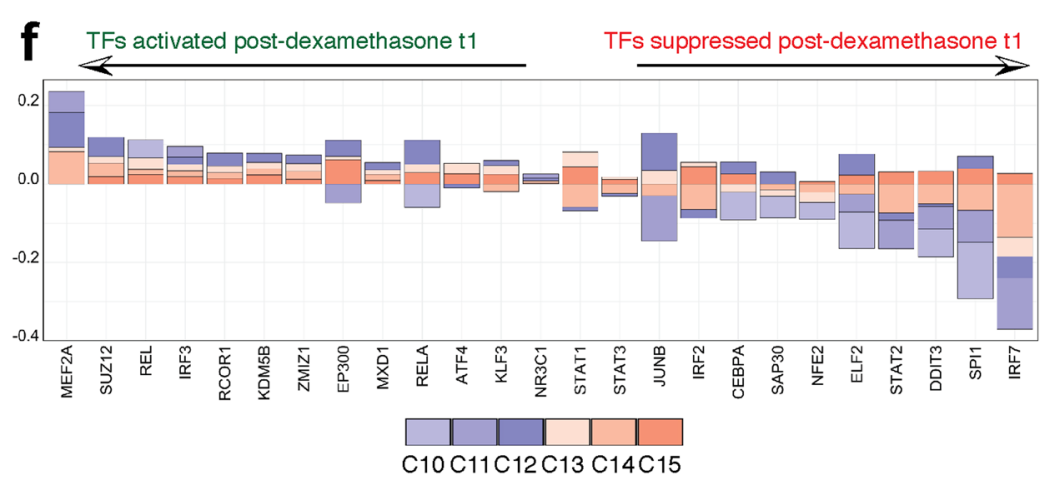

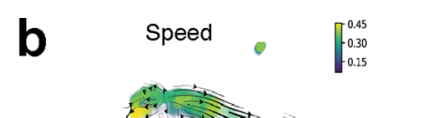

C Acceleration ,

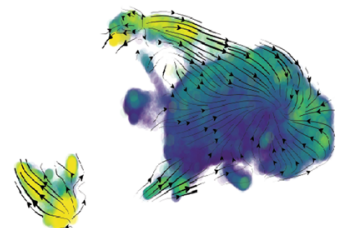

d

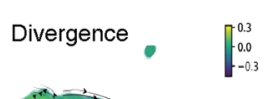

e

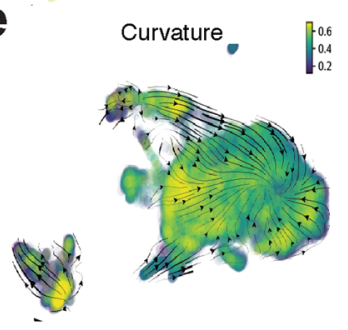

g
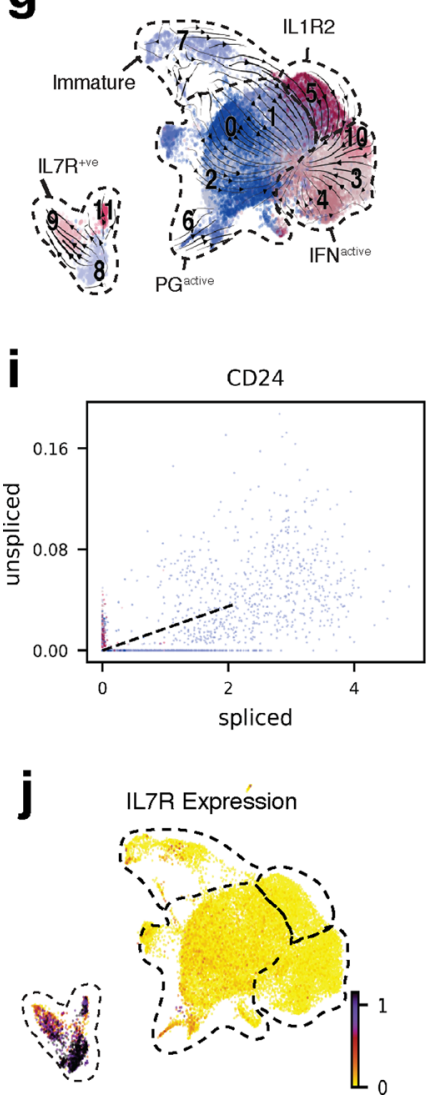
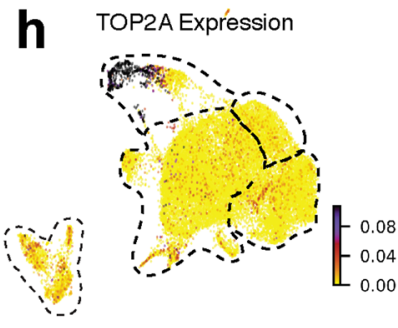

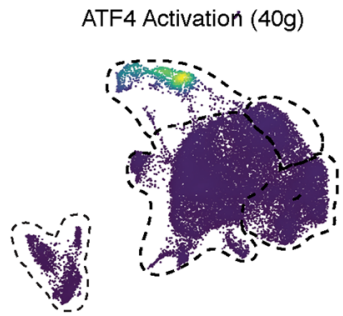

CD24 Expression

CD24 Velocity

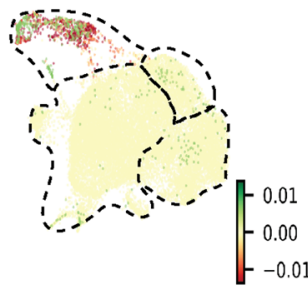

k
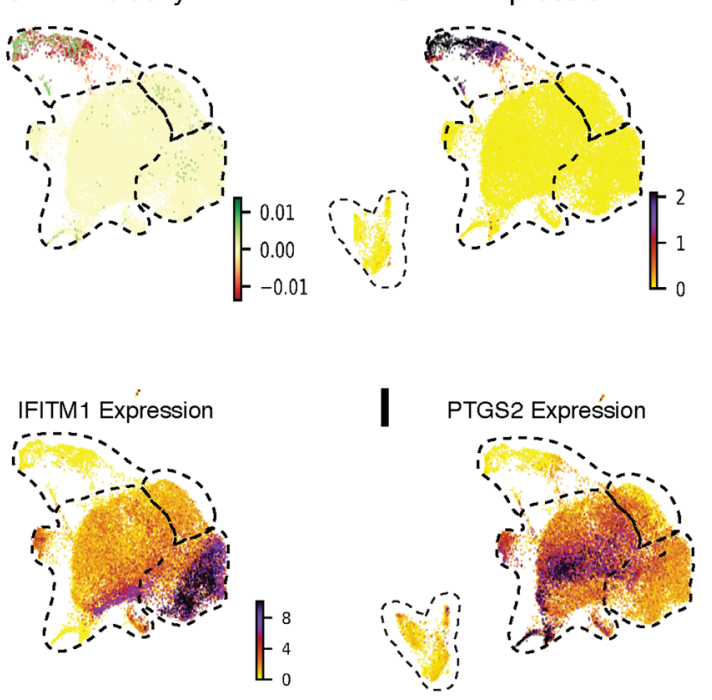

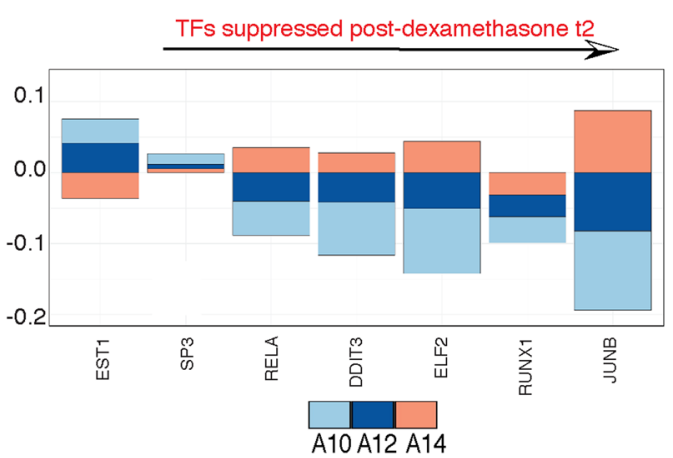

JDP2 Activation (12g)
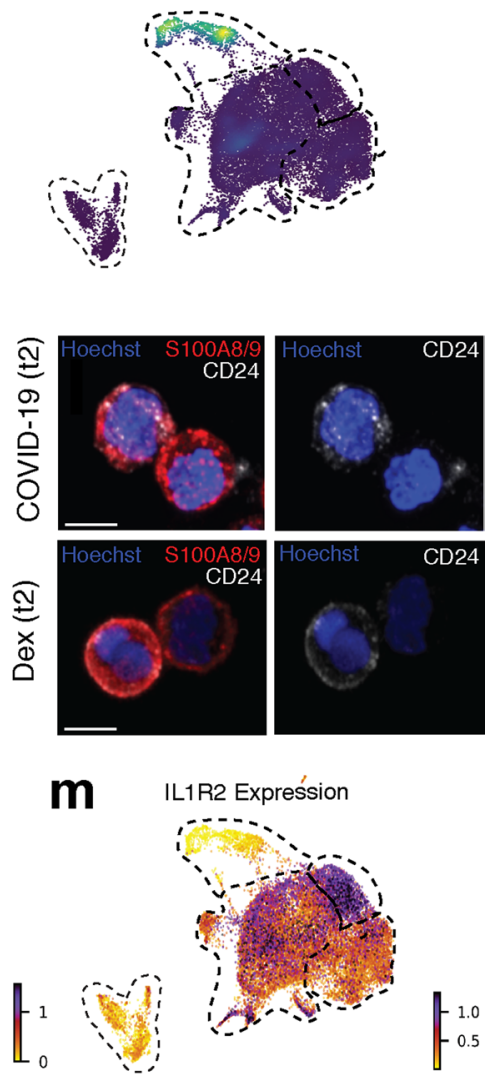
Extended Data Fig. 6 | Distinct neutrophil states and their response to dexamethasone. a. UMAP projection of subclustered neutrophils from 21 patient samples, coloured by individual patient ID. b-e. Cell speed (length of velocity vectors; b), acceleration (subspaces where velocity undergoes dramatic changes in either in magnitude or direction; $\mathbf{c}$ ), divergence (outward flux indicating the extent to which a point behaves like a source; d) and curvature (hotspots of abrupt vector field change; e). f. Differentially activated consensus TFs upregulated (positive logFC) or suppressed (negative logFC) post-dexamethasone in at least 3 of 6 patients at t1, and in at least 2 of 3 patients at t2. g-j. Neutrophil states (g) can be distinguished by expression of proliferative marker TOP2A and activation of immaturity-associated TFs ATF4 and JDP2 (h), CD24 splicing kinetics, velocity, expression and immunocytochemistry (representative images are shown; $n=3$ for each group; Scale bar, $5 \mu \mathrm{m}$ ) (i), IL-7R (j), Interferon-stimulated genes such as IFITM1 (k), genes involved in prostaglandin synthesis such as PTGS2 (I), and IL-1R2 (m). 
a

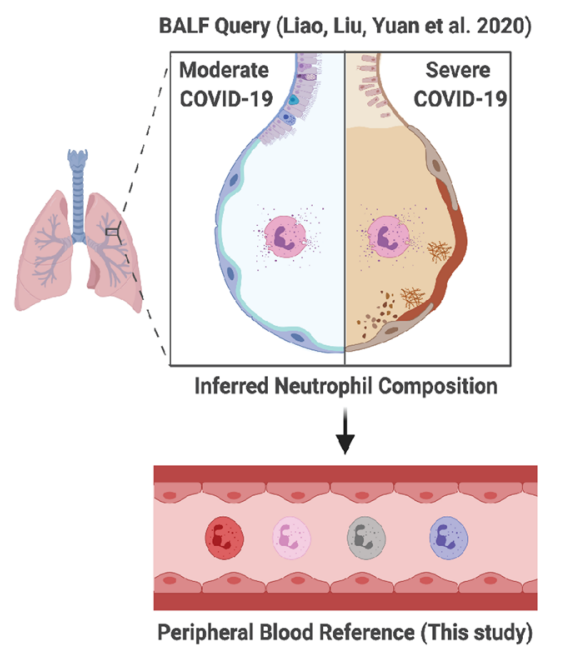

b

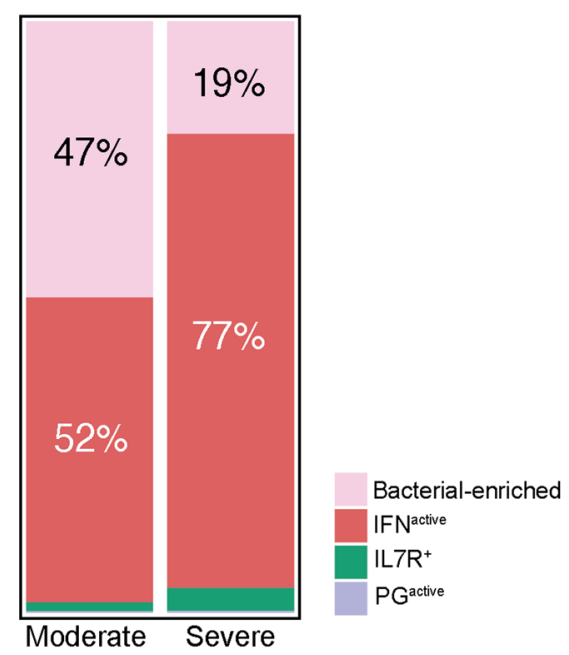

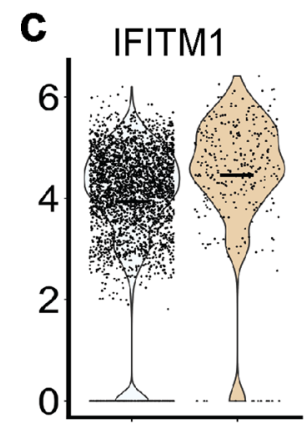

IFITM2

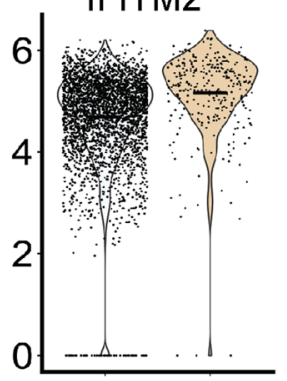

IFI6
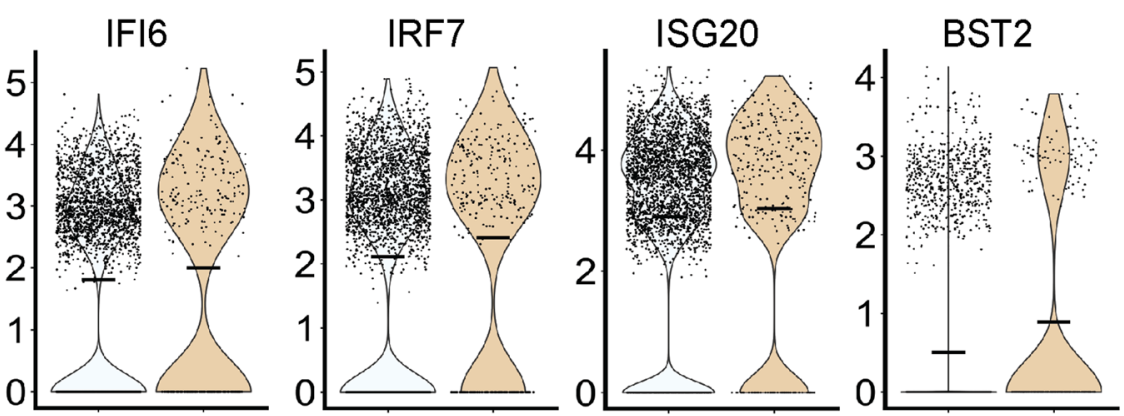

- Moderate COVID-19

d

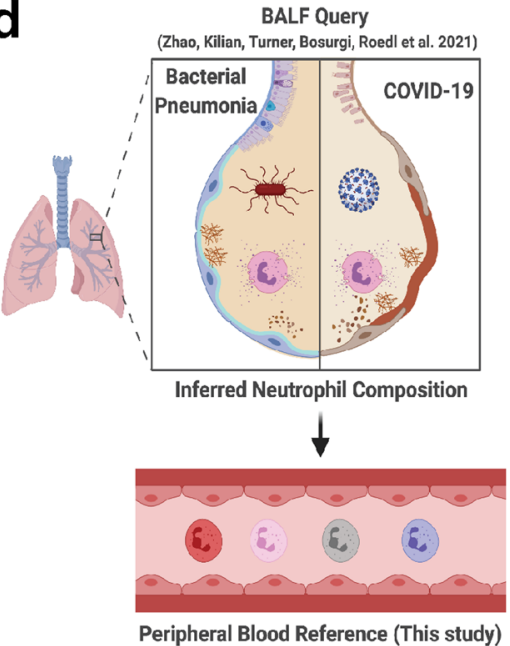

e

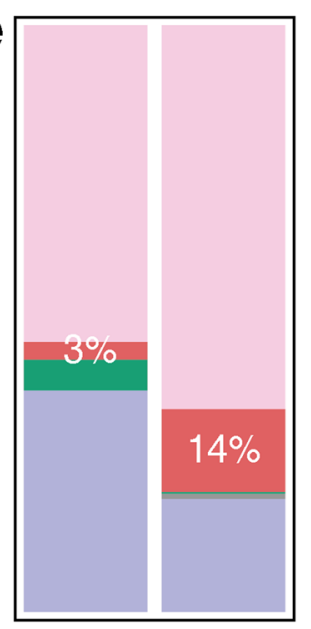

Bacterial COVID19

Pneumonia

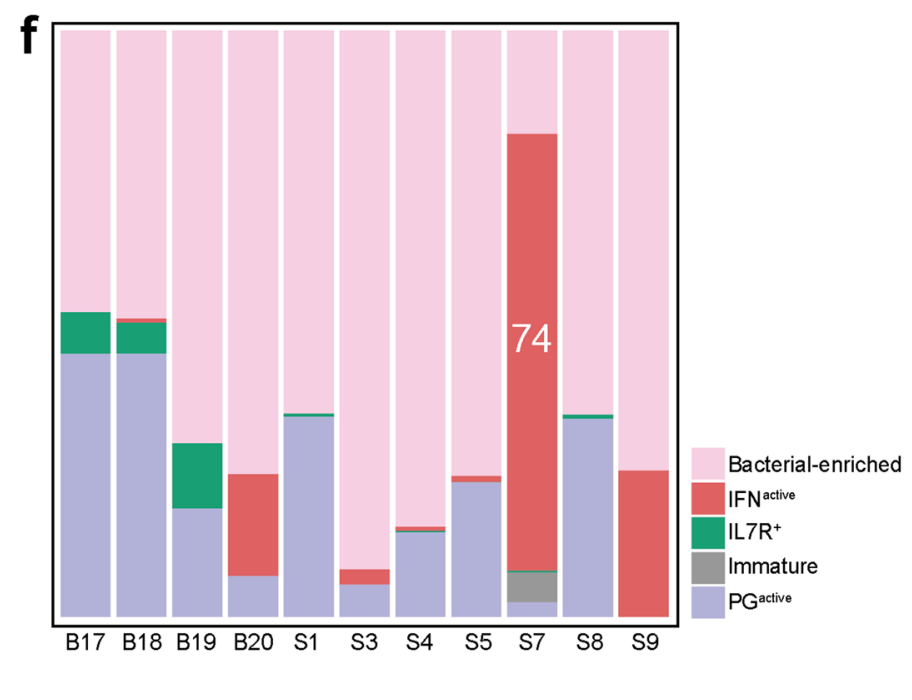

Extended Data Fig. 7 | Inferred neutrophil composition in bronchoalveolar microenvironments. a. Strategy for inferring BALF neutrophil composition in severe and moderate COVID-19 by reference-projecting to neutrophil states in peripheral blood. b. Proportion of neutrophil states in bronchoalveolar microenvironment in severe and moderate COVID-19. c. Expression of Type 1 IFN genes in neutrophils across severe and moderate COVID-19 patients. d. Strategy for inferring BALF neutrophil composition in bacterial pneumonia versus COVID-19. e-f. Proportion of neutrophil states in bronchoalveolar microenvironment, separated by bacterial pneumonia and COVID-19 (e) and individual donors ( $f$ ). 
a

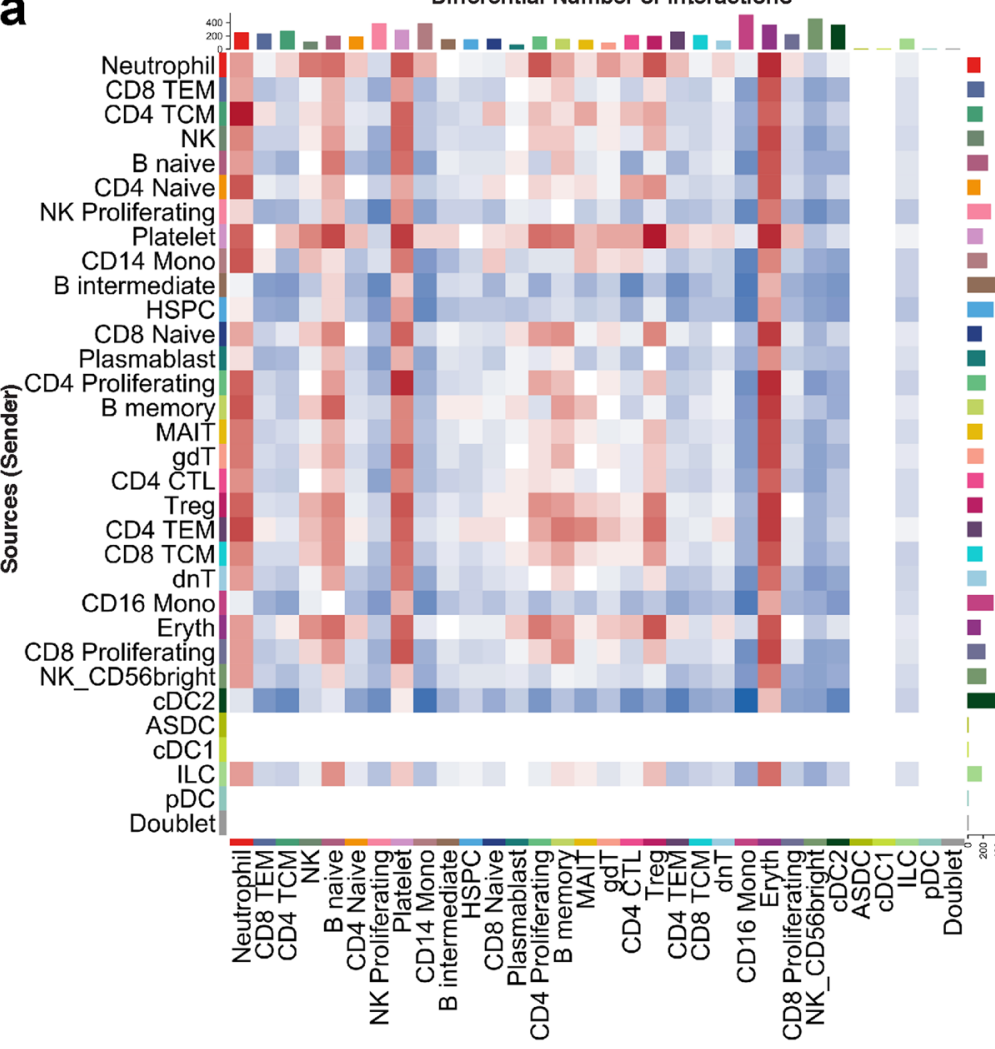

d

Dex Enhanced Signalling
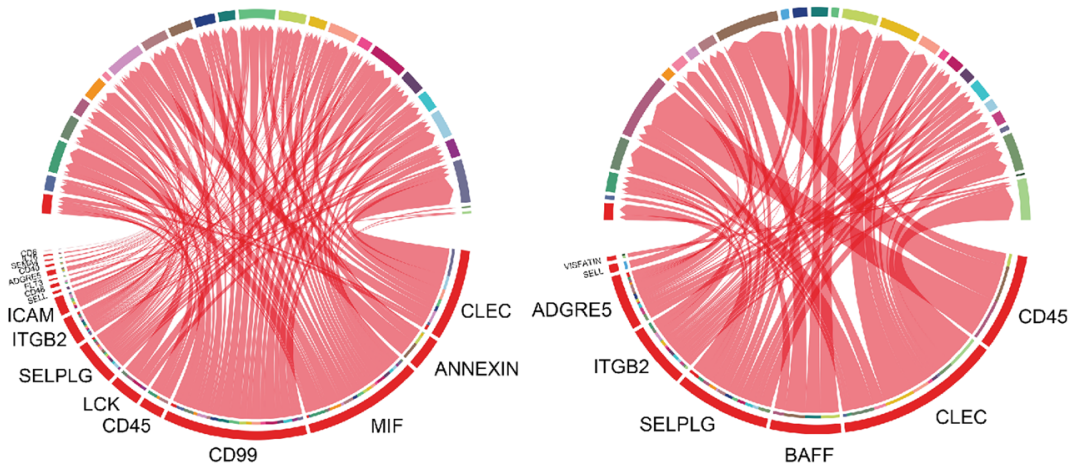

Dex Suppressed Signalling

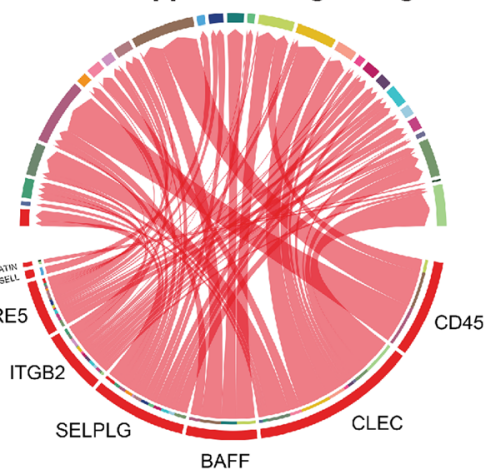

b

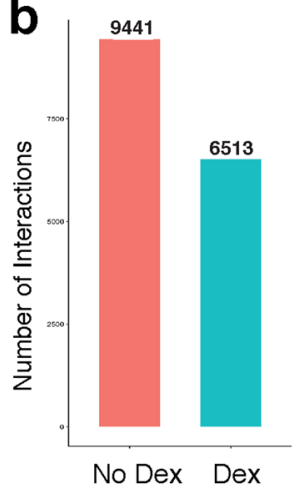

Relative

Values
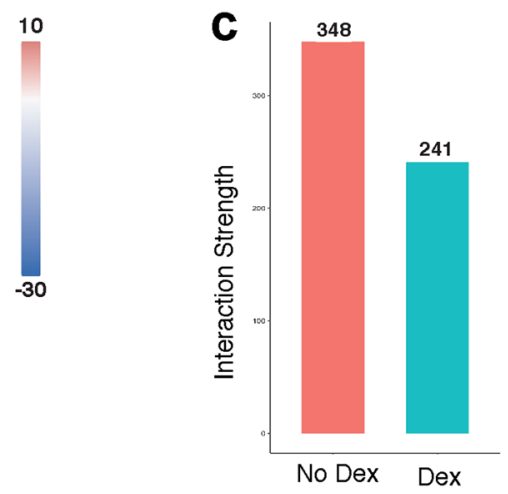

e No Dex (Annexin Family)

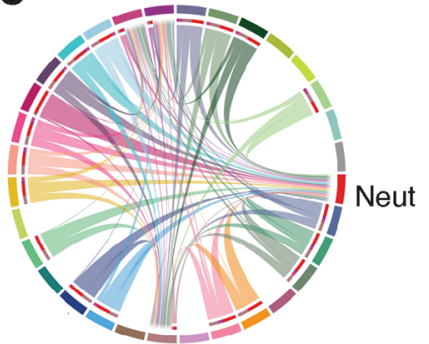

f Dex (Annexin Family)

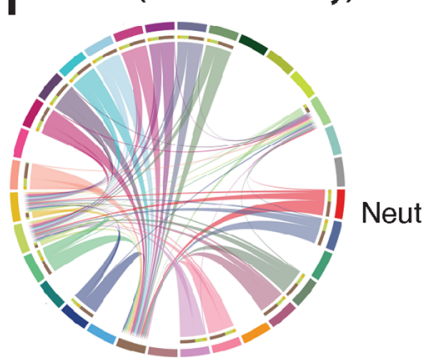

Cell State

Neutrophil

CD8 TEM

CD4 TCM

NK

$B$ naive

CD4 Naive

NK Proliferating

Platelet

CD14 Mono

$B$ intermediate

HSPC

Plasmablas

CD4 Proliferating

B memory

MAIT

gdT

CD4 CTL

Treg

CD4 TEM
CD8 TCM

dnT

CD16 Mono

Eryth

CD8 Proliferating

NK_CD56bright

CDC̄ 2

ASDC

$\mathrm{CDC}$

ILC

$\mathrm{pDC}$

g

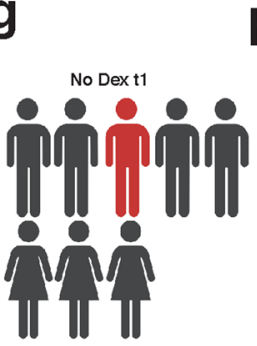

Final Outcome

Alive $\square$ Death
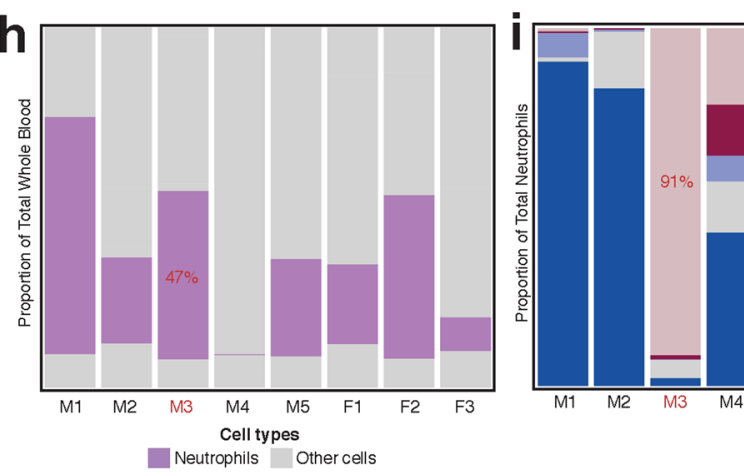
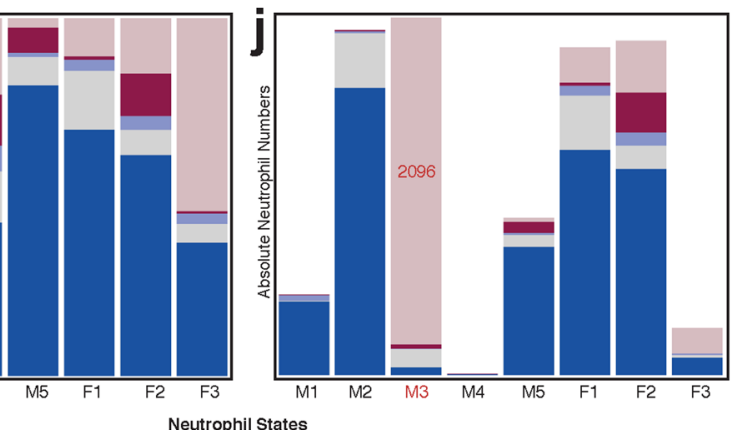

PG active Other $\square$ Immature $\square$ IL1R2 active $\square$ IFN arctwe

Extended Data Fig. 8 | See next page for caption. 
Extended Data Fig. 8 | Dexamethasone alters global signaling topology and increased proportion of IFN ${ }^{\text {active }}$ neutrophils are associated with mortality. a. Interaction heatmap summarizing differential number of incoming (top bar plot) and outgoing (right bar plot) cell-to-cell interactions following dexamethasone treatment. b-c. Global summary of number (b) and strength (c) of all interactions different immune cell types with and without dexamethasone. d. Neutrophil-driven signaling pathways enhanced and supressed with dexamethasone, identified using CellChat (MHC-I signaling filtered out). e-f. Unfiltered topology of annexin signaling without (e) and with dexamethasone (f) treatment. g. Schematic depicting outcomes in nondexamethasone treated COVID-19 patients. Male 3 (M3) succumbed to disease. h-i. Proportion of neutrophils (h) and neutrophil states (i) in whole blood samples from individual donors ( 5 males, 3 females) at $\mathrm{t} 1$. j. Raw neutrophil state counts from the same 8 donors at $\mathrm{t}$. 

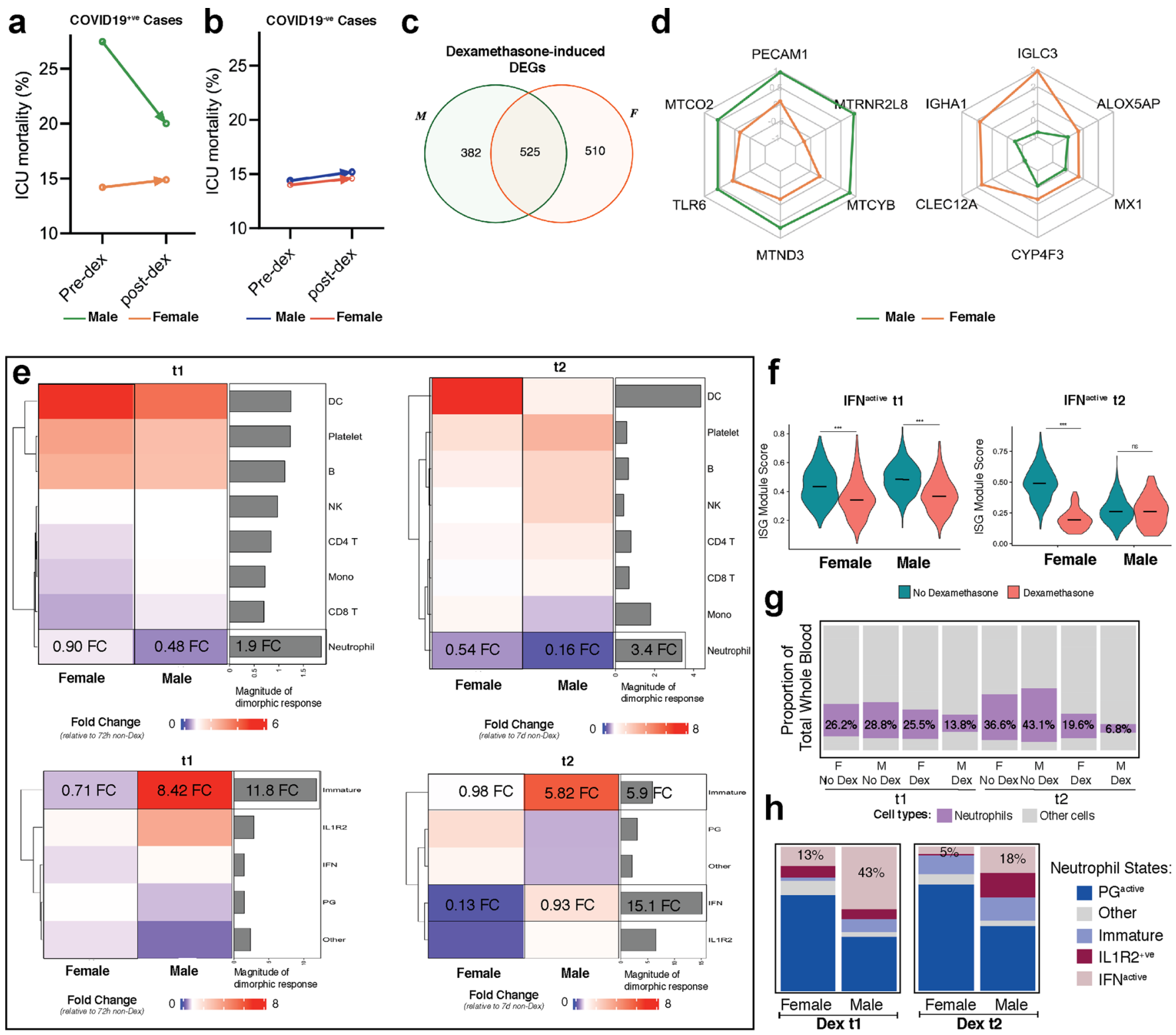

i
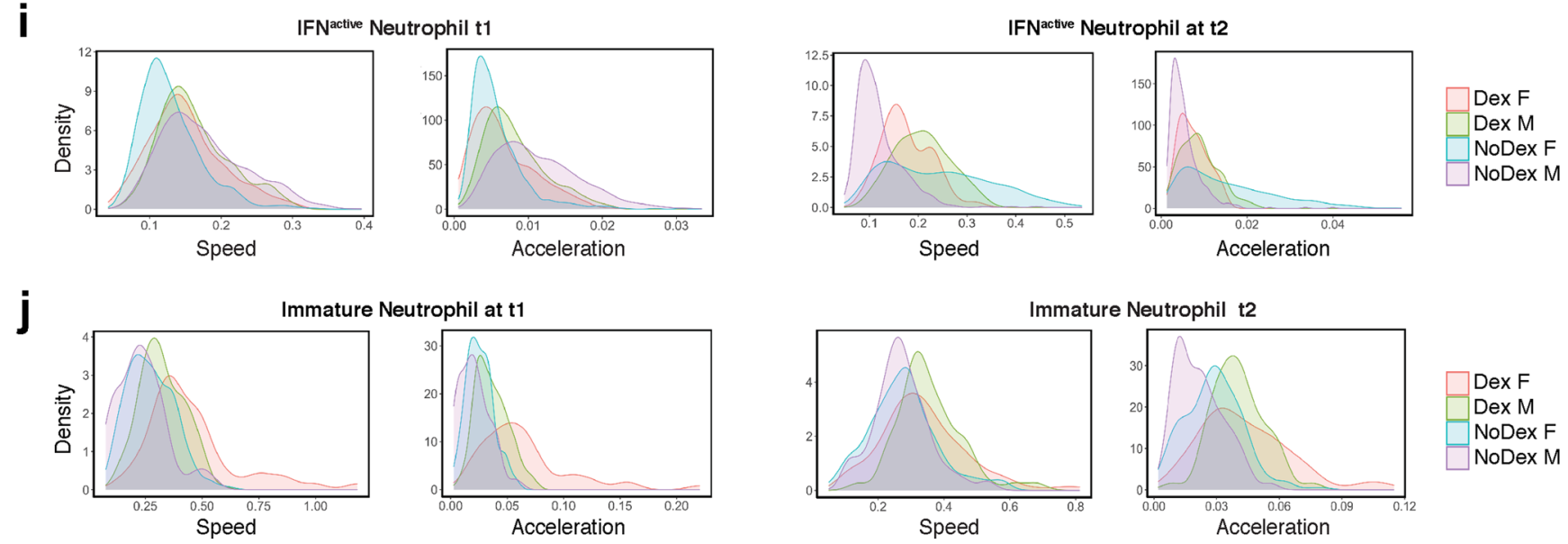

Extended Data Fig. 9 | See next page for caption. 
Extended Data Fig. 9 | Dexamethasone attenuates neutrophil response in a sexually dimorphic fashion. a-b. ICU mortality rates of sex-separated patients with (a) or without COVID-19 (b) comparing pre-dexamethasone (January 2020 till May 317, 2020) and post-dexamethasone (June $1^{\text {st }}$, 2020, till May $\left.31^{\text {st }}, 2021\right)$ standard of care time periods. c. Number of genes that are uniquely or jointly regulated with dexamethasone between males and females. d. Differential magnitude or direction of regulation within dexamethasone-induced DEGs jointly regulated by both sexes. e. Heatmap depicting dexamethasone-induced shifts in cellular composition at $\mathrm{t} 1$ and $\mathrm{t} 2$ and accompanying bar plots showing magnitude of divergence between male and female response. Dexamethasone-induced shifts in neutrophil state composition at $\mathrm{t} 1$ and $\mathrm{t} 2 \mathrm{along}$ with magnitude of divergence between male and female response. f. Module score of ISG signatures in ISG-active neutrophils across sex and dexamethasone treatment at $\mathrm{t} 1$ and $\mathrm{t} 2$. Statistical significance was assessed using an ANOVA test followed by bonferroni-corrected two-sided pair-wise t-tests. ${ }^{\star} p$-value $<0.05$; ${ }^{\star \star} p$-value $<0.01$; ${ }^{\star \star \star} p$-value $<$ 0.001; ns p-value > 0.05. Absolute p-adjusted values are provided in Supplementary Table 8. Center line indicates median data point. g. Comparison of proportion of neutrophils in whole blood samples from sex-separated cohorts. $\mathbf{h}$. Comparison of neutrophil composition across sex in dexamethasonetreated patients at 72 hours and 7 days post-ICU admission. i-j. Histograms depicting dynamo-calculated distribution of cell speed (length of velocity vectors) and acceleration (subspaces where velocity undergoes dramatic changes in magnitude or direction) of all IFN-active (i) and immature (j) neutrophils, separated by sex and dexamethasone treatment for both $\mathrm{t} 1 \mathrm{and} \mathrm{t} 2$. 

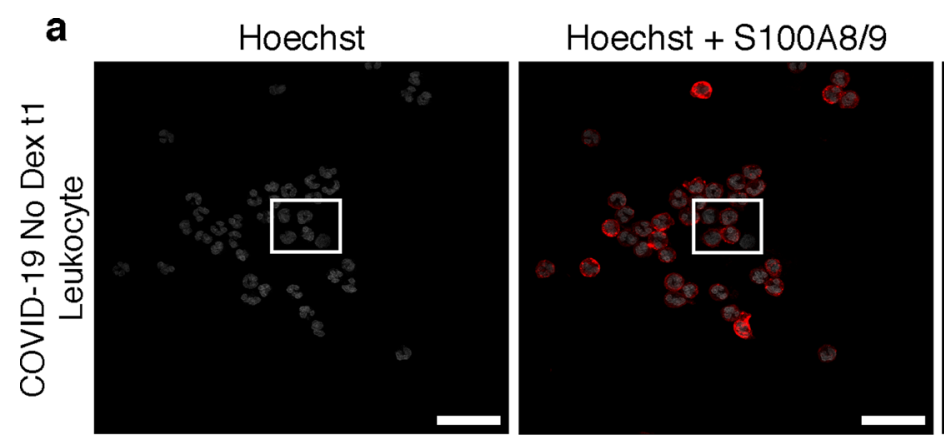

Hoechst + IFITM1
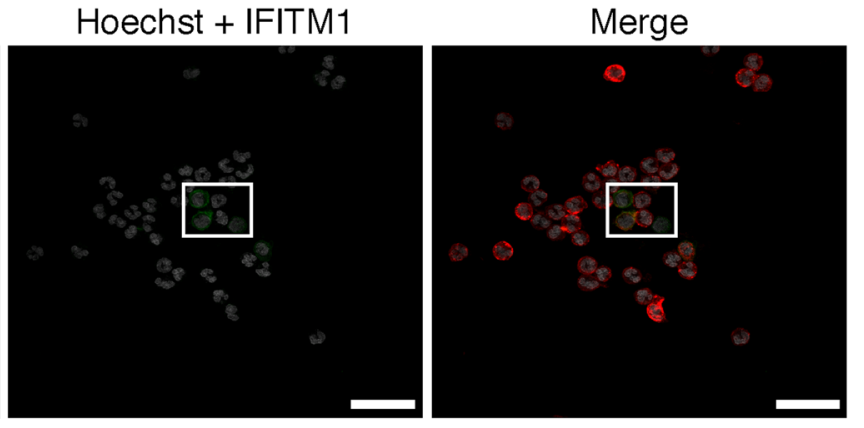

b

Hoechst

CD24

IFITM1

$\mathrm{S} 100 \mathrm{~A} 8 / 9$

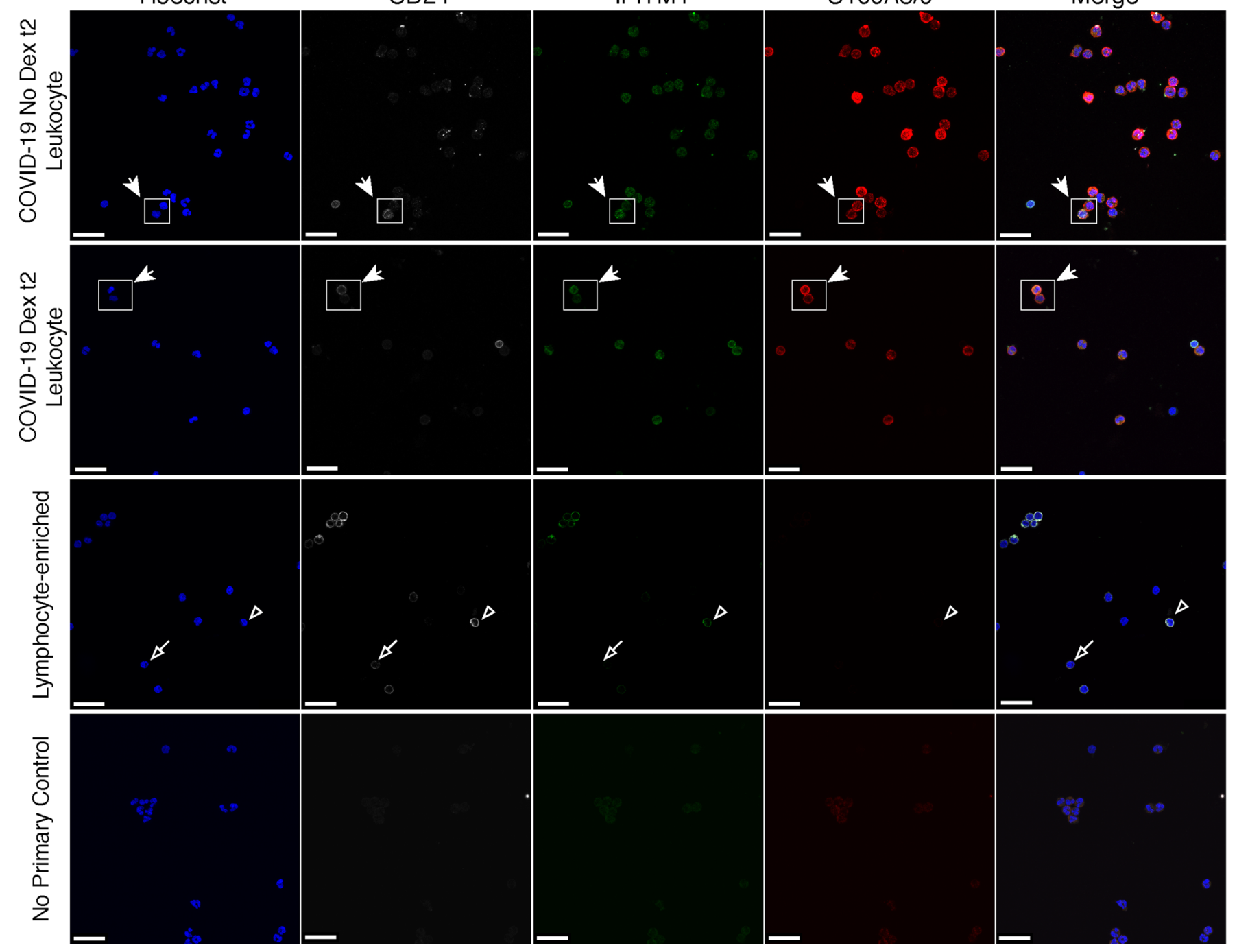

Merge

Extended Data Fig. 10 | Immunofluorescence staining for neutrophil population markers. Immunofluorescence representative images showing co-staining of Hoechst dye, anti-calprotectin (S100A8/A9), anti-IFITM1, and anti-CD24 antibodies on either leukocyte- or lymphocyte-enriched cytospin preparations from COVID-19+ve patients at t1 (a) or t2 (b). Rectangles highlight field of view shown in Fig. 1h (a) and Extended Data Fig. 6i (b). Representative images are shown; $n=3$ for each group; Scale bar, $25 \mu \mathrm{m}$. 


\section{Reporting Summary}

Nature Research wishes to improve the reproducibility of the work that we publish. This form provides structure for consistency and transparency in reporting. For further information on Nature Research policies, see our Editorial Policies and the Editorial Policy Checklist.

\section{Statistics}

For all statistical analyses, confirm that the following items are present in the figure legend, table legend, main text, or Methods section.

$\mathrm{n} / \mathrm{a}$ Confirmed

$\bigotimes$ The exact sample size $(n)$ for each experimental group/condition, given as a discrete number and unit of measurement

$\bigotimes$ A statement on whether measurements were taken from distinct samples or whether the same sample was measured repeatedly

The statistical test(s) used AND whether they are one- or two-sided

Only common tests should be described solely by name; describe more complex techniques in the Methods section.

A description of all covariates tested

A description of any assumptions or corrections, such as tests of normality and adjustment for multiple comparisons

A full description of the statistical parameters including central tendency (e.g. means) or other basic estimates (e.g. regression coefficient)

AND variation (e.g. standard deviation) or associated estimates of uncertainty (e.g. confidence intervals)

For null hypothesis testing, the test statistic (e.g. $F, t, r$ ) with confidence intervals, effect sizes, degrees of freedom and $P$ value noted Give $P$ values as exact values whenever suitable.

Х $\square$ For Bayesian analysis, information on the choice of priors and Markov chain Monte Carlo settings

Х $\square$ For hierarchical and complex designs, identification of the appropriate level for tests and full reporting of outcomes

$\triangle \square$ Estimates of effect sizes (e.g. Cohen's $d$, Pearson's $r$ ), indicating how they were calculated

\section{Our web collection on statistics for biologists contains articles on many of the points above.}

\section{Software and code}

Policy information about availability of computer code

Data collection Single-cell RNA-Seq library construction, alignment, and quality control: 10X Genomics NextGEM Gel Bead emulsions (Version 3.1). Illumina NovaSeq S2 and SP 100 cycle dual lane flow cells over multiple rounds to ensure each sample received approximately 32,000 reads per cell. MIlliPLEX cytokine/chemokine and a 14 MilliPLEX soluble cytokine arrays (Millipore Sigma, Oakville, ON, Canada) on a Luminex Model 200 Luminometer (Luminex Corporation, Austin, TX).

Shotgun proteomics was performed using Liquid Chromatography and Mass Spectrometry (LC-MS/MS) using FAIMS Pro (Thermo Fisher Scientific). Briefly, precipitated protein pellet was resuspended in 100 microL of $50 \mathrm{mM}$ triethylammonium bicarbonate (TEAB) buffer followed by tryptase digestion overnight at $37^{\circ} \mathrm{C}$. TMT-6plex ${ }^{\mathrm{TM}}$ Isobaric Labeling Reagents (90061, Thermo Fisher) were resuspended in anhydrous acetonitrile and added to each sampleand incubated at room temperature for $1 \mathrm{~h}$. The TMT labeling reaction was quenched by $2.5 \%$ hydroxylamine for $15 \mathrm{~min}$ at room temperature. TMT labeled samples were combined and acidified in $100 \%$ trifluoroacetic acid to $\mathrm{pH}<3.0$ and subjected to $\mathrm{C} 18$ chromatography (Sep-Pak) according to manufacturer recommendations. Samples were stored at $-80^{\circ} \mathrm{C}$ before lyophilization, followed by resuspension in $1 \%$ formic acid before liquid chromatography and tandem mass spectrometry analysis. Tryptic peptides were analyzed on an Orbitrap Fusion Lumos Tribrid mass spectrometer (Thermo Scientific) operated with Xcalibur (version 4.0.21.10) and coupled to a Thermo Scientific Easy-nLC (nanoflow liquid chromatography) 1200 System. Tryptic peptides were loaded onto a C18 trap (75microm x 2cm; Acclaim PepMap 100, P/N 164946; ThermoFisher). The fragment ions (MS2) were analyzed in the Orbitrap at a resolution of 15,000. The AGC and the maximum injection time were set at $1 \times 105$ and $105 \mathrm{~ms}$, respectively. The first mass for the MS2 was set at 100 to acquire the TMT reporter ions. Dynamic exclusion was enabled for 45 seconds to avoid of the acquisition of same precursor ion having a similar $\mathrm{m} / \mathrm{z}$ (plus or minus 10ppm).

Imaging was done using a VS-120 slide scanner (Olympus) and high resolution image imaging was done using an SP8 spectral confocal microscope (Leica). Image processing was completed in Fiji (V.2.1.0). 
Single-cell RNA-Seq computational analyses and workflows: R package Seurat (v.3.9, v.4) for normalization, scaling, integration, multi-modal reference mapping, louvain clustering, dimensionality reduction, differential expression analysis, and visualization. Fitting was done with Laplace approximation using the 'glmer' function in the 'Ime4' R package (v.1.1-27.1) and p-values were calculated using the R package 'car' (v.3.0-11). Differential cell-cell interaction networks were reconstructed using CellChat v1.0.0.

Consensus DEGs and perturbation scores: Differentially expressed genes (DEGs) were those with an average log fold change (FC) greater than 0.25 ( $p$-adjusted < 0.05) as determined by Seurat's Wilcoxon rank-sum test. Consensus stacked bars were generated using constructConsensus function for genes exhibiting reproducible changes across patients ( $>3$ for 72 -hour comparisons, $>2$ for 7-day comparisons). Gene Set Enrichment analyses were performed using gProfiler's g:GOSt ( $p$-value cutoff $<0.05$ ). Perturbation scores were visualized using Nebulosa v.1.0.2.

Constructing cellular trajectories using RNA velocity: RNA velocity command-line tool using the run10x command and human (GRCh38) annotations. Files read into Seurat (v.3.9, v.4) using the ReadVelocity function in SeuratWrappers v.0.2.0, normalized using SCTransform v.0.3.2, reduced and projected onto a UMAP, and exported as a .h5 file using the SaveH5Seurat function. RNA velocities were estimated using stochastic and dynamical models. Since both models yielded comparable results, stochastic model was used as default for all subsequent analyses.

Gene Regulatory Network reconstruction: Single-cell regulatory network inference and clustering (SCENIC) was employed to infer regulatory interactions between transcription factors (TFs) and their targetome by calculating and pruning co-expression modules. SCENIC repository is available at: https://github.com/aertslab/SCENIC. Targetome of TFs predicted as drivers of neutrophil states (stored in

'2.6_regulons_asGeneSet.Rds') was profiled using g:Profiler's functional enrichment analysis and genes intersecting with the Interferon pathway were plotted using iRegulon (Cytoscape plugin).

Epidemiological analysis: We used the Alberta provincial eCRITICAL oracle-based analytics database (Tracer) to query and extract Alberta COVID-19 ICU cases and volumes for this study. Aggregate data from sixteen individual adult ICUs was obtained over the study periods. The administration of dexamethasone was not possible to capture at an aggregate level; therefore, we queried the database for patients admitted to ICU prior to dexamethasone becoming standard of care in our Province (pre-dexamethasone era; January 2020 till May 31st, 2020) versus dexamethasone as standard of care for severe COVID-19 (June 1st, 2020, till May 31st, 2021).

Shotgun proteomics using Liquid Chromatography and Mass Spectrometry (LC-MS/MS): Samples were subjected to a quantitative proteomics workflow as per supplier (Thermo Fisher) recommendations using tryptase digestion (5microgram trypsin per 100microgram of protein).

COVID Neutrophil Atlas: RShiny v1.1.0, shinyLP v.1.1.2, and shinythemes v.1.1.2 packages.

Full data analysis is described in the Methods section.

For manuscripts utilizing custom algorithms or software that are central to the research but not yet described in published literature, software must be made available to editors and

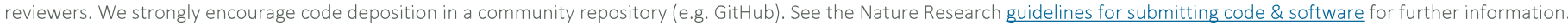

\section{Data}

Policy information about availability of data

All manuscripts must include a data availability statement. This statement should provide the following information, where applicable:

- Accession codes, unique identifiers, or web links for publicly available datasets

- A list of figures that have associated raw data

- A description of any restrictions on data availability

eCRITICAL is a secure patient database that is not publicly accessible. Requests for access to patient related data, either de-identified, summary or patient level data must be approved by eCRITICAL with an appropriate ethics protocol and may require approval by Alberta Health Services. Single cell RNA-Seq datasets are available at NCBI GEO (which automatically makes SRA deposit) at the following accession: GSE157789. Single-cell datasets can be further explored on our companion portal at http://biernaskielab.ca/COVID_neutrophil or http://biernaskielab.com/COVID_neutrophil. Velocyto-generated LOOM files and processed R objects are available for reanalysis from: http://doi.org/10.6084/m9.figshare.14330795. Whole blood bulk RNA-Seq datasets employed as an independent validation cohort were downloaded from GSE157103. BALF scRNA-Seq datasets from severe and moderate COVID-19 were downloaded from GSE145926. Processed BALF scRNA-Seq objects from patients with bacterial pneumonia and COVID-19 (archived at GSE167118) were downloaded from authors' archive: https://figshare.com/articles/ dataset/_13608734. Mass spectrometry datasets are available via ProteomeXchange Consortium via the PRIDE partner repository with identifier PXD028429.

\section{Field-specific reporting}

Please select the one below that is the best fit for your research. If you are not sure, read the appropriate sections before making your selection.

$\bigotimes$ Life sciences Behavioural \& social sciences Ecological, evolutionary \& environmental sciences

For a reference copy of the document with all sections, see nature.com/documents/nr-reporting-summary-flat.pdf 


\section{Life sciences study design}

All studies must disclose on these points even when the disclosure is negative.

Sample size

Sample size for the non-Dexamethasone-treated patients was restricted due to Dexamethasone becoming standard of care worldwide for ICU patients. Number of dexamethasone-treated samples was pragmatically determined to roughly match their non-Dexamethasone counterpart. The unbiased nature of our interrogation precluded a priori sample size estimation as expected effect size could not be approximated.

Aggregated non-dexamethasone-treated COVID-19 $(n=12$ samples) and bacterial ARDS ( $n=9$ samples) recovered 1,872,659 cells that were sequenced to 38,410 post-normalization reads per cell. Likewise, aggregated COVID-19 samples with ( $n=9$ samples) or without ( $n=12$ samples) dexamethasone recovered 1,748,551 single cells sequenced to 51,415 post-normalization reads per cell. Aggregated healthy samples recovered 19,816 cells, including 1,912 post-QC neutrophils ( $n=5$ donors).

Data exclusions We excluded 1 sample (patient ID: C2) from scRNA-Seq analyses as this was collected from a donor who suffered a stroke and tested positive for COVID-19. The patient did not have respiratory issues and spent only 1 night in ICU before being transfered to the stroke ward. The decision to exclude was based strictly on a-priori clinical characteristics. To be comprehensive, we have uploaded this sample to GEO (GSM4775024) and have made a note that this donor was excluded from downstream analyses.

Replication Sequencing of patient samples happened between April 1st 2020 - Oct 30th 2020. As a result, multiple technical batches were generated by different individuals. Our analyses are based on merging these datasets using an MNN-based batch correction to avoid potential technical biases. Immunohistochemistry experiments were successfully replicated across two separate batches.

Differential gene expression analyses are presented as consensus stacked bars showing cumulative log fold changes (colored by individual sample contributions) that were generated using constructConsensus function for genes exhibiting reproducible changes across patients ( $>3$ for 72-hour comparisons, > 2 for 7-day comparisons).

Randomization Allocating patients into experimental groups (i.e. COVID-19 vs Bacterial ARDS) was not feasible.

Blinding Patient samples were obtained during the first wave of COVID-19 patients in Calgary, Alberta, Canada. All analyses were performed by personnel who were blinded to the patient status.

\section{Reporting for specific materials, systems and methods}

We require information from authors about some types of materials, experimental systems and methods used in many studies. Here, indicate whether each material, system or method listed is relevant to your study. If you are not sure if a list item applies to your research, read the appropriate section before selecting a response.

Materials \& experimental systems

Methods

n/a Involved in the study

$\square$ \Antibodies

Х Eukaryotic cell lines

\ $\square$ Palaeontology and archaeology $\mathrm{n} / \mathrm{a}$ Involved in the study

X $\square$ chIP-seq

Х Flow cytometry

\ $\square$ Animals and other organisms

Х| MRI-based neuroimaging

$\square$ Xuman research participants

Х Clinical data

$\bigotimes \square$ Dual use research of concern

\section{Antibodies}

Antibodies used

Isolated leukocyte and lymphocyte samples were fixed in $4 \%$ paraformaldahyde in PBS $(0.2 \mathrm{mM}$ and pH7.4), and spun in a cytocentrifuge ( $8 \mathrm{~min}$ at $300 \mathrm{~g}$ ) onto coated slides. Pathological lung sections (FFPE fixed and sectioned at $5 \mathrm{um}$ ) were deparaffinized in Slide Brite (Fisher Scientific NC968653) and rehydrated. Slides were permeabilized and blocked with $10 \%$ normal donkey serum in PBS (with $0.5 \%$ triton X-100), primary antibodies (full list presented below) were incubated at $40 \mathrm{C}$ overnight, followed by incubation with secondary antibodies (full list presented below) for $1 \mathrm{~h}$ at room temperature (RT). Cytospun slides were sequentially stained with CD24 (Abcam ab202073) on the same slides for 1h at RT, followed by donkey anti-rabbit-Alexa647 (Invitrogen A31573).

Primary antibodies:

S100A8/9 (Abcam ab22506), 1:250

IFITM1 (Abcam ab233545), 1:250

CD24 (Abcam ab202073), 1:250

Secondary antibodies:

donkey anti-rabbit-Alexa488 (Invitrogen A32790), 1:500

donkey anti-mouse-Alexa555 (Invitrogen A31570), 1:500

donkey anti-rabbit-Alexa647 (Invitrogen A31573), 1:500 


\section{Human research participants}

Policy information about studies involving human research participants

Population characteristics

Study inclusion required a minimal age of 18 , the ability to provide consent, or for most participants, the ability of a surrogate decision maker to provide regained capacity consent. All participants required an arterial catheter for blood draws, but the insertion of this catheter was at the discretion of the attending medical team. Participants required a positive clinical RNA COVID-19 test prior to enrollment, and evidence of bilateral lung infiltrates and hypoxemia consistent with ARDS. At the time of sample collections, all COVID-19+ enrolled individuals were culture negative for concurrent bacterial infections in the blood, urine, and sputum. The bacterial ARDS cohort required a negative COVID-19 test and a definitive microbiological diagnosis of bacterial pneumonia with chest imaging consistent with a diagnosis of ARDS. Patients were excluded from our study if they: 1. were on immunosuppressive therapies, 2. had established autoimmune disease, or 3 . had active malignancy. Since tocilizumab or other immunomodulatory agents were not approved for use in patients with severe COVID-19 in Alberta over the timespan of this study, none of them received these medications. While bacterial sepsis patients received appropriate antibiotic treatments, none were prescribed immunosuppressive or steroid therapy. All bacterial sepsis patients had lung infections caused by gram-positive cocci (4 Staphylococcus aureus and 2 Streptococcus pneumoniae). Participants were required to have a definitive diagnosis and appropriate consent and samples collected within $72 \mathrm{hrs}$ of admission to the ICU in order to be included. Timepoint 1 (T1) refers to the first blood draw, while T2 was a repeat blood draw taken 7 days after $\mathrm{T} 1$, if the participant remained in the $\mathrm{ICU}$, and had an arterial catheter. For each participant, whole blood was collected via the arterial catheter and immediately processed for analysis. Healthy blood donors were recruited by university-wide advertisement and required that participants were: 1 . not on immunomodulatory medications, 2 . were asymptomatic for SARS-CoV-2, 3. did not receive vaccination against SARS-CoV-2, and 4. did not have underlying immune disorders.

Our study recruited 6 Bacterial ARDS (3 Males, 3 Females), 8 non-dexamethasone-treated COVID-19 (5 Males, 3 Females), 6 dexamethasone-treated COVID-19 (4 Males, 2 Females), and 5 Healthy (3 Males, 2 Females) donors. Age, ethnicity and clinical characteristics of enrolled individuals is provided in our Supplementary Table 2.

Recruitment

All patients were enrolled following admission to any of the four adult intensive care units at South Health Campus, Rockyview General Hospital, Foothills Medical Center or Peter Lougheed Center in Calgary, Alberta, Canada. These units serve the entire city representing all communities and diverse groups. Patient admission to the ICU was determined by the attending ICU physician based on the need for life sustaining interventions, monitoring and life-support. The research teams did not participate in clinical decisions, including diagnosis and treatment.

Potential recruitment biases include: patients without a surrogate decision maker could not be consented for enrollment and catchment area was limited to hospitals in Calgary, Alberta. As a result, participants without surrogate or family may be under represented or absent in our analysis, however it is not clear how this factor associates with underlying medical conditions, socioeconomic factors, ethnicity, sex or gender.

Ethics oversight

This study was approved by the Conjoint Health Research Ethics Board (CHREB) at the University of Calgary (Ethics ID: REB20-0481) and is consistent with and the Declaration of Helsinki. 